Check for updates

Cite this: RSC Adv., 2019, 9, 6804

Received 13th December 2018 Accepted 18th February 2019

DOI: 10.1039/c8ra10247c

rsc.li/rsc-advances

\section{Metal-mediated synthesis of pyrrolines}

\author{
Noelia S. Medran, Agustina La-Venia and Sebastian A. Testero (iD *
}

The five-membered, nitrogen-containing pyrroline ring is a privileged structure. This ring is present in many bioactive compounds from natural sources. Pyrrolines-the dihydro derivatives of pyrroles-have three structural isomer classes, depending on the location of the double bond: 1-pyrrolines (3,4-dihydro- $2 \mathrm{H}$ pyrroles), 2-pyrrolines (2,3-dihydro- $1 \mathrm{H}$-pyrroles) and 3-pyrrolines (2,5-dihydro- $1 \mathrm{H}$-pyrroles). This review aims to describe the latest advances for the synthesis of pyrrolines by transition metal-catalyzed cyclizations. Only reactions in which the pyrroline ring is formed by metal promotion are described. Transformations of the pyrroline ring in other heterocycles, and the structural manipulations of the pyrroline itself are not discussed. The review is organized into three parts, each covering the metalmediated synthesis of the three pyrroline isomers. Each part is subdivided according to the metal involved, and concludes with a brief description of notable biological activities within the class.

\section{Introduction}

Heterocyclic ring systems are the fundamental building blocks in the vast majority of drugs used to treat animal and human diseases. Among these heterocyclic rings, those containing nitrogen are the most significant. Pyrrolines-the dihydro

Instituto de Química Rosario - IQUIR (CONICET), Facultad de Ciencias Bioquímicas y Farmacéuticas, Universidad Nacional de Rosario, Suipacha 531, Rosario, S2002LRK, Argentina. E-mail: testero@iquir-conicet.gov.ar; Web: http://www.iquir-conicet.gov. ar/eng/

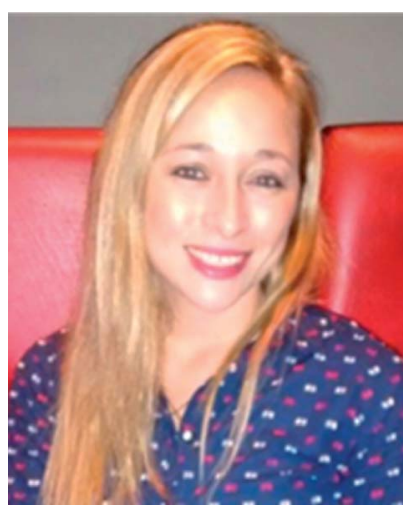

Noelia S. Medran completed her PhD in synthetic organic chemistry at the Instituto de Quimica Rosario in 2017, under the supervision of Prof. Sebastian A. Testero. Since 2017 she is carrying out a postdoctoral research in organoboron chemistry and computational chemistry, particularly applied to Diels-Alder reactions, with Prof. Silvina C. Pellegrinet at the same institute. Since 2015 she is teacher assistant at the department of Medicinal Chemistry of National Argentinean University (UNR). Her research interests center on medicinal chemistry, organometallic chemistry, organoboron chemistry, and computational chemistry. derivatives of pyrroles-have received considerable attention lately since they exhibit a variety of biological activities. Pyrrolines have three structural isomer classes (Fig. 1), depending on the location of the double bond: 1-pyrrolines (3,4-dihydro- $2 \mathrm{H}^{-}$ pyrroles), 2-pyrrolines (2,3-dihydro- $1 H$-pyrroles) and 3-pyrrolines (2,5-dihydro- $1 H$-pyrroles).

Pyrrolines are considered privileged structures as reflected by their presence in many bioactive compounds from natural sources $^{1-9}$ such as hemes, ${ }^{10}$ chlorophyll, ${ }^{10}$ and alkaloids; ${ }^{11,12}$ as well as in bioactive synthetic molecules. ${ }^{13-19}$

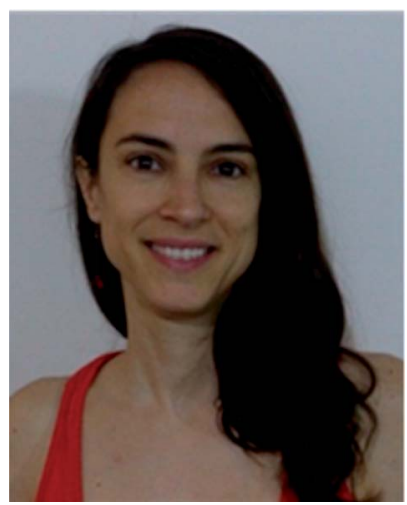

Agustina La-Venia completed her PhD in synthetic organic chemistry at the Instituto de Química Rosario (IQUIR) in 2011, under the supervision of Prof. Dr Mirta P. Mischne and Prof. Dr Ernesto G. Mata. Between 2011 and 2015, she carried out a postdoctoral research in the combinatorial synthesis of constrained peptidomimetics at the Palacky University (UPOL), Czech Republic under Prof. Dr Viktor Krchňák. Since 2015, she is Junior Researcher of the Argentine National Council of Research at IQUIR. She has been teacher assistant at the department of Organic Chemistry of National Argentinean University (UNR) between 2005 and 2011, and since 2015 she is teaching at the department of Medicinal Chemistry of UNR. Her research interests center on medicinal chemistry, combinatorial chemistry, solid phase synthesis, metal catalysis, and peptidomimetics. 


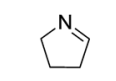

1-pyrrolines

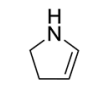

2-pyrrolines

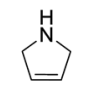

3-pyrrolines
Fig. 1 Structural isomers of the pyrrolines.

1-Pyrrolines are cyclic imines whose reactivity allows synthetic manipulation through nucleophilic attack on the prochiral endocyclic imine. Accordingly, stereoselective transformations can occur. ${ }^{20}$ 2-Pyrrolines possess an enamine moiety that allows the further functionalization of the ring system. 2Pyrrolines are found frequently in the literature under the name "2,3-dihydropyrroles" since the monohydrogenation of pyrroles leads directly to 2,3-dihydropyrroles. In contrast, the cyclic amine and alkene functional groups of the 3-pyrrolines react separately. When this cyclic core is used as precursor, further modifications often involve the double bond, which can be easily transformed e.g. by hydrogenation, (di)halogenation, and dihydroxylation. Thus the 1-, 2- and 3-pyrrolines represent appealing intermediates to obtain pyrroles and pyrrolidines through oxidation ${ }^{21}$ and reduction, ${ }^{22,23}$ respectively. Due to the remarkable breadth of their reactivity, pyrrolines are useful intermediates in the preparation of more complex heterocycles. ${ }^{24-37}$

A variety of well-established methods of pyrrolines synthesis are available. ${ }^{38,39}$ These methods include intramolecular cyclizations of bifunctional compounds and multi-component cyclizations, $^{39-45}$ 1,3-dipolar cycloadditions, ${ }^{46-51}$ photo- and thermoinduced reactions, ${ }^{52-54}$ and ring expansion of aziridines, ${ }^{55,56}$ among others. However, metal-mediated syntheses have emerged as a valuable complement to these methods ${ }^{20,57,58}$ due to their high atom economy, their mild reaction conditions, and the high functional group tolerance of the transition metalcatalyzed reactions. This review discusses the latest advances $^{24,38,39,59}$ (from 2011 to December 2018) for the synthesis of pyrrolines by transition metal-catalyzed cyclizations. Only

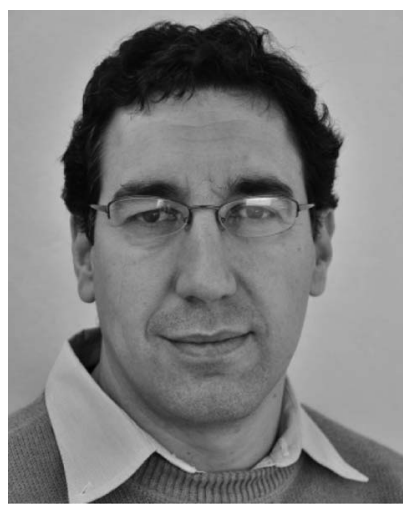

Sebastian A. Testero completed his PhD in synthetic organic chemistry at the Instituto de Química Rosario in 2005, under the supervision of Prof. $R$. A. Spanevello. Then he carried out a postdoctoral research in solidphase synthesis with Prof. E. G. Mata at the same institute. Between 2007 and 2011, he was a postdoctoral research associate at the University of Notre Dame at the Mobashery lab working in medicinal chemistry. Since 2015, he is Adjunct professor of Organic Chemistry and from 2017 an independent researcher of the Argentine National Council of Research. His research interests center on medicinal chemistry, combinatorial chemistry, ozonolysis and organometallic chemistry including metathesis and gold catalysis. reactions in which the pyrroline ring is formed by metal promotion are described. Transformations of the pyrroline ring in other heterocycles, and the structural manipulations of the pyrroline itself are not discussed. The review is organized into three parts, each covering the metal-mediated synthesis of the three pyrroline isomers. Each part is subdivided according to the metal involved, and concludes with a brief description of notable biological activities within the class and a synthetic sequence which involve a metal-mediated synthesis of pyrrolines as intermediate towards a more complex heterocycle.

\section{Synthesis of pyrrolines}

\subsection{Synthesis of 1-pyrrolines}

The 1-pyrroline core is exemplified by numerous compounds with biological activity (Fig. 2). Examples include the iminosugar nectrisine (1), ${ }^{60}$ discovered as an immunomodulator; the iminosaccharide 2 that has glycosidase inhibitory activity; ${ }^{\mathbf{1 5 , 6 1}}$ and the 1-pyrroline 3 that has antihypertensive properties. ${ }^{\mathbf{1 4}} \beta$ Trifluoromethylated 1-pyrrolines (4-6) ${ }^{62}$ are nitric oxide synthase inhibitors ${ }^{63}$ and as such possess anti-infective, ${ }^{64-66}$ antitumor, ${ }^{67}$ and anti-inflammatory activities. ${ }^{65,68}$ The steroidal alkaloid plakinamine A (7) shows antimicrobial activity against

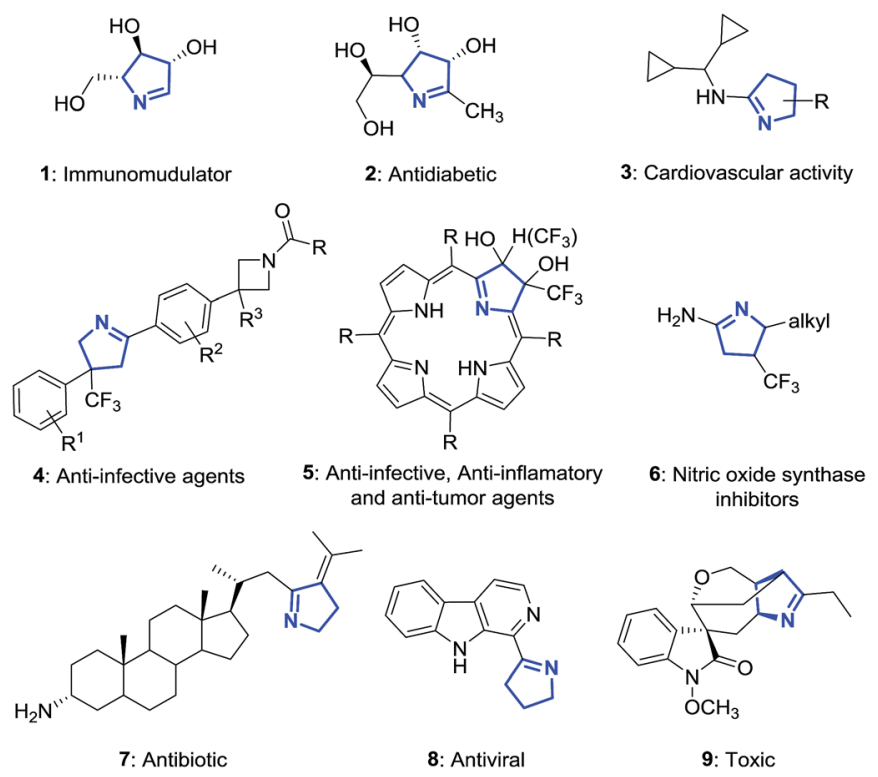

Fig. 2 Selected examples of biologically active 1-pyrrolines.

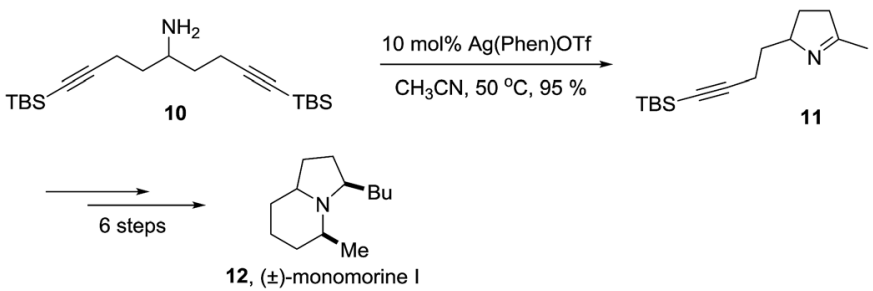

Scheme 1 Synthesis of the alkaloid $( \pm)$-monomorine I through the 1pyrroline 11 . 


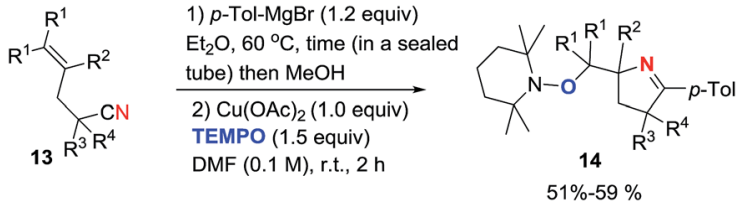

Scheme $2 \mathrm{Cu}(I)$-mediated intramolecular aminooxygenation of alkenylimines towards 1-pyrrolines.
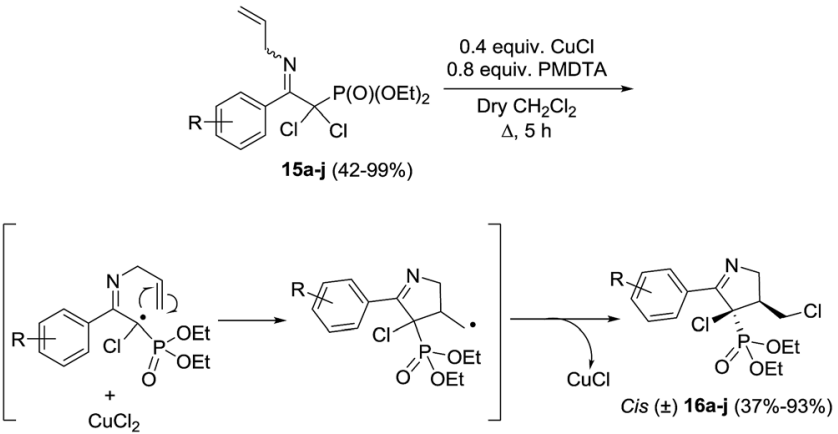

Scheme 3 Copper-catalyzed heteroatom transfer radical cyclization (HATRC) towards 1-pyrrolines.

S. aureus and C. albicans, ${ }^{69}$ whereas eudistomin (8) has antiviral activity. $^{2}$ The alkaloid gelsenicine (9) is recognized for its high toxicity. $^{70}$

A selected example of 1-pyrroline used as an intermediate towards a more complex heterocycle is described in the Scheme 1. Helquist et al. reported a silver-catalyzed hydroamination of the aminoalkyne $\mathbf{1 0}$ that led to 1-pyrroline $\mathbf{1 1}$ which was applied to a seven step synthesis of the alkaloid $( \pm)$-monomorine I in $26 \%$ overall yield. ${ }^{27}$

2.1.1. Synthesis of 1-pyrrolines by copper catalysis. Chiba et $a .^{71}$ developed a method for the synthesis of oxymethylsubstituted pyrrolines employing $\mathrm{Cu}(\mathrm{OAc})_{2}$-mediated intramolecular aminooxygenation of alkenylimines with TEMPO (Scheme 2). The addition of a Grignard reagent (such as $p$-tolylmagnesium bromide) to a range of alkenyl carbonitriles $\mathbf{1 3}$ was performed in a sealed tube at $60{ }^{\circ} \mathrm{C}$. $\mathrm{MeOH}$ was used to protonate the products, and DMF was added to reach a concentration of $0.1 \mathrm{M}$. Immediately, 1 equivalent of $\mathrm{Cu}(\mathrm{OAc})_{2}$ and 1.5 equivalents of TEMPO were added. The aminooxygenation proceeded smoothly at room temperature affording (after $2 \mathrm{~h}$ ) diverse oxymethyl pyrrolines $\mathbf{1 4}$ in moderate yields (typically 50\%). Various other Grignard reagents were equally successful.

Stevens et al. ${ }^{72}$ synthesized a library of ten 1-pyrrolines $\mathbf{1 6 a}-\mathbf{j}$ from the $\alpha, \alpha$-dichlorinated imines $\mathbf{1 5 a}-\mathbf{j}$ using a heteroatom transfer radical cyclization (HATRC) (Scheme 3). The freeradical ring closure reaction was performed with $\mathrm{CuCl}$ in presence of $N, N, N^{\prime}, N^{\prime \prime}, N^{\prime \prime}$-pentamethyldiethylenetriamine (PMDTA) as a ligand. Other ligands such as $N, N, N^{\prime}, N^{\prime}$-tetramethylethylenediamine (TMEDA) proved equally efficient. The addition of the ligands modifies the solubility and the redox potential of the copper catalyst, thus improving its activity. Formation of the five-membered ring proceeds through a radical 5-exo-trig

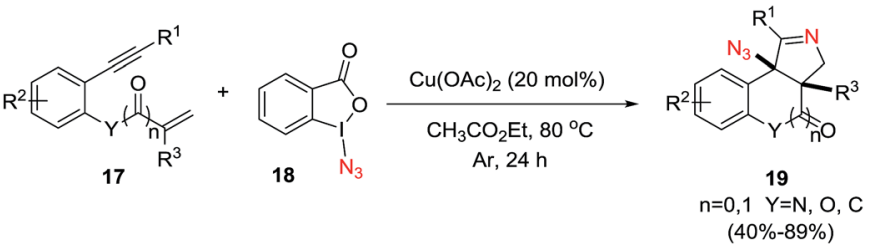

Scheme $4[2+2+1]$ Annulation/azidation of $1, n$-enynes as an entry to fused 1-pyrrolines.

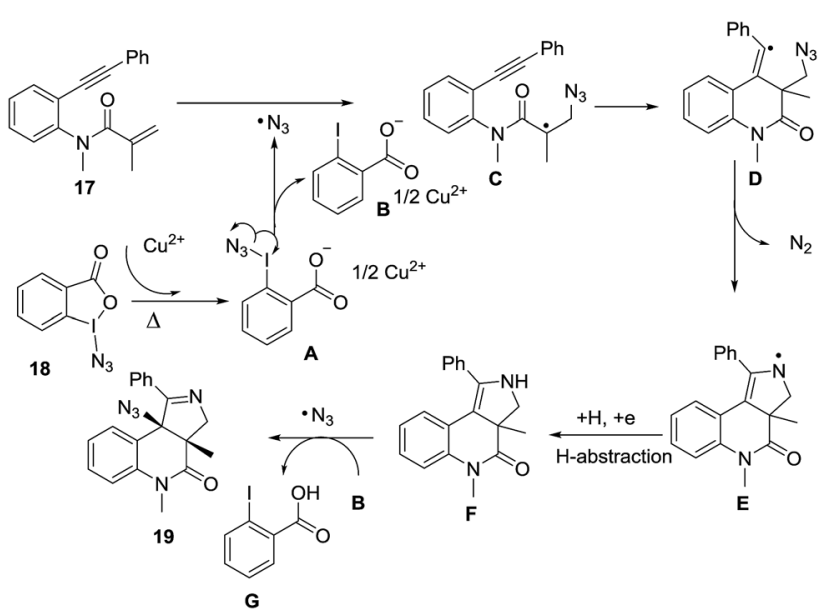

Scheme 5 Proposed mechanism of $[2+2+1]$ annulation/azidation of 1,n-enynes.

cyclization. Two stereogenic centers are generated during the ring closure. The reaction displays an excellent cis/trans diastereoselectivity (diastereoisomeric ratios are all in the range of 90/10). The authors attribute this diastereoselectivity to the steric hindrance caused by the ethoxy substituents of the phosphonate.

Li et $a l .{ }^{73}$ developed a novel selective copper-catalyzed, azide radical-mediated, $[2+2+1]$ annulation of benzene-linked 1, $n$ enynes $(n=6,7)$ to give fused pyrrolines 19 (Scheme 4). Azidobenziodoxolone $\mathbf{1 8}$ was the source of the azide radical. Other azide reagents such as $\mathrm{TMSN}_{3}$ and $\mathrm{NaN}_{3}$ failed to produce the fused pyrrolines. This one-step synthesis of fused pyrrolines proceeds via the generation of the azide radical from azidobenziodoxolone 18 with the aid of the $\mathrm{Cu}^{2+}$ species as catalyst (Scheme 5). Addition of the azide radical to the alkene moiety of enyne $\mathbf{1 7}$ affords an alkyl radical intermediate which undergoes intramolecular addition to the alkyne moiety to give fused pyrroline $\mathbf{F}$ as an intermediate. Finally, a second azide radical is incorporated to furnish the final fused pyrroline structures. Internal alkenes with $\mathrm{R}_{1} \neq$ aliphatic are suitable substrates.

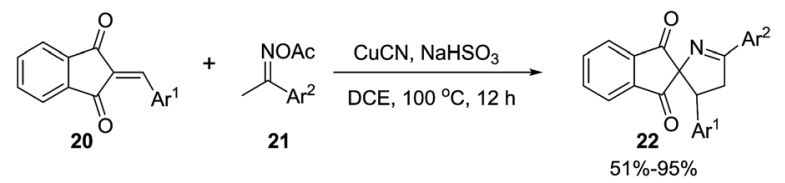

Scheme 6 Copper-catalyzed heteroannulation reaction between aryl ketone-derived ketoxime acetates and 2-arylideneindane-1,3-dione. 


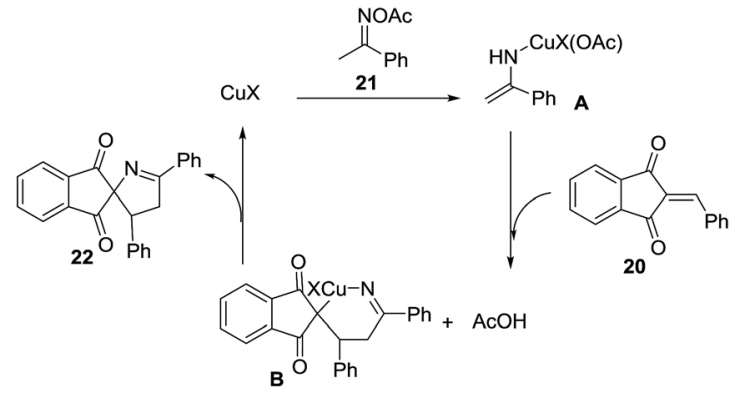

Scheme 7 Proposed mechanism for the copper-catalyzed heteroannulation reaction between ketoxime acetates and 2-arylideneindane-1,3-dione.

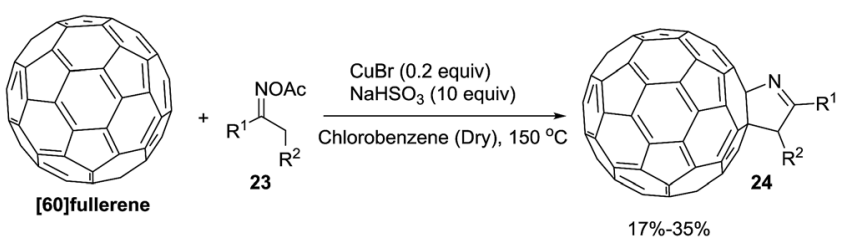

Scheme 8 Copper(I)-catalyzed heteroannulation of [60]fullerene with ketoxime acetates.

Li et $a l .{ }^{74}$ developed an efficient copper-catalyzed heteroannulation reaction between 2-arylideneindane-1,3-diones (20) and ketoxime acetates (21) for the straightforward synthesis of spiro[indane-1,3-dione-1-pyrrolines] 22 (Scheme 6). The methodology shows broad substrate scope and tolerates a wide range of functionalities in both the 2-arylideneindane-1,3-diones and aromatic ketoxime acetates. Alkyl ketoxime acetates failed to deliver the spiro compounds.

The proposed mechanism for this transformation begins with the cleavage of the $\mathrm{N}-\mathrm{O}$ bond in the ketoxime acetate 21 by

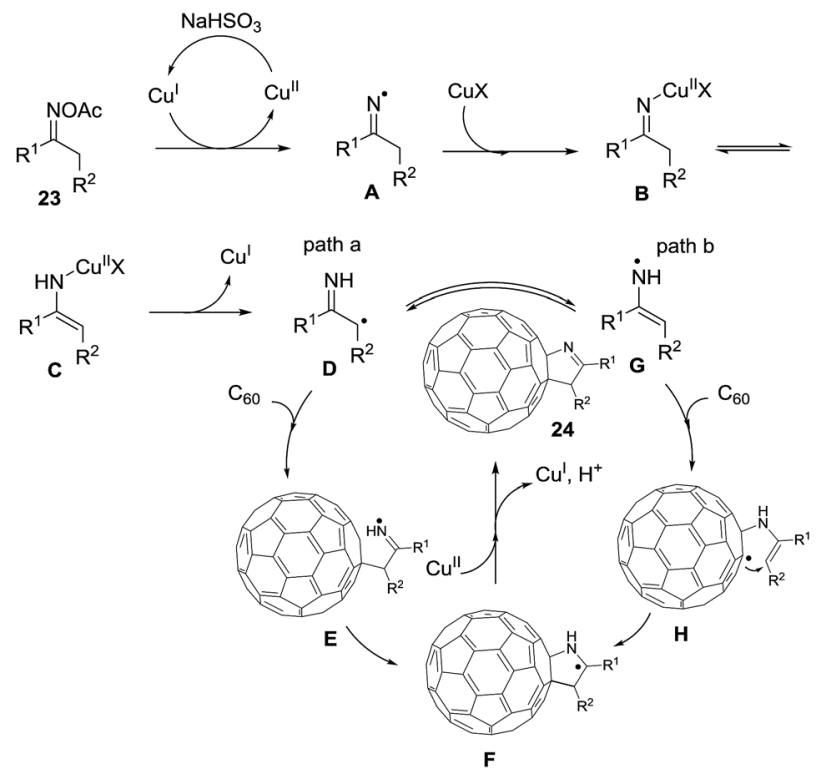

Scheme 9 Proposed mechanism of the heteroannulation of [60] fullerene with ketoxime acetates.

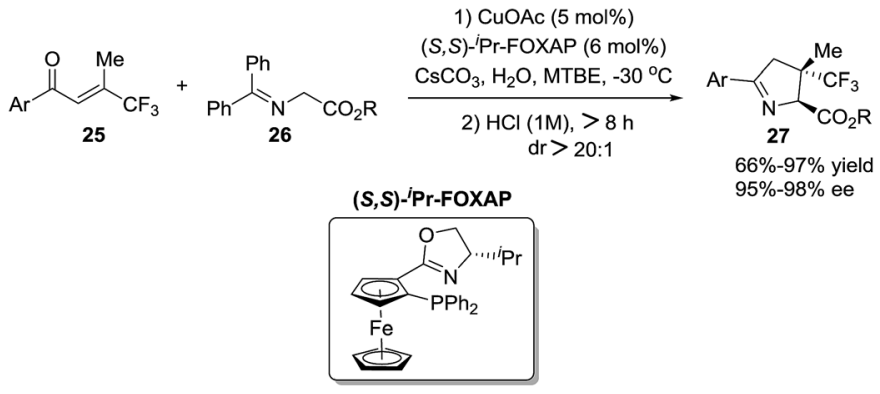

Scheme $10 \mathrm{Cu}(\mathrm{I})$-catalyzed Michael addition of ketiminoesters to $\beta$ trifluoromethyl $\beta, \beta$-disubstituted enones.

cuprous cyanide, through an oxidative addition (Scheme 7). As a result, the copper enamide $\mathbf{A}$ intermediate is formed, which reacts with the 2-arylideneindane-1,3-diones $\mathbf{2 0}$ to give intermediate $\mathbf{B}$. The product $\mathbf{2 2}$ is formed, and the catalyst regenerated, by an intramolecular redox heteroannulation of $\mathbf{B}$.

Wang et al. ${ }^{75}$ constructed novel 1-fulleropyrrolines 24 using a cuprous bromide-catalyzed heteroannulation reaction of [60] fullerene with ketoxime acetates $\mathbf{2 3}$ (Scheme 8). The proposed mechanism begins with ketoxime $\mathrm{N}-\mathrm{O}$ cleavage by $\mathrm{Cu}(\mathrm{I})$ to give an imino radical with formation of the $\mathrm{C}-\mathrm{C}$ and $\mathrm{C}-\mathrm{N}$ bonds (Scheme 9).

Zhang et al. ${ }^{76}$ reported a straightforward one-pot synthesis of 1-pyrrolines bearing two contiguous stereocenters exemplified by structure 27 , where one is a trifluoromethyl-substituted quaternary carbon (Scheme 10). The copper-catalytic process

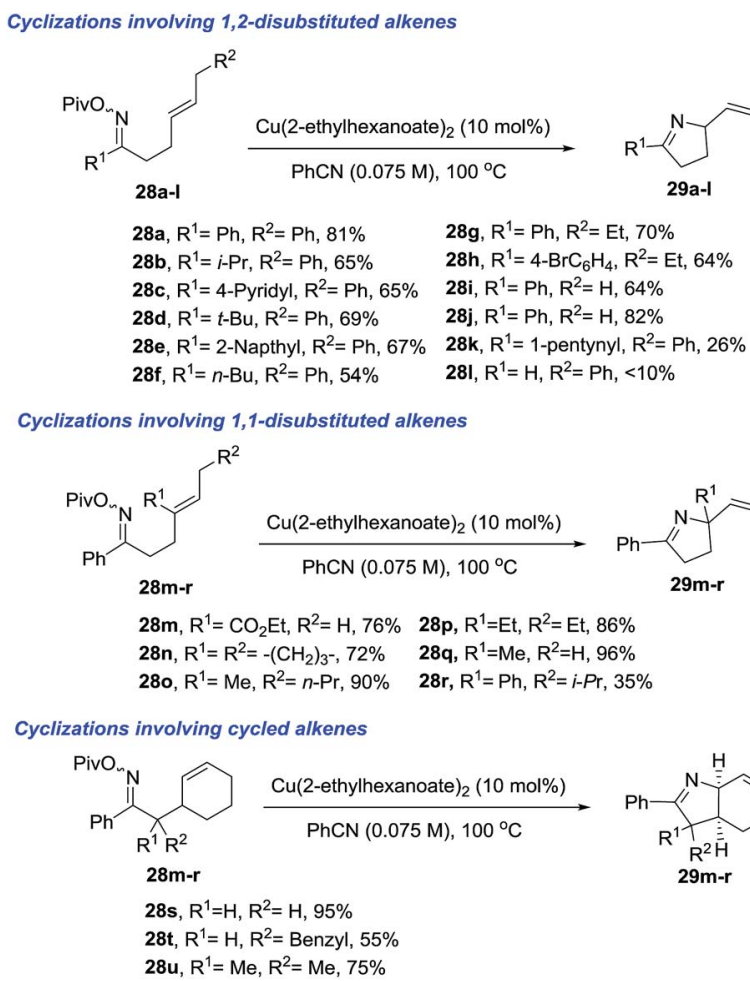
28e, $R^{1}=2-$ Napthyl, $R^{2}=P h, 67 \% \quad$ 28k, $R^{1}=1$-pentynyl, $R^{2}=P h, 26 \%$

28m, $R^{1}=\mathrm{CO}_{2} E t, R^{2}=\mathrm{H}, 76 \% \quad \mathbf{2 8 p}, R^{1}=E t, R^{2}=E t, 86 \%$

28n, $R^{1}=R^{2}=-\left(\mathrm{CH}_{2}\right)_{3}-, 72 \% \quad$ 28q, $R^{1}=\mathrm{Me}, \mathrm{R}^{2}=\mathrm{H}, 96 \%$

28o, $\mathrm{R}^{1}=\mathrm{Me}, \mathrm{R}^{2}=n-\mathrm{Pr}, 90 \% \quad$ 28r, $\mathrm{R}^{1}=\mathrm{Ph}, \mathrm{R}^{2}=i-\mathrm{Pr}, 35 \%$

Cyclizations involving cycled alkenes $\operatorname{PhCN}(0.075 \mathrm{M}), 100^{\circ} \mathrm{C}$

28m-r

28g, $R^{1}=P h, R^{2}=E t, 70 \%$ 28h, $R^{1}=4-\mathrm{BrC}_{6} \mathrm{H}_{4}, R^{2}=\mathrm{Et}, 64 \%$ 28i, $R^{1}=P h, R^{2}=H, 64 \%$

28s, $R^{1}=H, R^{2}=H, 95 \%$

28t, $R^{1}=H, R^{2}=$ Benzyl, $55 \%$

28u, $\mathrm{R}^{1}=\mathrm{Me}, \mathrm{R}^{2}=\mathrm{Me}, 75 \%$

Scheme 11 Copper(I) catalyzed Heck-like cyclizations of oxime esters towards 1-pyrrolines. 


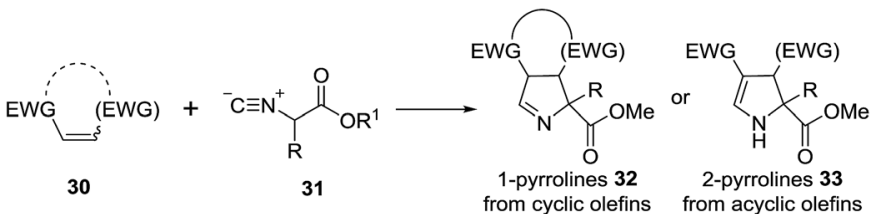

Scheme $12[3+2]$ Cycloaddition of isocyanoacetates with electrondeficient alkenes.

relies on an asymmetric Michael addition of ketiminoesters 26 to $\beta$-trifluoromethyl $\beta, \beta$-disubstituted enones 25 and subsequent hydrolytic cyclization. The optimized asymmetric conditions employed CuOAc as the catalyst and $(S, S)$ - ${ }^{\mathrm{i}} \mathrm{Pr}$-FOXAP as a chiral ligand in the presence of water (6 equiv.) to achieve this highly chemo-, diastereo-, and enantioselective reaction.

Bower et al. ${ }^{77}$ developed a copper(I)-catalyzed Heck-like cyclization of oxime esters as an effective alternative to Pdbased protocols (Scheme 11). One advantage of this methodology is that it works with less activated oxime esters such as pivaloyl oxime (28) as the starting material, instead of the more expensive $O$-pentafluorobenzoyl oximes that are required for the Pd protocol. The range of substrates which delivers 1-pyrrolines (29) includes pivaloyl oxime esters 28a-1 that possess pendant 1,2-disubstituted alkenes, the more heavily substituted 1,1-disubstituted alkenes $\mathbf{2 8 m}-\mathbf{r}$, and the cyclohexenes $\mathbf{2 8 s}-\mathbf{u}$. The proposed mechanism involves an intermediate that has iminyl radical character that triggers cyclization to form a C-N bond.

2.1.2. Synthesis of 1-pyrrolines by gold catalysis. A convergent preparation of pyrrolines consists of the formal [3+ 2] cycloaddition of isocyanoacetates $\mathbf{3 1}$ with electron-deficient alkenes 30 (Scheme 12). Base, or a metal complex accelerate significantly the reaction. ${ }^{78}$ With cyclic alkenes the cycloaddition gives the 1-pyrroline (32), whereas with acyclic alkenes the acyclic 2-pyrroline (33) is obtained.

In 2012, Carretero et al. reported ${ }^{79}$ a highly diastereoselective and enantioselective synthesis of 1-pyrrolines 36 by reaction of isocyanoacetates 34 with phenylmaleimide (35) using an $\mathrm{Au}(\mathrm{I})$ catalyst with a chiral DTBM-segphos ligand. The use of substituted isocyanoacetates led to 1-pyrrolines bearing a quaternary stereocenter at C-5 (Scheme 13). The reaction of $\alpha$ aryl-substituted and $\alpha$-alkyl-substituted isocyanoacetates gave

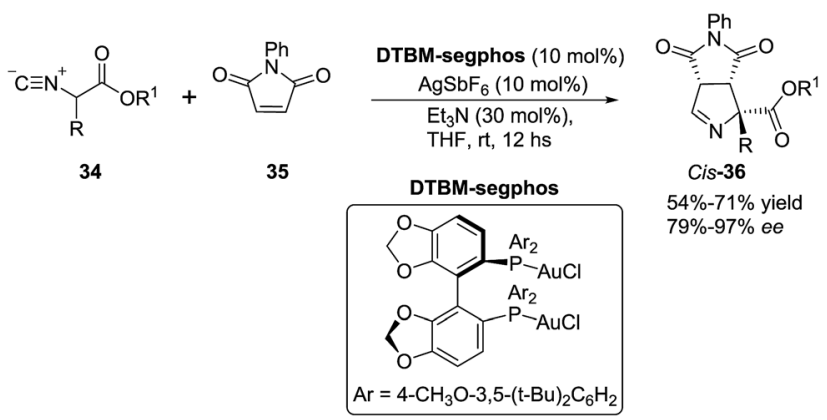

Scheme 13 Au-catalyzed asymmetric formal [3 + 2] cycloaddition of isocyanoacetates with maleimides.

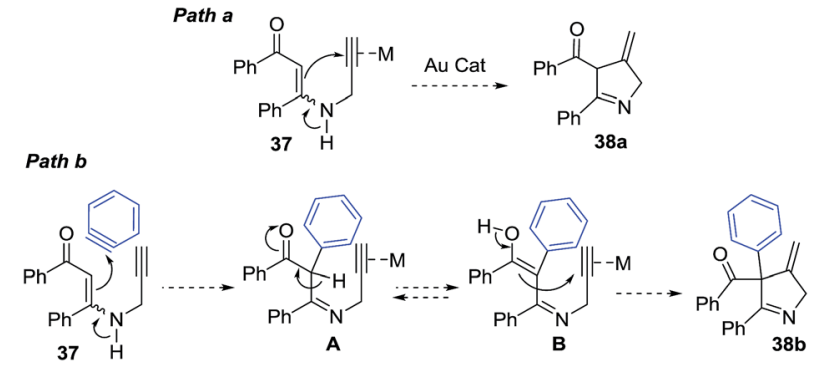

Scheme 14 Strategies for the conversion of $N$-propargylic $\beta$-enaminone 37 to 1 -pyrroline $38 \mathrm{~b}$ via gold catalysis.

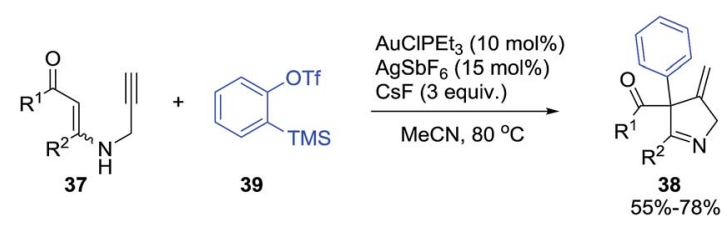

Scheme 15 Gold-catalyzed cyclization of $N$-propargylic $\beta$-enaminones 37 towards 1 -pyrrolines $38 \mathrm{~b}$ in presence of aryne precursor 39 .

the 1-pyrroline adduct as a single diastereomer and with high enantiocontrol. This methodology afforded 1-pyrrolines containing a quaternary stereocenter with complete cis diastereoselectivity (both carbonyl substituent oriented in the same direction of the pyrroline ring) and high enantioselectivity (up to $97 \%$ ee).

Karunakar et al. ${ }^{80}$ envisaged a route to 3-methylene-1pyrrolines from $N$-propargylic $\beta$-enaminones and arynes using gold catalysis. However, only recovered starting material was obtained from the attempted reaction of enaminone 37 and various gold catalysts (Scheme 14, path a). The failure of the reaction was attributed to the poor nucleophilicity of the enaminone or the weak electrophilic character of the propargylic functionality. Then, they had the innovative idea to use an external aryne to enable cyclization (path b). Using benzyne generated in situ and $\mathrm{AuCl}_{3} / \mathrm{AgSbF}_{6}$ as catalysts, they observed the formation of the 3-methylene-1-pyrroline $\mathbf{3 8 b}$ (Scheme 14). After a rigorous catalyst screening, they found that the combination of $\mathrm{AuCl} \cdot \mathrm{PEt}_{3}(10 \mathrm{~mol} \%)$ and $\mathrm{AgSbF}_{6}(15 \mathrm{~mol} \%)$ in presence of the aryne precursor 39 in $\mathrm{CH}_{3} \mathrm{CN}$ at $80^{\circ} \mathrm{C}$ gave the best yields (Scheme 15). Evaluation of the scope of the reaction using different $N$-propargylic $\beta$-enaminones demonstrated that enaminone with electron-donating groups increased the yield of the cyclisation products.

Our group $^{81}$ developed a versatile strategy to obtain a variety of disubstituted 1-pyrrolines $\mathbf{4 2}$ through a gold-catalyzed $\mathrm{N}$ -

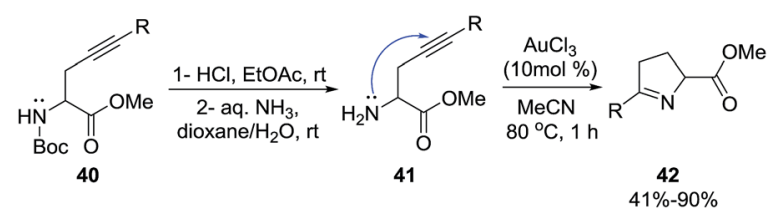

Scheme 16 Gold-catalyzed C-N functionalization of alkynyl amino acids derivatives. 

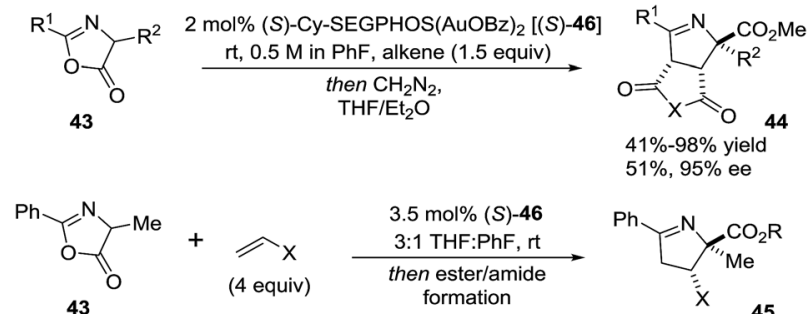
$41 \%-98 \%$ yield
$51 \%, 95 \%$ ee

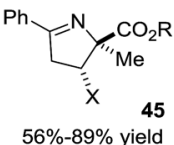

$76 \%, 99 \%$ yie
Scheme 17 Enantioselective gold(I)-catalyzed reactions of azlactones

cycloisomerization from alkyne-containing amino acids 40 (Scheme 16). Of the gold catalysts examined, the most effective for this $\mathrm{C}-\mathrm{N}$ functionalization was $\mathrm{AuCl}_{3}$.

Although this transformation can be performed with other transition metals and with Brønsted acids, $\mathrm{AuCl}_{3}$ is the superior catalyst as it provides a broader scope and better yields. This cycloisomerization of aryl-substituted alkynyl-containing amino acids proceeds exclusively via $\mathrm{Au}(\mathrm{III})$-catalyzed 5-endo-dig $\mathrm{N}$ cyclization. Aryl-substituted alkynyl amino acids bearing electron withdrawing groups provide excellent yields (typically 90\%) of the $\mathrm{C}-\mathrm{N}$ functionalized product whilst derivatives carrying electron-donating groups furnish lower yields (typically 55\%). Terminal alkynes fail to afford the pyrroline, they are unreactive under these conditions.

Toste $e t a .^{82}$ evaluated the gold-catalyzed reactions between azlactones $\mathbf{4 3}$ and electron-deficient alkenes (such as maleimide and maleic anhydrides, or monosubstituted alkenes) to give stereoselectively the products (44 and $\mathbf{4 5}$, respectively) of 1,3dipolar cycloaddition (Scheme 17). The use of $\mathrm{C}_{2}$-symmetric bis(phosphinegold(I) carboxylate) complexes (S-46) provided good to excellent diastereo- and enantioselectivity to afford 1-pyrrolines 44 and 45. The authors proposed a mechanism in which the gold complexes activate pro-nucleophiles to catalyze the cycloaddition, rather than the more typical mechanism of activation of the carbon-carbon $\pi$-bond toward nucleophilic addition.

Zhu et al. reported a copper-catalyzed domino cyclization/ trifluoromethylthiolation of monosubstituted or 1,1 disubstituted olefins $\mathbf{4 7}$ leading to $\mathrm{SCF}_{3}$-substituted 1-pyrrolines $\mathbf{4 8}$ in 60$90 \%$ yields (Scheme 18). ${ }^{83} \mathrm{AgSCF}_{3}$ is the reagent of choice as $\mathrm{SCF}_{3}$ source. Benzoyloxy group and $\mathrm{Cu}(\mathrm{OAc})_{2}$ proved to be the best oxime leaving group and catalyst for this transformation. This

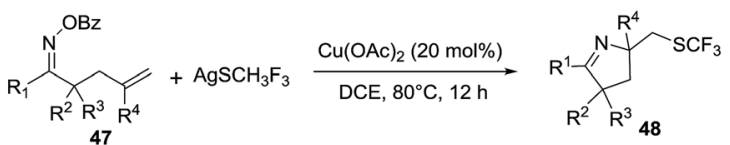

48a, $R^{1}=\mathrm{Ph} ; \mathrm{R}^{2}=\mathrm{H} ; \mathrm{R}^{3}=\mathrm{H} ; \mathrm{R}^{4}=\mathrm{H} ; 81 \% \quad 48 \mathrm{i}, \mathrm{R}^{1}=2$-naphthyl; $\mathrm{R}^{2}=\mathrm{H} ; \mathrm{R}^{3}=\mathrm{H} ; \mathrm{R}^{4}=\mathrm{H} ; 65 \%$ 48b, $R^{1}=4-M_{e} \mathrm{OC}_{6} \mathrm{H}_{4} ; \mathrm{R}^{2}=\mathrm{H} ; \mathrm{R}^{3}=\mathrm{H} ; \mathrm{R}^{4}=\mathrm{H} ; 75 \% 48 \mathrm{j}, \mathrm{R}^{1}=2$-thienyl; $\mathrm{R}^{2}=\mathrm{H} ; \mathrm{R}^{3}=\mathrm{H} ; \mathrm{R}^{4}=\mathrm{H} ; 60 \%$ 48c, $R^{1}=4-M_{e} \mathrm{C}_{6} \mathrm{H}_{4} ; \mathrm{R}^{2}=\mathrm{H} ; \mathrm{R}^{3}=\mathrm{H} ; \mathrm{R}^{4}=\mathrm{H} ; 71 \% \quad 48 \mathrm{k}, \mathrm{R}^{1}=\mathrm{Ph} ; \mathrm{R}^{2}=\mathrm{H} ; \mathrm{R}^{3}=\mathrm{H} ; \mathrm{R}^{4}=\mathrm{Me} ; 70 \%$ 48d, $R^{1}=4-\mathrm{ClC}_{6} \mathrm{H}_{4} ; \mathrm{R}^{2}=\mathrm{H} ; \mathrm{R}^{3}=\mathrm{H} ; \mathrm{R}^{4}=\mathrm{H} ; 70 \% \quad 48 \mathrm{I}, \mathrm{R}^{1}=\mathrm{Ph} ; \mathrm{R}^{2}=\mathrm{Me} ; \mathrm{R}^{3}=\mathrm{Me} ; \mathrm{R}^{4}=\mathrm{H} ; 73 \%$ 48e, $\mathrm{R}^{1}=3-\mathrm{MeOC}_{6} \mathrm{H}_{4} ; \mathrm{R}^{2}=\mathrm{H} ; \mathrm{R}^{3}=\mathrm{H} ; \mathrm{R}^{4}=\mathrm{H} ; 65 \% 48 \mathrm{~m}, \mathrm{R}^{1}=\mathrm{Ph} ; \mathrm{R}^{2} ; \mathrm{R}^{3}=$ cyclohexyl; $\mathrm{R}^{4}=\mathrm{H} ; 75 \%$ 48f, $R^{1}=3-\mathrm{ClC}_{6} \mathrm{H}_{4} ; \mathrm{R}^{2}=\mathrm{H} ; \mathrm{R}^{3}=\mathrm{H} ; \mathrm{R}^{4}=\mathrm{H} ; 89 \% . \quad 48 n, \mathrm{R}^{1}=\mathrm{Ph} ; \mathrm{R}^{2}=\mathrm{Me} ; \mathrm{R}^{3}=\mathrm{Me} ; \mathrm{R}^{4}=\mathrm{Me} ; 63 \%$ 48g, $R^{1}=2-\mathrm{ClC}_{6} \mathrm{H}_{4} ; \mathrm{R}^{2}=\mathrm{H} ; \mathrm{R}^{3}=\mathrm{H} ; \mathrm{R}^{4}=\mathrm{H} ; 74 \% \quad 480, \mathrm{R}^{1}=\mathrm{CH}_{2} \mathrm{CH}_{2} \mathrm{C}_{6} \mathrm{H}_{4} ; \mathrm{R}^{2}=\mathrm{H} ; \mathrm{R}^{3}=\mathrm{H} ; \mathrm{R}^{4}=\mathrm{H} ; 61 \%$ 48h, $R^{1}=3,4-M_{e O C} H_{4} ; R^{2}=H ; R^{3}=H ; R^{4}=H ; 67 \%$

Scheme 18 Copper-catalyzed synthesis of $\mathrm{SCF}_{3}$-containing 1-pyrrolines 48

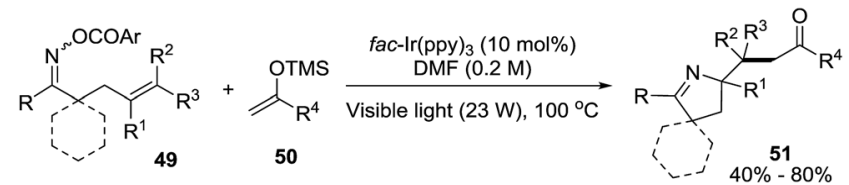

Scheme 19 Visible-light-promoted carboimination of unactivated alkenes towards 1-pyrrolines.

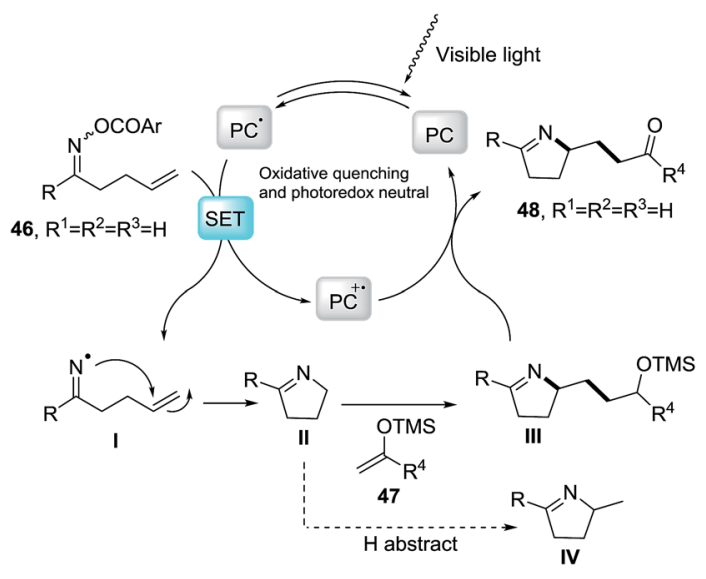

Scheme 20 Proposed reaction mechanism.

reaction involves the $\mathrm{N}-\mathrm{O}$ cleavage of benzoyl oximes and subsequent alkene difunctionalization and tolerates electron-donating and electron-withdrawing groups on the aryl-substituted $O$-acyl oximes $\left(\mathrm{R}^{1}=\mathrm{Ar}, \mathbf{4 8} \mathbf{-}-\mathbf{h}\right)$ as well as alkyl-substituted $O$-acyl oxime. $\mathrm{R}^{1}=$ naphthyl and thienyl delivered the $\mathrm{SCF}_{3}$-containing 1-pyrrolines in good yields. The reaction is also compatible with alkyl moieties as the substituents $\mathrm{R}^{2}$ and $\mathrm{R}^{3}$.

2.1.3. Synthesis of 1-pyrrolines by iridium catalysis. Loh et al. ${ }^{84}$ developed a strategy based on the iminyl-radical formation for the creation of the $\mathrm{C}-\mathrm{N}$ bond of the pyrroline (Scheme 19). The iminyl-radical is generated from the $O$-acyl oxime derivatives 49 in presence of a photocatalyst such as fac-[Ir$\left.(\mathrm{ppy})_{3}\right]$ and visible light. The cascade reaction takes place through an intramolecular 5-exo cyclization followed by intermolecular carbon radical trapping with silyl enol ether derivatives 50 (Scheme 20). The procedure allows easy access to densely functionalized pyrroline derivatives $\mathbf{5 1}$. The use of silyl enol ethers as coupling partners regenerate the photocatalyst without necessity of an external reductant, while introducing the synthetically useful ketone functionalities. The procedure shows tolerance to a broad range of functionalities and enables diverse substitution patterns with respect to both the starting oxime derivative and the silyl enol ether. Different densely functionalized pyrrolines are obtained in moderate to good yields (typically $40-80 \%$ ).

Then, the same group reported a modification of previous protocol adding $N, N^{\prime}$-dimethylpropylene urea (DMPU) as solvent, reductant and $\mathrm{H}$ donor. ${ }^{85}$ As result, a visible-light-promoted hydroimination of unactivated alkenes $\mathbf{5 2}$ catalyzed by iridium towards the synthesis of 1-pyrrolines $\mathbf{5 3}$ was accomplished (Scheme 21). As in the previous report, the procedure shows 


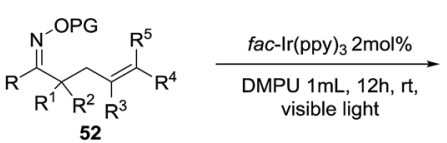

53a, $\mathrm{R}=\mathrm{Ph} ; \mathrm{R}^{1}=\mathrm{H} ; \mathrm{R}^{2}=\mathrm{H} ; \mathrm{R}^{3}=\mathrm{H} ; \mathrm{R}^{4}=\mathrm{H} ; \mathrm{R}^{5}=\mathrm{H} ; 72 \%$ 53b, R=4-MeC ${ }_{6} \mathrm{H}_{4} ; \mathrm{R}^{1}=\mathrm{H} ; \mathrm{R}^{2}=\mathrm{H} ; \mathrm{R}^{3}=\mathrm{H} ; \mathrm{R}^{4}=\mathrm{H} ; \mathrm{R}^{5}=\mathrm{H} ; 77 \%$ 53c, $\mathrm{R}=2-\mathrm{MeC}_{6} \mathrm{H}_{4} ; \mathrm{R}^{1}=\mathrm{H} ; \mathrm{R}^{2}=\mathrm{H} ; \mathrm{R}^{3}=\mathrm{H} ; \mathrm{R}^{4}=\mathrm{H} ; \mathrm{R}^{5}=\mathrm{H} ; 64 \%$ 53d, R=4-MeOC $6 \mathrm{H}_{4} ; \mathrm{R}^{1}=\mathrm{H} ; \mathrm{R}^{2}=\mathrm{H} ; \mathrm{R}^{3}=\mathrm{H} ; \mathrm{R}^{4}=\mathrm{H} ; \mathrm{R}^{5}=\mathrm{H} ; 90 \%$ 53e, $\mathrm{R}=4-\mathrm{FC}_{6} \mathrm{H}_{4} ; \mathrm{R}^{1}=\mathrm{H} ; \mathrm{R}^{2}=\mathrm{H} ; \mathrm{R}^{3}=\mathrm{H} ; \mathrm{R}^{4}=\mathrm{H} ; \mathrm{R}^{5}=\mathrm{H} ; 77 \%$ 53f, $\mathrm{R}=3-\mathrm{FC}_{6} \mathrm{H}_{4} ; \mathrm{R}^{1}=\mathrm{H} ; \mathrm{R}^{2}=\mathrm{H} ; \mathrm{R}^{3}=\mathrm{H} ; \mathrm{R}^{4}=\mathrm{H} ; \mathrm{R}^{5}=\mathrm{H} ; 89 \%$ 53g, R=4-ClC ${ }_{6} \mathrm{H}_{4} ; \mathrm{R}^{1}=\mathrm{H} ; \mathrm{R}^{2}=\mathrm{H} ; \mathrm{R}^{3}=\mathrm{H} ; \mathrm{R}^{4}=\mathrm{H} ; \mathrm{R}^{5}=\mathrm{H} ; 96 \%$ 53h, R=3-ClC $\mathrm{H}_{4} ; \mathrm{R}^{1}=\mathrm{H} ; \mathrm{R}^{2}=\mathrm{H} ; \mathrm{R}^{3}=\mathrm{H} ; \mathrm{R}^{4}=\mathrm{H} ; \mathrm{R}^{5}=\mathrm{H} ; 87 \%$ $53 i, R=2-\mathrm{ClC}_{6} \mathrm{H}_{4} ; \mathrm{R}^{1}=\mathrm{H} ; \mathrm{R}^{2}=\mathrm{H} ; \mathrm{R}^{3}=\mathrm{H} ; \mathrm{R}^{4}=\mathrm{H} ; \mathrm{R}^{5}=\mathrm{H} ; 96 \%$ 53j,R=4-BrC ${ }_{6} \mathrm{H}_{4} ; \mathrm{R}^{1}=\mathrm{H} ; \mathrm{R}^{2}=\mathrm{H} ; \mathrm{R}^{3}=\mathrm{H} ; \mathrm{R}^{4}=\mathrm{H} ; \mathrm{R}^{5}=\mathrm{H} ; 81 \%$ $53 \mathrm{k}, \mathrm{R}=4-\mathrm{CF}_{3} \mathrm{C}_{6} \mathrm{H}_{4} ; \mathrm{R}^{1}=\mathrm{H} ; \mathrm{R}^{2}=\mathrm{H} ; \mathrm{R}^{3}=\mathrm{H} ; \mathrm{R}^{4}=\mathrm{H} ; \mathrm{R}^{5}=\mathrm{H} ; 66 \%$ 53I, R=3,5-diCF ${ }_{3} \mathrm{C}_{6} \mathrm{H}_{4} ; \mathrm{R}^{1}=\mathrm{H} ; \mathrm{R}^{2}=\mathrm{H} ; \mathrm{R}^{3}=\mathrm{H} ; \mathrm{R}^{4}=\mathrm{H} ; \mathrm{R}^{5}=\mathrm{H} ; 69 \%$ 53m, R=4-BocNHC $\mathrm{H}_{4} ; \mathrm{R}^{1}=\mathrm{H} ; \mathrm{R}^{2}=\mathrm{H} ; \mathrm{R}^{3}=\mathrm{H} ; \mathrm{R}^{4}=\mathrm{H} ; \mathrm{R}^{5}=\mathrm{H} ; 86 \%$ 53n, R=4-MeCO $\mathrm{C}_{6} \mathrm{H}_{4} ; \mathrm{R}^{1}=\mathrm{H} ; \mathrm{R}^{2}=\mathrm{H} ; \mathrm{R}^{3}=\mathrm{H} ; \mathrm{R}^{4}=\mathrm{H} ; \mathrm{R}^{5}=\mathrm{H} ; 98 \%$ 53o, $\mathrm{R}=4-\mathrm{PhNHCOC}_{6} \mathrm{H}_{4} ; \mathrm{R}^{1}=\mathrm{H} ; \mathrm{R}^{2}=\mathrm{H} ; \mathrm{R}^{3}=\mathrm{H} ; \mathrm{R}^{4}=\mathrm{H} ; \mathrm{R}^{5}=\mathrm{H} ; 90 \%$ 53p, R=4-CNC ${ }_{6} \mathrm{H}_{4} ; \mathrm{R}^{1}=\mathrm{H} ; \mathrm{R}^{2}=\mathrm{H} ; \mathrm{R}^{3}=\mathrm{H} ; \mathrm{R}^{4}=\mathrm{H} ; \mathrm{R}^{5}=\mathrm{H} ; 86 \%$ 53q, R=2-Thienyl; $R^{1}=H ; R^{2}=H ; R^{3}=H ; R^{4}=H ; R^{5}=H ; 56 \%{ }^{[a]}, 59 \%{ }^{[b]}$ 53r, R=2-naphthyl; $\mathrm{R}^{1}=\mathrm{H} ; \mathrm{R}^{2}=\mathrm{H} ; \mathrm{R}^{3}=\mathrm{H} ; \mathrm{R}^{4}=\mathrm{H} ; \mathrm{R}^{5}=\mathrm{H} ; 48 \%$ 53s, $R=P h ; R^{1}=M e ; R^{2}=M e ; R^{3}=H ; R^{4}=H ; R^{5}=H ; 80 \%$ 53t, $R=P h ; R^{1}=M e ; R^{2}=M e ; R^{3}=M e ; R^{4}=H ; R^{5}=H ; 90 \%$ 53u, $\mathrm{R}=\mathrm{Ph} ; \mathrm{R}^{1}=\mathrm{Me} ; \mathrm{R}^{2}=\mathrm{Me} ; \mathrm{R}^{3}=\mathrm{H} ; \mathrm{R}^{4}=\mathrm{Me} ; \mathrm{R}^{5}=\mathrm{Me} ; 78 \%$ 53v, $\mathrm{R}=\mathrm{C}_{6} \mathrm{H}_{4} \mathrm{OCH}_{2}-\mathrm{R}^{1}=\mathrm{H} ; \mathrm{R}^{2}=\mathrm{H} ; \mathrm{R}^{3}=\mathrm{H} ; \mathrm{R}^{4}=\mathrm{H} ; \mathrm{R}^{5}=\mathrm{H} ; 40 \%$

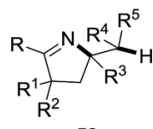

53
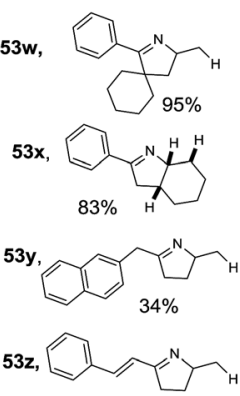
$14 \%$

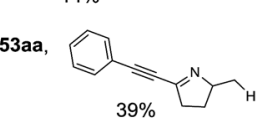

[a]:Z-configuration substrate. [b]:E-configuration substrate.
Scheme 21 Intramolecular cycloaddition of 52 by iridium catalyst.

a broad scope of functionalities and tolerates diverse substitution patterns of the aryl $O$-acyl oximes to afford 1-pyrrolines $\mathrm{XX}$ in good to excellent yields (64-98\%). However, a diminished yield was observed when alkyl, naphthyl, alkenyl and alkynyl derived $O$-acyl oximes (R) were employed as substrates (14-48\%).

Studer $e t$ al. applied visible light to promote the generation of iminyl-radicals by a photoredox decarboxylation of $\alpha$-iminooxy propionic acids $\mathbf{5 4}$ towards the synthesis of 1-pyrrolines 56 (Scheme 22). ${ }^{86}$ The reaction mixture between $\alpha$-imino-oxy propionic acids 54 and olefins with electron withdrawing groups 55 in the presence of $1 \mathrm{~mol} \%$ of $\operatorname{Ir}\left(\mathrm{dFCF}_{3} \text { ppy }\right)_{2}$-(dtbbpy) $\mathrm{PF}_{6}$ - a photoredox catalyst - and $\mathrm{K}_{3} \mathrm{PO}_{4}$ in 1,2-dichloroethane is irradiated with blue LED light to produce 1-pyrrolines 56 in good to excellent yields (typically $42-91 \%$ ). The reaction takes place without any control of the diasteroselectivity. Different $\alpha, \beta$-unsaturated esters with substituents at the $\alpha$ and $\beta$ position, $\alpha, \beta$-unsaturated amides, phosphonate and ketones were efficient Michael acceptors (55). Regarding the iminyl radical precursor 54, phenyl groups with electron-rich or electron-poor substituents, 2-thienyl, 2-naphthyl and alkyl groups at $\mathrm{R}^{1}$ position were well tolerated. $\alpha$-Iminyl-oxy acids with substituents at $\mathrm{R}^{2}, \mathrm{R}^{3}, \mathrm{R}^{5}$ and $\mathrm{R}^{6}$ positions were good substrates.

Messerle et al. ${ }^{87}$ reported the synthesis of 1-pyrrolines via an intramolecular hydroamination of a series of alkynamines catalyzed by both iridium and rhodium complexes with bidentate $\mathrm{N}-$ $\mathrm{N}^{\prime}$ donor ligands (see Fig. 3, Schemes 44 and 45 in Subsection 2.1.7.: Synthesis of 1-pyrrolines by rhodium catalysis).

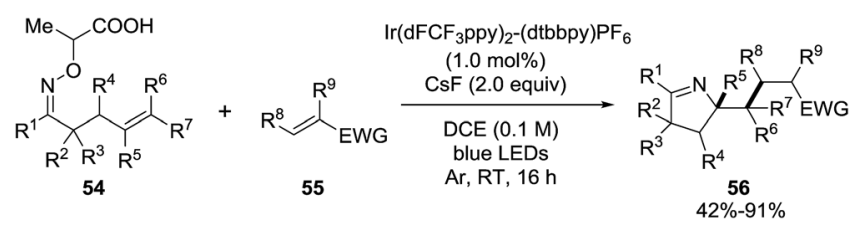

Scheme 22 Synthesis of 1-pyrrolines 56 using blue LEDs.

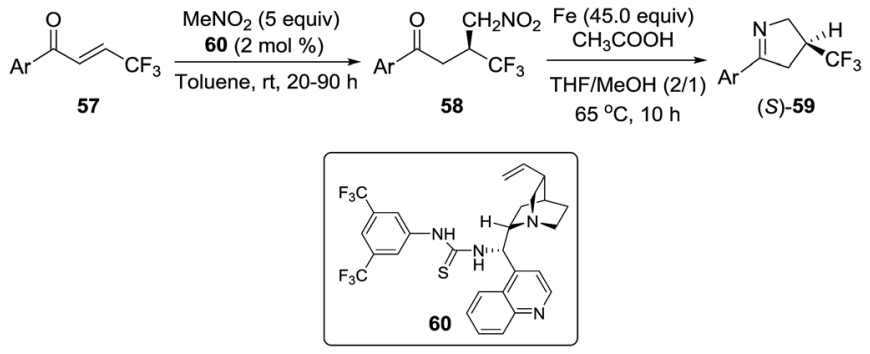

Scheme 23 One-pot enantioselective synthesis of 3-trifluoromethylated 1-pyrrolines (S)-59.

2.1.4. Synthesis of 1-pyrrolines by iron catalysis. In 2012, Shibata et $a l .{ }^{88}$ described the one-pot enantioselective synthesis of $\beta$-trifluoromethyl-substituted pyrrolines $\mathbf{5 9}$ by an asymmetric conjugate addition of nitromethane to $\beta$-trifluoromethylenones 57 catalyzed by a cinchona alkaloid-derived/thiourea (60), followed by an iron-mediated reduction/cyclization/dehydration sequence of the intermediate 58. Excellent yields and high enantioselectivities (up to $98 \%$ ee) were obtained (Scheme 23 ).

Trifluoromethylated 3,5-diaryl-pyrrolines (63) have attracted much attention because of their promising agrochemical activity as antiparasitics. Since this finding in 2005 , more than 7000 variants have been described across numerous patents. In related work Shibata et al. ${ }^{89}$ disclosed the asymmetric synthesis of 3-trifluoromethyl 3,5-diaryl-pyrrolines 63 using excess Fe(0) for the cyclization step (Scheme 24). The chiral starting materials for this reaction were made by the enantioselective conjugate addition (using a phase-transfer organocatalyst 64) of nitromethane with a variety of $\beta, \beta$-disubstituted enones (61). A family of eighteen 1,4 adducts 62 were obtained in excellent yields and enantioselectivities. Conversion of $\mathbf{6 2}$ into the trifluoromethylated 3,5-diaryl-pyrrolines $\mathbf{6 3}$ was carried out using excess iron in the presence of acetic acid for the nitro reduction/ cyclization/dehydration sequence, and without any loss of the enantiopurity. Transformation to the biologically important trifluoromethylated arylpyrrolines $\mathbf{6 3}$ were achieved from the nitromethane adduct $\mathbf{6 2}$ with high to excellent yields (typically $90 \%$ ) in a single step.

Yang et al..$^{90}$ envisaged oxime esters as electrophilic partners for iron catalysis (Scheme 25). Reductive cleavage of the oxime $\mathrm{N}-\mathrm{O}$ bond by iron generates useful iminyl radicals. ${ }^{91}$ Coupling

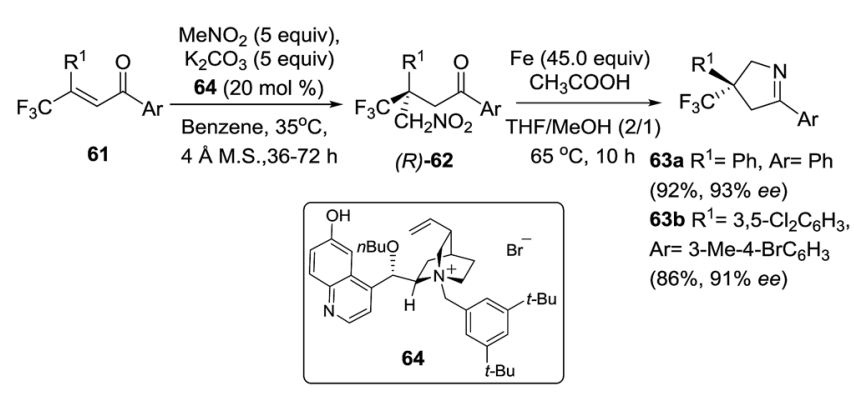

Scheme 24 Enantioselective conjugate addition of nitromethane to $\beta, \beta$-disubstituted enones 61 , followed by an iron-mediated reduction/ cyclization/dehydration sequence to 1-pyrrolines 63 . 
<smiles>C=C(C)CCC(=NOC(C)=O)c1ccccc1</smiles>

65

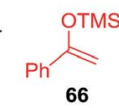

66
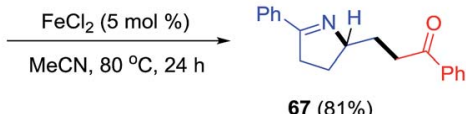

Scheme 25 Iron-catalyzed coupling of $O$-acyloximes with silyl enol ethers.

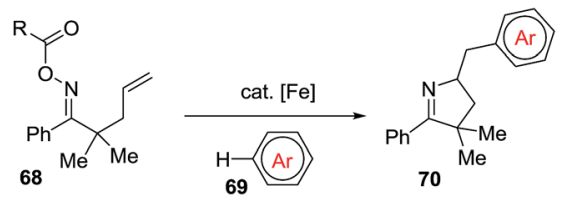

Scheme 26 Synthesis of 1-pyrrolines 70.

of iminyl radical derivatives of $\gamma, \delta$-unsaturated oxime (65) with silyl enol ether (66) lead to pyrroline 67 through an intramolecular $\mathrm{C}-\mathrm{N}$ bond formation.

The Oche and the Okamoto group disclosed an ironcatalyzed methodology to achieve 1-pyrrolines $\mathbf{7 0}$ that involved the formation of iminyl radicals from alkene-tethered oxime esters 68 and subsequent aminative cyclization and intermolecular homolytic aromatic substitution (Scheme 26). ${ }^{\mathbf{9 2}}$

The optimized conditions use $10 \mathrm{~mol} \%$ of $\mathrm{Fe}(\mathrm{OTf})_{2}$ and ligand $\mathbf{L} 1$ in presence of a great excess of arenes (150 equiv.). The mixture is heated a $120{ }^{\circ} \mathrm{C}$ for $12 \mathrm{~h}$.

The reaction tolerates different arenes 69 with electron-rich and electron-poor substituent, polycyclic aromatic compounds as well as nitrogen- or sulfur-containing heteroarenes. Regarding the scope of alkene-tethered oxime ester $68, \mathrm{R}=$ picolinoyl ester (68a-2-Py) and $\mathrm{R}=$ pivaloyl ester $(\mathbf{6 8 a}-t \mathrm{Bu})$ can be used with the picolinoyl ester giving better yields with substituted arenes. Oxime esters with substituted alkenes $\left(\mathrm{R}^{1} \neq\right.$ $\mathrm{H}$ and/or $\mathrm{R}^{2} \neq \mathrm{H}$ ) provided 1-pyrrolines in good yields. The reaction is applicable to oxime esters 68 with $R^{3}$ and $R^{4}=H$ or alkyl groups and $\mathrm{R}^{5}=$ heteroaryl, naphthyl or alkyl groups (Scheme 27). This transformation involving radical intermediates affords 1-pyrrolines 70 with moderate to good yields (36$71 \%)$.

2.1.5. Synthesis of 1-pyrrolines by nickel catalysis. Zard et $a{ }^{93}$ conceived of a method for the generation of iminyl radicals based on the reduction of oxime esters (71) with a combination of nickel powder and acetic acid (Scheme 28). The radical center on the nitrogen is intercepted intramolecularly by the well-positioned terminal alkene. Subsequent quenching by isopropanol gives pyrroline 72 . The authors proved this radical mechanism with addition of diphenyldiselenide (PhSeSePh), which led to selenide 73. On the other hand, when they replaced the unsubstituted terminal alkene of 71a/ b by a trisubstituted alkene of $\mathbf{7 4 a} / \mathbf{b}$, they obtained the isopropenyl alkene $76 \mathbf{a}$ and the tertiary acetate $75 \mathbf{a}$ in the case of 74a. When starting from $\mathbf{7 4 b}$, they observed only formation of 75b (Scheme 29). The authors determined that this mechanism is ionic, and not radical, with the following experiment. Reaction of the oxime acetates $\mathbf{7 4 a}$ and $\mathbf{7 4 b}$ containing an isopropylidene group with $\mathrm{Cu}(\mathrm{OAc})_{2} / \mathrm{AcOH}$ in refluxing $t$-butanol
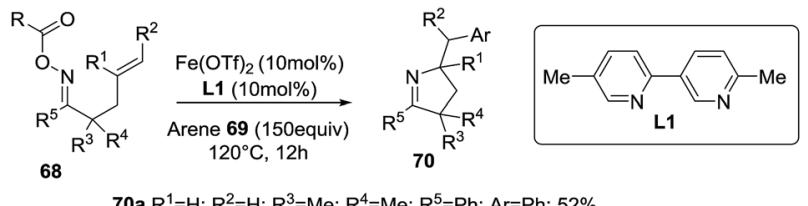

$70 a, R^{1}=H ; R^{2}=H ; R^{3}=M e ; R^{4}=M e ; R^{5}=P h ; A r=P h ; 52 \%$

$70 b, R^{1}=H ; R^{2}=H ; R^{3}=M e ; R^{4}=M e ; R^{5}=\mathrm{Ph} ; \mathrm{Ar}=\mathrm{ClC}_{6} \mathrm{H}_{4} ; 62 \%$ (o:m:p=68:24:8)

$70 c, R^{1}=H ; R^{2}=H ; R^{3}=M e ; R^{4}=M e ; R^{5}=P h ; A r=2,5-d i C l C_{6} H_{3} ; 70 \%$

70d, $R^{1}=\mathrm{H} ; \mathrm{R}^{2}=\mathrm{H} ; \mathrm{R}^{3}=\mathrm{Me} ; \mathrm{R}^{4}=\mathrm{Me} ; \mathrm{R}^{5}=\mathrm{Ph} ; \mathrm{Ar}=2,3-\mathrm{diClC}_{6} \mathrm{H}_{3} ; 43 \%$

$70 \mathrm{e}, \mathrm{R}^{1}=\mathrm{H} ; \mathrm{R}^{2}=\mathrm{H} ; \mathrm{R}^{3}=\mathrm{Me} ; \mathrm{R}^{4}=\mathrm{Me} ; \mathrm{R}^{5}=\mathrm{Ph} ; \mathrm{Ar}=3,4-\mathrm{diClC}_{6} \mathrm{H}_{3} ; 25 \%$

$70 f, R^{1}=H ; R^{2}=H ; R^{3}=M e ; R^{4}=M e ; R^{5}=P h ; A r=2,4,6-$ triClC $_{6} \mathrm{H}_{2} ; 36 \%$

$70 \mathrm{~g}, \mathrm{R}^{1}=\mathrm{H} ; \mathrm{R}^{2}=\mathrm{H} ; \mathrm{R}^{3}=\mathrm{Me} ; \mathrm{R}^{4}=\mathrm{Me} ; \mathrm{R}^{5}=\mathrm{Ph} ; \mathrm{Ar}=2,5-\mathrm{diBrC}_{6} \mathrm{H}_{3} ; 60 \%$

$70 h, R^{1}=H ; R^{2}=H ; R^{3}=M e ; R^{4}=M e ; R^{5}=P h ; A r=2,5-d i F C_{6} H_{3} ; 46 \%$

$70 \mathrm{i}, \mathrm{R}^{1}=\mathrm{H} ; \mathrm{R}^{2}=\mathrm{H} ; \mathrm{R}^{3}=\mathrm{Me} ; \mathrm{R}^{4}=\mathrm{Me} ; \mathrm{R}^{5}=\mathrm{Ph} ; \mathrm{Ar}=2-\mathrm{CN}-5-\mathrm{CO}_{2} \mathrm{MeC}_{6} \mathrm{H}_{3} ; 58 \%$

$70 \mathrm{j}, \mathrm{R}^{1}=\mathrm{H} ; \mathrm{R}^{2}=\mathrm{H} ; \mathrm{R}^{3}=\mathrm{Me} ; \mathrm{R}^{4}=\mathrm{Me} ; \mathrm{R}^{5}=\mathrm{Ph} ; 2-\mathrm{CN}-5-\mathrm{ClC}_{6} \mathrm{H}_{3} ; 49 \%$

$70 k, R^{1}=H ; R^{2}=H ; R^{3}=M e ; R^{4}=M e ; R^{5}=\mathrm{Ph} ; A r=P \mathrm{PhC}_{6} \mathrm{H}_{4} ; 70 \%$ (o:m:p=51:39:10) $70 \mathrm{I}, \mathrm{R}^{1}=\mathrm{H} ; \mathrm{R}^{2}=\mathrm{H} ; \mathrm{R}^{3}=\mathrm{Me} ; \mathrm{R}^{4}=\mathrm{Me} ; \mathrm{R}^{5}=\mathrm{Ph} ; \mathrm{Ar}=2-\mathrm{Cl}-5-\left(p-\mathrm{ClC}_{6} \mathrm{H}_{4} \mathrm{CO}-\mathrm{C}_{6} \mathrm{H}_{3} ; 67 \%\right.$

$70 \mathrm{~m}, \mathrm{R}^{1}=\mathrm{H} ; \mathrm{R}^{2}=\mathrm{H} ; \mathrm{R}^{3}=\mathrm{Me} ; \mathrm{R}^{4}=\mathrm{Me} ; \mathrm{R}^{5}=\mathrm{Ph} ; \mathrm{Ar}=1$-naphthyl; $61 \% 56 \%$

$70 \mathrm{n}, \mathrm{R}^{1}=\mathrm{H} ; \mathrm{R}^{2}=\mathrm{H} ; \mathrm{R}^{3}=\mathrm{Me} ; \mathrm{R}^{4}=\mathrm{Me} ; \mathrm{R}^{5}=\mathrm{Ph} ; \mathrm{Ar}=2$-naphthyl; $11 \% 13 \%$
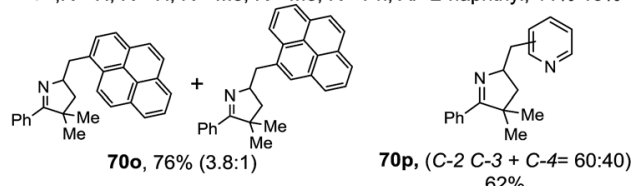

$62 \%$ $(C-2$ C-3 + C-4 $=88: 12)$

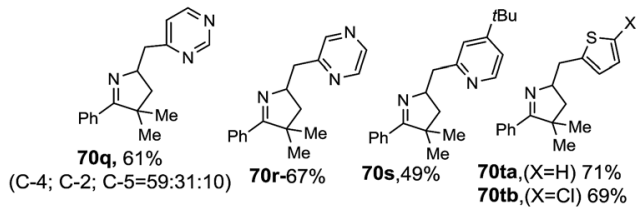

$70 \mathrm{u}, \mathrm{R}^{1}=\mathrm{Me} ; \mathrm{R}^{2}=\mathrm{H} ; \mathrm{R}^{3}=\mathrm{Me} ; \mathrm{R}^{4}=\mathrm{Me} ; \mathrm{R}^{5}=\mathrm{Ph} ; \mathrm{Ar}=2$-thienyl; $48 \%$

$70 \mathrm{v}, \mathrm{R}^{1}=\mathrm{Me} ; \mathrm{R}^{2}=\mathrm{Me} ; \mathrm{R}^{3}=\mathrm{Me} ; \mathrm{R}^{4}=\mathrm{Me} ; \mathrm{R}^{5}=\mathrm{Ph} ; \mathrm{Ar}=2$-thienyl; $59 \%$ (d. $\mathrm{r}=1.2: 1.0$ )

70x, $\mathrm{R}^{1}=\mathrm{H} ; \mathrm{R}^{2}=\mathrm{H} ; \mathrm{R}^{3}=\mathrm{H} ; \mathrm{R}^{4}=\mathrm{H} ; \mathrm{R}^{5}=\mathrm{Ph} ; \mathrm{Ar}=2$-thienyl; $64 \%$

$70 y, R^{1}=H ; R^{2}=H ; R^{3,4}=$ cyclohexyl; $R^{5}=P h ; A r=2$-thienyl; $64 \%$

$70 z, R^{1}=H ; R^{2}=H ; R^{3}=M e ; R^{4}=M e ; R^{5}=2$-pyridyl; Ar=2-thienyl; $57 \%$

70ab, $\mathrm{R}^{1}=\mathrm{H} ; \mathrm{R}^{2}=\mathrm{H} ; \mathrm{R}^{3}=\mathrm{Me} ; \mathrm{R}^{4}=\mathrm{Me} ; \mathrm{R}^{5}=2$-naphthyl; $\mathrm{Ar}=2$-thienyl; $59 \%$

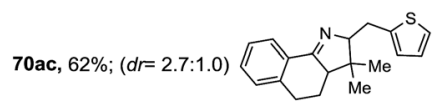

70ad, $\mathrm{R}^{1}=\mathrm{H} ; \mathrm{R}^{2}=\mathrm{H} ; \mathrm{R}^{3}=\mathrm{H} ; \mathrm{R}^{4}=\mathrm{H} ; \mathrm{R}^{5}=\mathrm{CH}_{2} \mathrm{CH}_{2} \mathrm{Ph} ; \mathrm{Ar}=2$-thienyl; $48 \%$

Scheme 27 Aminative cyclization/intermolecular homolytic aromatic substitution involving iminyl radicals towards 1-pyrrolines 70 .

gave the 1-pyrrolines $77 \mathbf{a}$ and $7 \mathbf{7 b}$ with an isopropenyl sidechain with very good yields (Scheme 30). Evaluation of other metallic salts for this reaction showed that from $\mathbf{7 4 a} \mathrm{FeCl}_{3}$ / $\mathrm{AcOH}$ in $t$-butanol at room temperature gave the acetate 75a and the alcohol 78. When they treated oxime 71a with $\mathrm{FeCl}_{3}$ in $t$ butanol in the absence of acetic acid (also at room temperature) they obtained the chloride 79 (64\% yield) and a small amount of the alcohol 80 ( $8 \%$ yield) as the products.

The scope of this $\mathrm{FeCl}_{3}$-promoted ionic transformation in $t$ butanol was examined with and without the presence of acetic

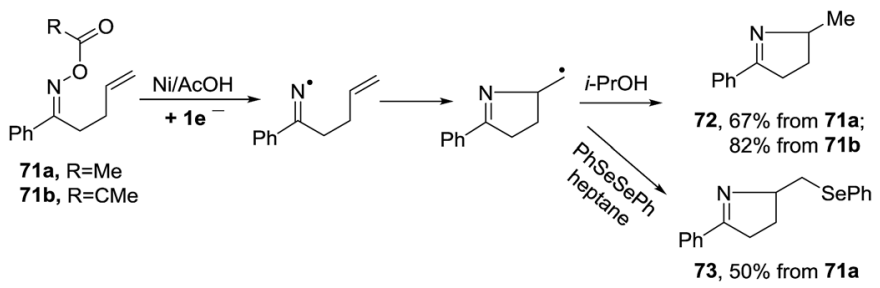

Scheme 28 Dissolving nickel metal mediated generation of iminyls from oxime esters. 


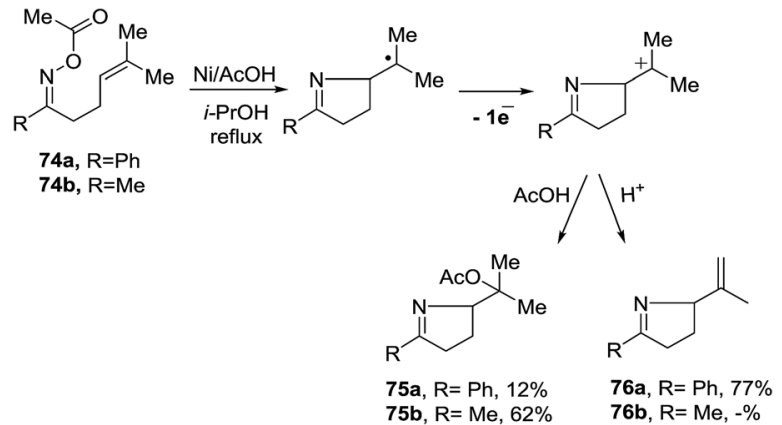

Scheme 29 Unexpected transformations of an unsaturated oxime acetate.
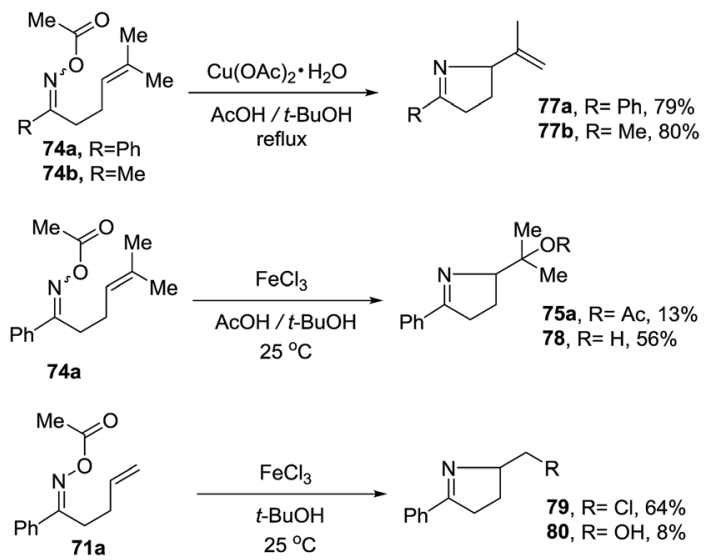

Scheme 30 Cupric and ferric ion-mediated cyclizations of unsaturated oxime acetates.

acid (Table 1). While several substitution patterns on the alkene were well tolerated, only the oxime acetate derived from aromatic ketones gave the 1-pyrrolines. The case of oxime acetate $\mathbf{9 0}$ derived from a methyl ketone is an exception since other oxime acetate derived from aliphatic ketones produced complex mixtures.

A possible mechanism is given in Scheme 31. The nitrogen from the oxime and the oxygen of ester form an intermediate complex with the metal ion, probably in equilibrium with the enamine that reacts via transition state $\mathbf{1 0 1}$ wherein acetate leaves as the weak $\mathrm{N}-\mathrm{O}$ bond is broken by nucleophilic attack of the alkene. Electron-rich alkenes are therefore better nucleophiles and improve the reaction. With $\mathrm{Cu}(\mathrm{II})$, an alkene is formed by abstraction of a proton (path a). With the $\mathrm{Fe}(\mathrm{III})$ of $\mathrm{FeCl}_{3}$, chloride anion competes as a nucleophile to give the chloride as a final product (path b).

Unlike palladium catalyzed cross-coupling reactions that suffer from $\beta$-H-elimination, cross-coupling reactions catalyzed with $\mathrm{Ni}$ are not susceptible to $\beta$-H-elimination. This feature was exploited by Selander $e t$ al. to developed a Nickel-catalyzed 1,2aminoarylation of oxime ester-tethered alkenes with boronic acids (Scheme 32). ${ }^{94}$ By reacting $\gamma, \delta$-unsaturated oxime esters 102 with boronic acids 103 in presence of $\mathrm{NiBr}_{2}(20 \mathrm{~mol} \%), \mathrm{Et}_{3} \mathrm{~N}$ (10 equiv.) and ligands $\mathbf{L} 2$ or $\mathbf{L} 3(20 \mathrm{~mol} \%)$ in dioxane at $90{ }^{\circ} \mathrm{C}, 1-$ pyrrolines 104 were obtained with moderate to good yields (31-
Table 1 Formation of chloroalkyl-1-pyrrolines 90-100 from unsaturated oxime acetates $81-90$ mediated by $\mathrm{FeCl}_{3}$

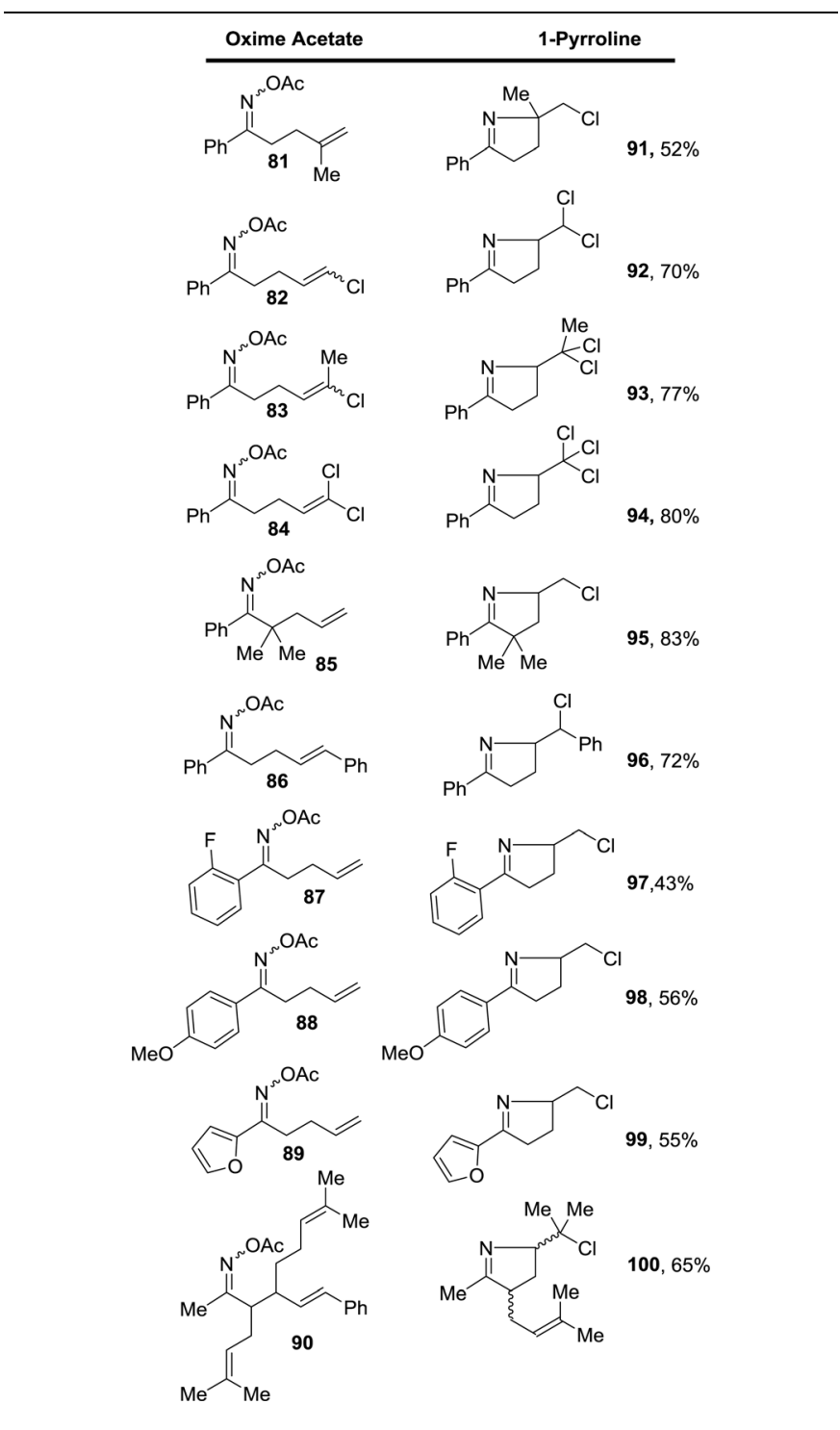

$82 \%)$. The reaction tolerates well several substituted arylboronic acids with electron withdrawing groups or electron donor groups and fused aromatic, heteroaromatic and vinylic boronic acids $\left(\mathrm{R}^{4}=\mathrm{Ar}\right.$, hetAr, vinyl). Aryl and heteroaryl oxime esters proceed well to 1-pyrrolines $\left(\mathrm{R}^{1}=\mathrm{Ar}\right.$, HetAr). The reaction is also suitable for non-terminal and cyclic alkenes. This nickelcatalyzed protocol is an excellent alternative to palladium catalyzed cross-coupling reactions (see Subsection 2.1.6.: Synthesis of 1-pyrrolines by palladium catalysis).

Recently, Wang et al. disclosure a Ni-biquinoline-catalyzed synthesis of multisubstituted 1-pyrrolines 107 through a reductive 1,2-iminoacylation of alkenes (Scheme 33). ${ }^{95}$ Oxime esters incorporating a pendant terminal olefinic unit 105 were reacted with electrophilic acylating reagents like acid chlorides (106b) or anhydrides (106a) and zinc as a reductive agent to generate 1pyrrolines 107 in yields ranging from 46 to $98 \%$. Aromatic and heteroaromatic groups in position $\mathrm{R}^{1}$ are well tolerated. Also, 

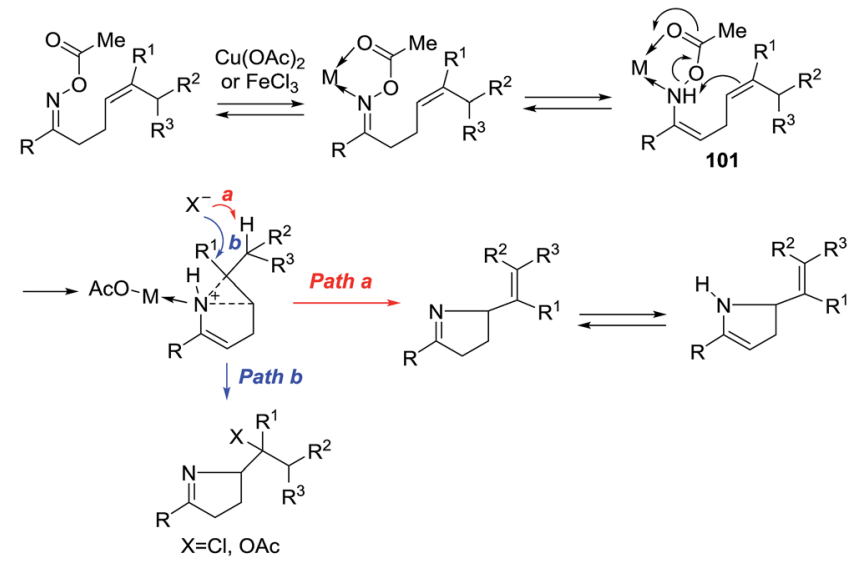

Scheme 31 Possible mechanism.

oxime esters with $R^{2}=H$ and monosubstituted alkenes $\left(R^{3}=H\right)$ were suitable substrates. Internal alkenes fail to deliver the product. The iminoacylation reaction can be performed with carboxylic acids in presence of $(\mathrm{Boc})_{2} \mathrm{O}$ instead of the acid chlorides or anhydrides. This procedure is an alternative to Bower's Pd catalyzed 1,2-iminoacylation of oxime esterstethered alkenes with carbon monoxide and organoborons ${ }^{96}$ (see Subsection 2.1.6.: Synthesis of 1-pyrrolines by palladium catalysis).

2.1.6. Synthesis of 1-pyrrolines by palladium catalysis. Bower et al. ${ }^{96}$ developed an extraordinary umpolung approach to obtain a great variety of 1-pyrrolines (Scheme 34 ). Their methodology is based on the oxidative addition of $\operatorname{Pd}(0)$ into the

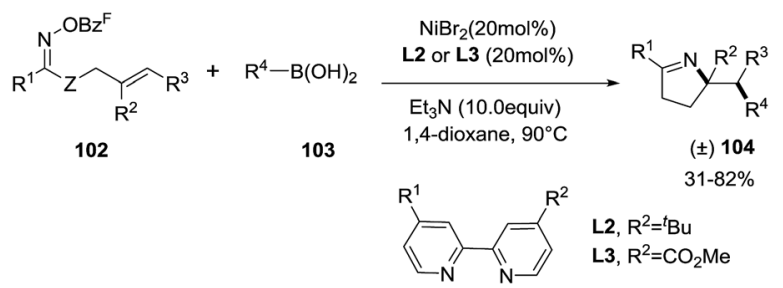

Scheme 32 Synthesis of 1-pyrrolines 104 by $\mathrm{NiBr}_{2}$.

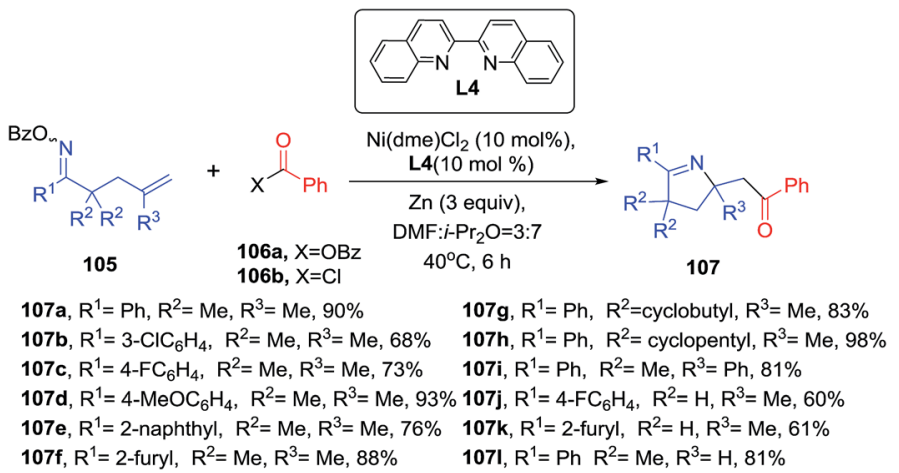

Scheme $33 \mathrm{Ni}$-catalyzed 1,2-iminoacylation of alkenes towards 1pyrrolines.

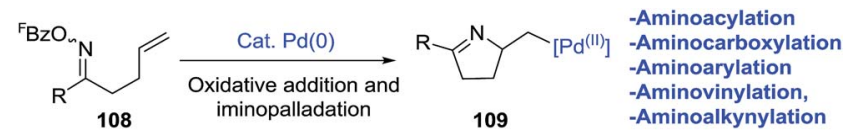

Scheme 34 Oxidative addition in the oxime ester $\mathrm{N}-\mathrm{O}$ bond

$\mathrm{N}-\mathrm{O}$ bond of $\mathrm{O}$-pentafluorobenzoyl oxime esters (108) to generate an imino-Pd(II) intermediate 109. This intermediate undergoes an 5-exo cyclization with sterically diverse alkenes to form alkyl-Pd(II) pyrroline intermediates. These intermediates can be decorated by subsequent reaction with organometallic or alcohol nucleophiles (Scheme 35). Moreover, the procedure can be carried out in presence of $\mathrm{CO}$ (carbonylative) or absence of CO (non-carbonylative) conditions. Under carbonylative conditions, 1,2-aminoacylation reactions are achieved by using triethylammonium tetraarylborates or pinacol organoboronates as the nucleophiles (products are $\mathbf{1 1 0}$ and 111). With alcohol nucleophiles under carbonylative conditions, a 1,2-aminocarboxylation occurs (product is 112).

Under noncarbonylative conditions 1,2-aminoarylation is achieved employing a variety of pinacol arylboronates as nucleophiles (Scheme 36, products 113a-j). 1,2-Aminovinylation and 1,2-aminoalkynylation products are obtained using vinyl boronates or alkynyl-stannanes, respectively (products are 113k-p). For alkynylation, less toxic pinacol

1,2-Aminoacylation Reactions: Scope of the Oxime Ester and Alkene

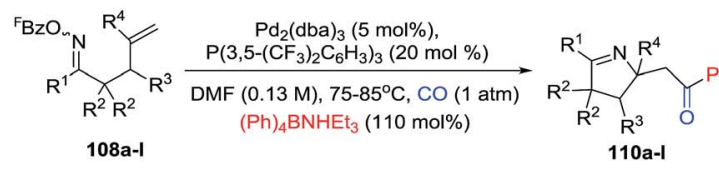

110a, $R^{1}=P h, R^{4}=M e, 62 \% \quad 110 g, R^{1}=P h, R^{4}=P h, 54 \%$

114b, $\mathrm{R}^{1}=i-\operatorname{Pr}, \mathrm{R}^{4}=\mathrm{Me}, 61 \% \quad 110 \mathrm{~h}, \mathrm{R}^{1}=\mathrm{Ph}, 42 \%$

110c, $R^{1}=c-P r, R^{4}=M e, 60 \% \quad 110 i, R^{1}=P h, R^{2}=M e, 78 \%$

110d, $R^{1}=P h, R^{4}=E t, 63 \% \quad 110 j, R^{1}=P h, R^{2}=-\left(\mathrm{CH}_{2}\right)_{5}, 72 \%$

110e, $\mathrm{R}^{1}=\mathrm{Ph}, \mathrm{R}^{4}=i-\mathrm{Pr}, 58 \% \quad 110 \mathrm{k}, \mathrm{R}^{1}=\mathrm{Ph}, \mathrm{R}^{2}=-\left(\mathrm{CH}_{2}\right)_{3^{-}}, 65 \%$

110f, $\mathrm{R}^{1}=\mathrm{Ph}, \mathrm{R}^{4}=\mathrm{Bn}, 62 \% \quad 110 \mathrm{l}, \mathrm{R}^{1}=\mathrm{Ph}, \mathrm{R}^{3}=i-\mathrm{Pr}, 61 \%$

${ }^{a} R^{2-4}=H$ unless specified otherwise

1,2-Aminoacylation Reactions: Scope of the C-Based Nucleophile
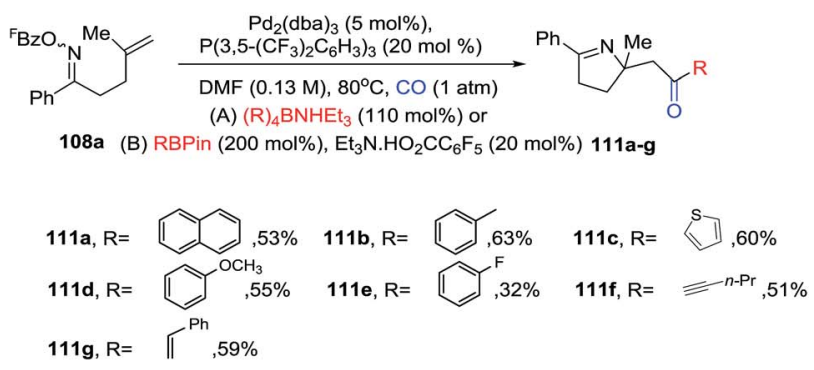

1,2-Aminocarboxylation Reactions

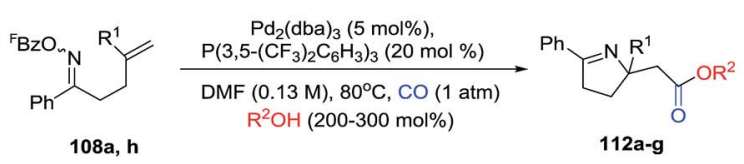

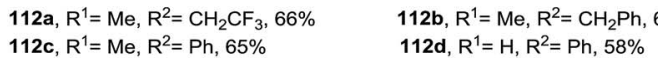

Scheme $35 \mathrm{Pd}$-catalyzed 1,2-carboamination of alkenes. 


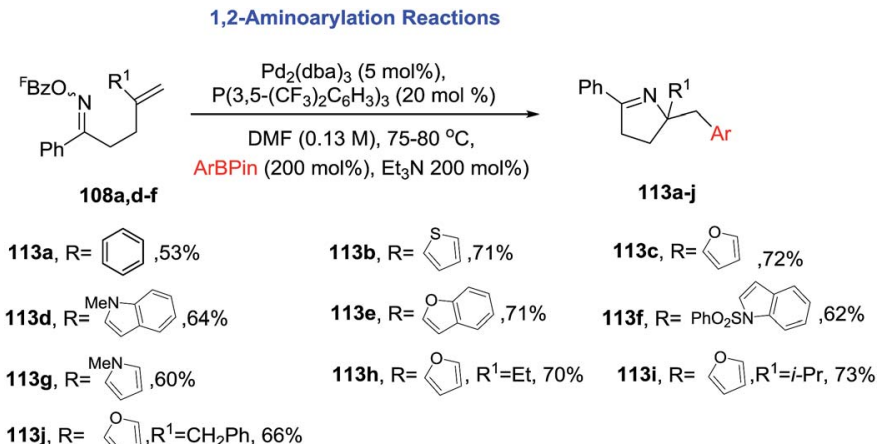

1,2-Amino-Vinylation and -Alkynylation Reactions

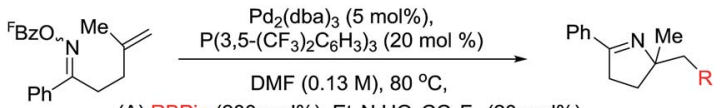

(A) RBPin (200 mol\%), $\mathrm{Et}_{3} \mathrm{~N} . \mathrm{HO}_{2} \mathrm{CC}_{6} \mathrm{~F}_{5}(20 \mathrm{~mol} \%)$

108a (B) $\mathrm{RSnBu}_{3}(200 \mathrm{~mol} \%), \mathrm{Et}_{3} \mathrm{~N}(200 \mathrm{~mol} \%)$

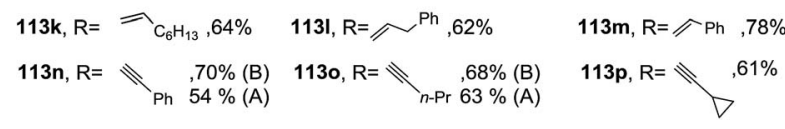

Scheme 36 Pd-catalyzed 1,2-carboamination of alkenes.

alkynylboronates can be used instead of alkynyl-stannanes. This approach provides an unified strategy for achieving alkene 1,2aminoacylation, -carboxylation, -arylation, -vinylation, and -alkynylation.

Previously Bower et al. reported ${ }^{97}$ the Pd-catalyzed cyclization of $O$-pentafluorobenzoyl oxime esters with 1,1-disubstituted alkenes as a general entry to chiral $\alpha, \alpha$-disubstituted pyrrolines (115). In this study the key ligand $\mathrm{P}\left(3,5-\left(\mathrm{CF}_{3}\right)_{2} \mathrm{C}_{6} \mathrm{H}_{3}\right)_{3}$ used in the achiral catalytic systems was replaced by the TADDOL-derived phosphoramidite L5. With $\mathbf{1 1 4}$ as the starting material, modest enantioselectivities (typically $32-37 \%$ ee) were achieved (Scheme 37). Nonetheless, these results establish the feasibility of asymmetric Narasaka-Heck cyclizations.

The same group performed Pd-catalyzed cyclizations of oxime esters with 1,2-dialkylated alkenes (116) as a general entry to chiral dihydropyrroles (117) and avoiding the formation of the undesired pyrrole (Scheme 38). ${ }^{98}$ In the previous case where 1,1-disubstituted alkenes were used, the nature of the alkene acceptor controlled the direction of $\beta$-hydride elimination and the pyrrole formation was bypassed. The selectivity in favor of the pyrroline, and against the pyrrole, derives from the

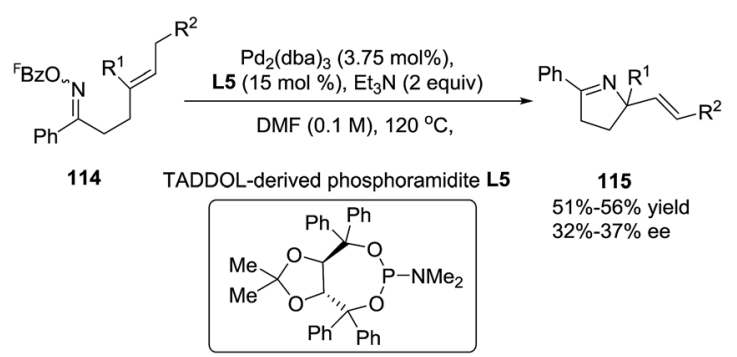

Scheme 37 Asymmetric Pd-catalyzed cyclization of oxime esters with 1,1-disubstituted alkenes.

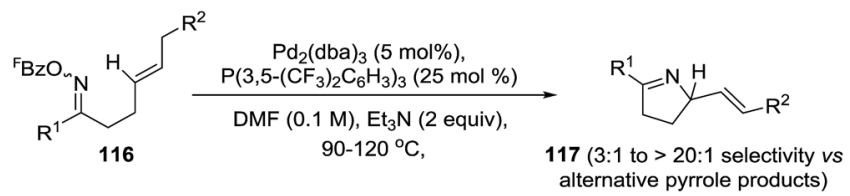

Scheme $38 \mathrm{Pd}$-catalyzed cyclization of oxime esters with 1,2-disubstituted alkenes.

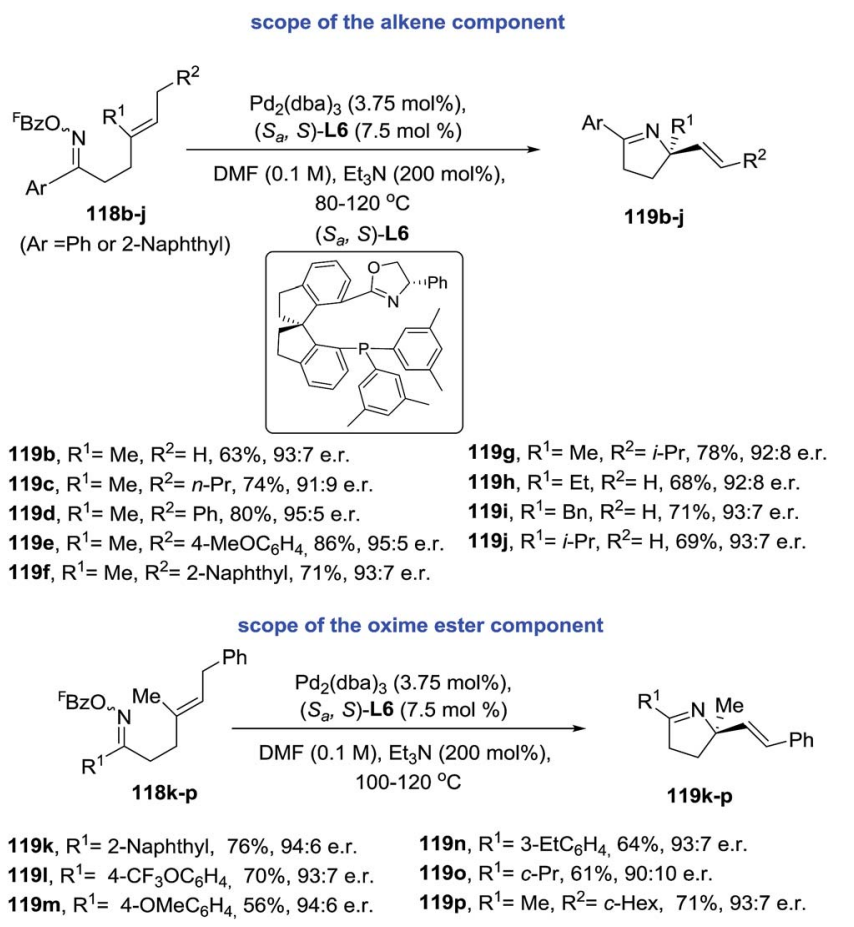

Scheme 39 Enantioselective Narasaka-Heck cyclizations.

aryl moiety $\left(\mathrm{R}^{2}=\right.$ aryl). This moiety weakens the benzylic $\mathrm{C}-\mathrm{H}$ bond and so increases the propensity for $\beta$-hydride elimination. Where $\mathrm{R}^{2} \neq$ aryl, the pyrroline/pyrrole selectivity is lower.

Very recently, Bower and coworkers ${ }^{99}$ identified a SPINOLderived P-N ligand system $\left[\left(S_{\mathrm{a}}, S\right)-\mathbf{L 6}\right]$ that promoted the first examples of highly enantioselective Narasaka-Heck cyclizations (Scheme 39). This Pd-catalyzed 5-exo cyclization tolerates a range of oxime esters and diverse sterically trisubstituted alkenes (118) and generates otherwise challenging pyrrolidine derivatives containing tetrasubstituted nitrogen-bearing stereocenters (119). Cycled products are obtained in moderate to excellent yields (56-86\%) and high enantioselectivity (91: 9 to 95 : 5 e.r.).

Almost simultaneously with the report of Bower, Bao et al. ${ }^{\mathbf{1 0 0}}$ reported a domino processes that involved a palladiumcatalyzed Narasaka-Heck reaction followed by direct arene $\mathrm{C}-\mathrm{H}$ alkylation leading to 2,5,5-trisubstituted dihydropyrroles (122) (Scheme 40). In this process two new bonds were formed: one $\mathrm{N}\left(\mathrm{sp}^{2}\right)-\mathrm{C}\left(\mathrm{sp}^{3}\right)$ bond and one $\mathrm{C}\left(\mathrm{sp}^{3}\right)-\mathrm{C}\left(\mathrm{sp}^{2}\right)$ bond and a quaternary center was generated. The optimized conditions of $\mathrm{Pd}(\mathrm{OAc})_{2}$ (0.1 equiv.), ( \pm )-BINAP (0.2 equiv.), ${ }^{\mathrm{i}} \mathrm{Pr}_{2} \mathrm{NEt}$ (4.0 equiv.), $\mathrm{Cs}_{2} \mathrm{CO}_{3}$ (3.0 equiv.) in DMSO at $120{ }^{\circ} \mathrm{C}$ were applied to substrates with a range of substituted on the aryl (Ar) group of 


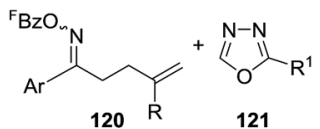

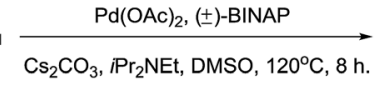
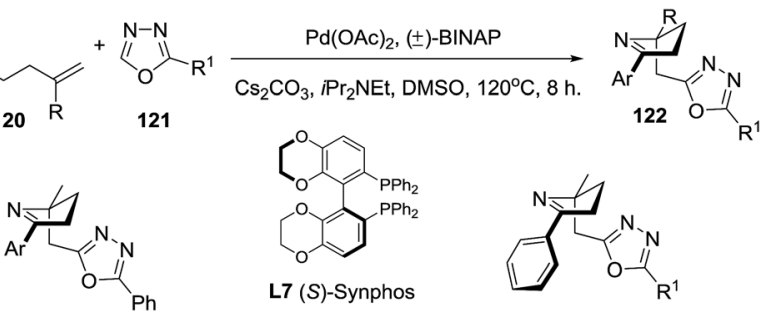

122aa, $\mathrm{Ar}=\mathrm{Ph}, 90 \%$

122ba, $\mathrm{Ar}=2-\mathrm{MeC}_{6} \mathrm{H}_{4}, 86 \%$

122ca, $\mathrm{Ar}=3-\mathrm{MeC}_{6} \mathrm{H}_{4}, 92 \%$

122da, $\mathrm{Ar}=4-\mathrm{MeC}_{6} \mathrm{H}_{4}, 89 \%$

122ea, $\mathrm{Ar}=3,4-\mathrm{Me}_{2} \mathrm{C}_{6} \mathrm{H}_{3}, 73 \%$

122fa, $\mathrm{Ar}=4-\mathrm{MeOC}_{6} \mathrm{H}_{4}, 81 \%$

122ga, $\mathrm{Ar}=4-\mathrm{ClC}_{6} \mathrm{H}_{4}, 84 \%$

122ha, $\mathrm{Ar}=4-\mathrm{CF}_{3} \mathrm{OC}_{6} \mathrm{H}_{4}, 69 \%$

122ia, $\mathrm{Ar}=4-\mathrm{FC}_{6} \mathrm{H}_{4}, 46 \%$

122ja, Ar= 2-Naphthyl, $80 \%$
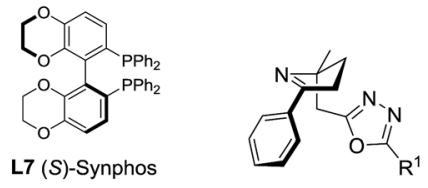

122ab, $\mathrm{R}^{1}=4-\mathrm{PhC}_{6} \mathrm{H}_{4}, 67 \%$

122ac, $\mathrm{R}^{1}=4-\mathrm{CF}_{3} \mathrm{OC}_{6} \mathrm{H}_{4}, 71 \%$

122ad, $\mathrm{R}^{1}=4-\mathrm{MeC}_{6} \mathrm{H}_{4}, 81 \%$

122ae, $R^{1}=4-\mathrm{BuC}_{6} \mathrm{H}_{4}, 69 \%$

122af, $\mathrm{R}^{1}=3-\mathrm{MeOC}_{6} \mathrm{H}_{4}, 73 \%$

122ag, $R^{1}=2-$ Naphthyl, $58 \%$

122ah, $\mathrm{R}^{1}=n \mathrm{Bu}, 42 \%$

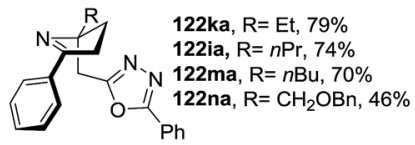

Scheme 40 Domino Narasaka-Heck reaction/direct arene $\mathrm{C}-\mathrm{H}$ alkylation.

the oxime ester (120), exploring both the electronic nature and position of the substituent. In most of cases, the 1-pyrrolines 122aa-122ja were obtained in very good yields. A variety of substituents on the oxadiazole (121), and different alkyl groups attached to the alkene, were well tolerated. An enantioselective version of this cascade process was developed using (S)-Synphos (L7) as chiral ligand, isolating the 2,5,5-trisubstituted pyrroline 122 in good yields with good to high enantioselectivity.

The Glorius group ${ }^{101}$ developed an entry to 1-pyrrolines $(\mathbf{1 2 4}$, $\mathbf{1 2 4}^{\prime}, \mathbf{1 2 4}^{\prime \prime}$ ) starting from the imines 123 derived from acetophenone and cyclic $N$-allylamines (Scheme 41 ). The procedure employs a mild oxidative palladium-catalyzed cyclization using $\mathrm{O}_{2}$ as the terminal oxidant. Besides cyclic $\mathrm{N}$-allylamines, $\mathrm{N}$ allylamines containing $\gamma$-substituents on the allyl group also deliver the 1-pyrrolines in good yields (61-87\%). This atomeconomical procedure relies on an intramolecular $\mathrm{C}-\mathrm{H}$ dehydrogenative 5-exo cyclization.

Tong et al. ${ }^{102}$ developed a methodology to synthesize 2-halomethyl 1-pyrrolines 126 from the oxime ester 125 using a $\operatorname{Pd}(0)$ catalyzed intramolecular iminohalogenations of 1,1-disubstituted alkenes assisted with halide salts (Scheme 42). The use of electron-poor phosphine as ligands proved to be essential to obtain alkyl halogenated dihydropyrroles under reductive elimination. Iminochlorination (128) is less efficient compared to iminobromination (127) and iminoiodination (126). For example, when $\mathrm{R}^{1}=\mathrm{Ph}$ and $\mathrm{R}^{2}=$ Me the yields for the iminohalogenation are $75 \%$ for iodination, $64 \%$ for bromination and $42 \%$ for chlorination.

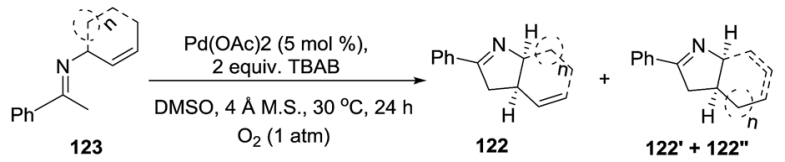

Scheme 41 Palladium(॥)-catalyzed cyclization of imines to 1pyrrolines.

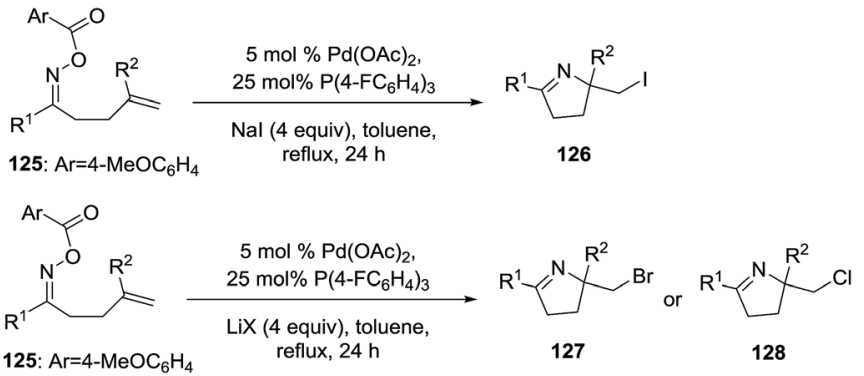

Scheme 42 Palladium(0)-catalyzed iminohalogenation of alkenes.

Homoallylic primary amines $\mathbf{1 2 9}$ reacted with aryl iodides 130 in the presence of a Pd catalyst to provide 2-aryl-1-pyrrolines 132 via a cascade $\mathrm{C}-\mathrm{H}$ arylation/ $\mathrm{C}-\mathrm{H}$ amination sequence (Scheme 43). ${ }^{103}$ The transformation proceeds thorough a Pdpromoted Heck coupling followed by a $\mathrm{C}-\mathrm{H}$ amination cocatalyzed by Pd and the aryl iodide, and final tautomarization from 2-pyrrolines to the isolated 1-pyrrolines. Interestingly, this report represents the first example of aryl iodide acting as both coupling reagent and cocatalyst involved in the generation of the active bivalent aryl palladium catalyst $\mathbf{1 3 1}$ capable of promoting the formation of new $\mathrm{N}-\mathrm{C}$ bond. The optimized protocol involved the use of 2 eq. of aryl iodide 130, $\mathrm{Pd}(\mathrm{OAc})_{2}$ (10 mol\%), MBQ (2-methylbenzoquinone, $20 \mathrm{~mol} \%$ ) as oxidant in DMF at $80{ }^{\circ} \mathrm{C}$, leading to a small collection of the final heterocycles 132 with poor to good yields (26-74\%). The presence of an oxidant was essential to achieve complete annulation step.

2.1.7. Synthesis of 1-pyrrolines by rhodium catalysis. The hydroamination of alkynes is a highly atom-efficient approach to the synthesis of imines and consist of the $\mathrm{N}-\mathrm{H}$ addition to a C-C triple bond. If the reaction is intramolecular, the results are cycled imines as 1-pyrrolines. Late transition metal complexes of $\mathrm{Ru}, \mathrm{Rh}, \mathrm{Ir}, \mathrm{Pd}, \mathrm{Pt}$, and $\mathrm{Cu}$ are effective catalysts for the hydroamination reaction. ${ }^{\mathbf{1 0 4 , 1 0 5}}$

In 2012, Messerle and coworkers ${ }^{87}$ reported a series of cationic rhodium $(\mathbf{1 3 3}, \mathbf{1 3 5})$ and iridium complexes $(\mathbf{1 3 4}, \mathbf{1 3 6})$ with bidentate $N, N^{\prime}$-pyrazolyl-triazolyl ligands (Fig. 3 ). These complexes were evaluated as catalysts for the intramolecular hydroamination of a series of alkynylamines. In the case of the cyclization of the terminal alkyne as 4-pentyn-1-amine (137) to 2-methyl-1-pyrroline (138) at $60{ }^{\circ} \mathrm{C}$ (Scheme 44), the iridium complexes $\left[\operatorname{Ir}(\mathrm{N}-\mathrm{N})(\mathrm{CO})_{2}\right]-\mathrm{BArF}_{4}(\mathbf{1 3 6 c}, \mathbf{d})$ were the most active catalysts giving 5-exo-cyclization exclusively (>98\% yield).

The rhodium complexes generally were less active as catalysts than their iridium analogues. In the case of intermolecular hydroamination of internal alkynes, such as with 4phenyl-3-butyn-1-amine (139a) and 5-phenyl-4-pentyn-1amine (139b), the reaction required a higher temperature $\left(110{ }^{\circ} \mathrm{C}\right)$ to reach complete conversion $(>98 \%)$ towards pyrrolines 140a,b (Scheme 45).

Rhodium(I) catalysts with ligands such as $\left(\mathrm{Cy}_{2} \mathrm{P}\right)_{2} \mathrm{NMe}$ and $\operatorname{PNP}(\mathrm{Cy})$ are efficient reagents to perform the union of Schelating aldehydes 141 (aldehydes with a methyl sulfide group in $\beta$ position) and allylic amines $\mathbf{1 4 2}$ to deliver linear 

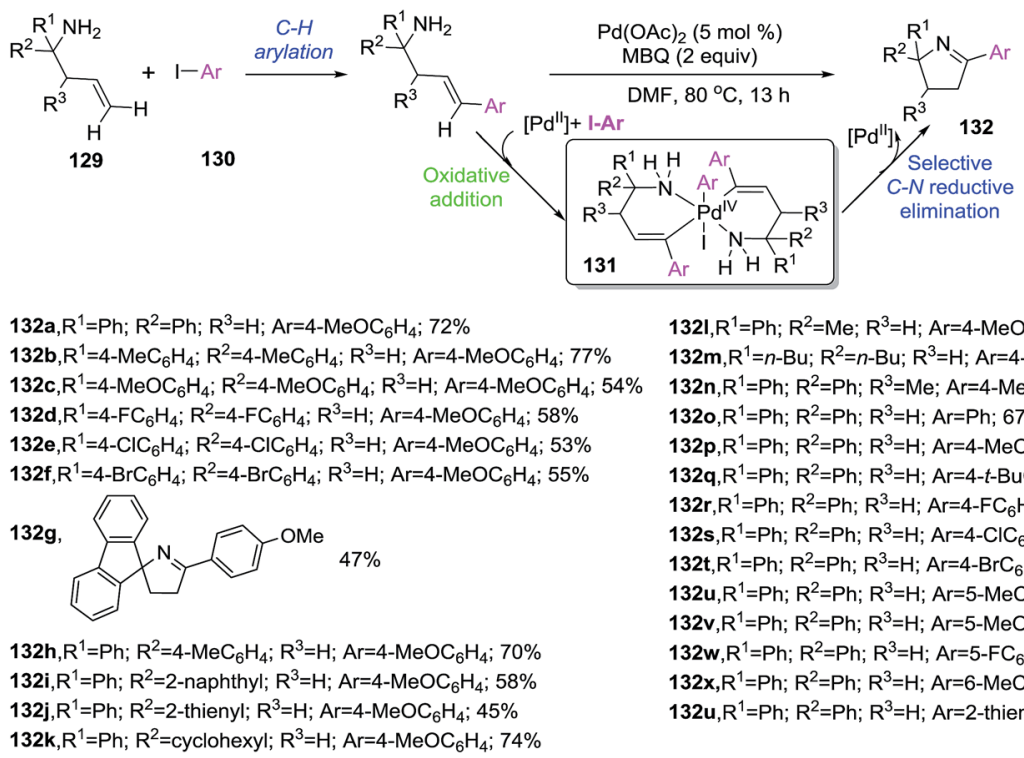

132I, $R^{1}=\mathrm{Ph} ; \mathrm{R}^{2}=\mathrm{Me} ; \mathrm{R}^{3}=\mathrm{H} ; \mathrm{Ar}=4-\mathrm{MeOC}_{6} \mathrm{H}_{4} ; 26 \%$

$132 \mathrm{~m}, \mathrm{R}^{1}=n-\mathrm{Bu} ; \mathrm{R}^{2}=n-\mathrm{Bu} ; \mathrm{R}^{3}=\mathrm{H} ; \mathrm{Ar}=4-\mathrm{MeOC}_{6} \mathrm{H}_{4}$; traces

$132 n, R^{1}=\mathrm{Ph} ; \mathrm{R}^{2}=\mathrm{Ph} ; \mathrm{R}^{3}=\mathrm{Me} ; \mathrm{Ar}=4-\mathrm{MeOC}_{6} \mathrm{H}_{4} ; 67 \%$

132o, $\mathrm{R}^{1}=\mathrm{Ph} ; \mathrm{R}^{2}=\mathrm{Ph} ; \mathrm{R}^{3}=\mathrm{H} ; \mathrm{Ar}=\mathrm{Ph} ; 67 \%$

132p, $R^{1}=\mathrm{Ph} ; \mathrm{R}^{2}=\mathrm{Ph} ; \mathrm{R}^{3}=\mathrm{H} ; \mathrm{Ar}=4-\mathrm{MeC}_{6} \mathrm{H}_{4} ; 58 \%$

132q, $R^{1}=P h ; R^{2}=P h ; R^{3}=H ; A r=4-t-B^{2} C_{6} H_{4} ; 62 \%$

$132 r, R^{1}=\mathrm{Ph} ; \mathrm{R}^{2}=\mathrm{Ph} ; \mathrm{R}^{3}=\mathrm{H} ; \mathrm{Ar}=4-\mathrm{FC}_{6} \mathrm{H}_{4} ; 65 \%$

132s, $R^{1}=\mathrm{Ph} ; \mathrm{R}^{2}=\mathrm{Ph} ; \mathrm{R}^{3}=\mathrm{H} ; \mathrm{Ar}=4-\mathrm{ClC}_{6} \mathrm{H}_{4} ; 52 \%$

132t, $\mathrm{R}^{1}=\mathrm{Ph} ; \mathrm{R}^{2}=\mathrm{Ph} ; \mathrm{R}^{3}=\mathrm{H} ; \mathrm{Ar}=4-\mathrm{BrC}_{6} \mathrm{H}_{4} ; 50 \%$

$132 u, R^{1}=P h ; R^{2}=P h ; R^{3}=H ; A r=5-M e C_{6} \mathrm{H}_{4} ; 55 \%$

$132 v, R^{1}=\mathrm{Ph} ; \mathrm{R}^{2}=\mathrm{Ph} ; \mathrm{R}^{3}=\mathrm{H} ; \mathrm{Ar}=5-\mathrm{MeOC}_{6} \mathrm{H}_{4} ; 63 \%$

132w, $\mathrm{R}^{1}=\mathrm{Ph} ; \mathrm{R}^{2}=\mathrm{Ph} ; \mathrm{R}^{3}=\mathrm{H} ; \mathrm{Ar}=5-\mathrm{FC}_{6} \mathrm{H}_{4} ; 55 \%$

$132 x, R^{1}=P h ; R^{2}=P h ; R^{3}=H ; A r=6-M_{e} C_{6} H_{4} ; 70 \%$

132u, $\mathrm{R}^{1}=\mathrm{Ph} ; \mathrm{R}^{2}=\mathrm{Ph} ; \mathrm{R}^{3}=\mathrm{H} ; \mathrm{Ar}=2$-thienyl; $46 \%$

Scheme 43 Synthesis of 2-aryl-1-pyrrolines 132 via a cascade $\mathrm{C}-\mathrm{H}$ arylation/ $\mathrm{C}-\mathrm{H}$ amination sequence.

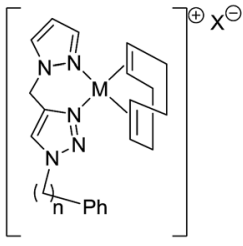

$\mathrm{Rh}, 133 \mathrm{a} ; \mathrm{Ir}, 134 \mathrm{a}$ Rh, 133b; Ir, 134b Rh, 133c; Ir, 134c

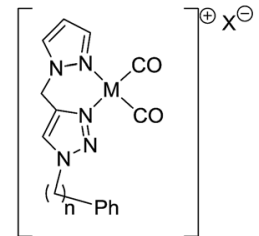

Rh, 135a; Ir, 136a $\quad \mathrm{n}=1, \mathrm{X}=\mathrm{BPh}$

$\mathrm{Rh}, 135 \mathrm{~b} ; \mathrm{Ir}, 136 \mathrm{~b} \quad \mathrm{n}=0, \mathrm{X}=\mathrm{BPh}_{4}$

Rh, 135c; Ir, 136c $\quad \mathrm{n}=1, \mathrm{X}=\mathrm{BAr}_{4}$

$\mathrm{Rh}, 135 \mathrm{~d}$; Ir, 136d $\mathrm{n}=0, \mathrm{X}=\mathrm{BAr}_{4}$

Fig. 3 Pyrazolyl-triazolyl bidentate ligands and their rhodium(I) and iridium(ı) complexes.

hydroacylation adducts 143. These adducts are treated in situ with $p$-TsOH to provide the pyrrolines 144 through a dehydrative cyclization (Scheme 46). ${ }^{23}$ The pyrroline products $(\mathbf{1 4 4})$ are formed in good-to-excellent yields (48-90\%).

A variety of alkyl, aryl and heteroaryl substituents $\left(\mathrm{R}^{2}=\mathrm{Ar}\right.$, HetAr, alkyl) were well tolerated at the allylic position of the alkenes. Also, different aryl or heteroaryl $\left(\mathrm{R}^{1}=\mathrm{Ar}\right.$, HetAr) Schelated aldehydes provided the pyrrolines. Aldehydes featuring a chelating group in $\beta$-position other than methylthio

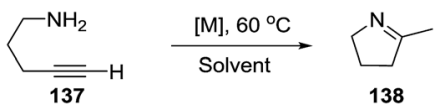

Scheme 44 Catalyzed cyclization of 4-pentyn-1-amine.

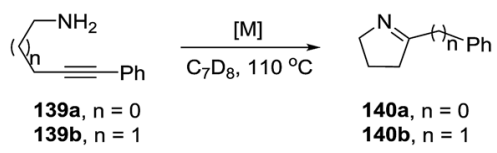

Scheme 45 Catalyzed cyclization of non-terminal alkynamines.

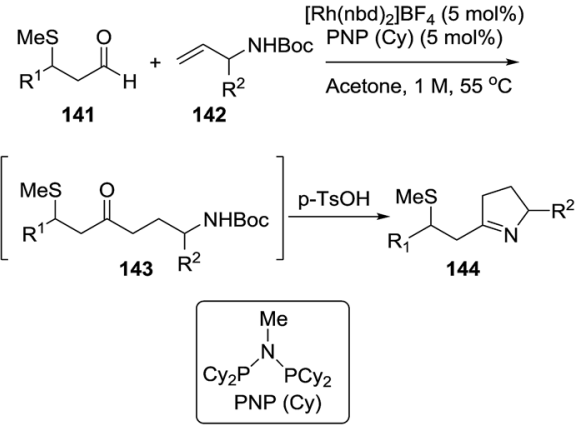

Scheme 46 Pyrroline synthesis from the combination of S-chelating aldehydes and allylic amines.

and disubstituted alkenes were not reactive under these conditions.

The Meggers group reported for first time a visible-lightinduced [2 +3 ] photocycloaddition of alkenes 145 with vinyl azides 146 using a chiral rhodium catalyst 147 to obtain enantiomeric pure 1-pyrrolines 148 (Scheme 47). ${ }^{106}$ The protocol makes use of $\alpha, \beta$-unsaturated $N$-acylpyrazole 145 in combination with vinyl azides 146 in presence of catalyst $(\Lambda-\mathrm{RhS}) 147$ (4 mol\%) using chloroform as solvent under irradiation of blue LEDs. As a result, 1-pyrrolines 148 were obtained in good to excellent yields (69-94\%) with complete diastereoselectivity and enantioselectivities of up to $>99 \%$ ee. The pyrazole auxiliary have a major impact on the reaction result, the best performance is achieved with 3-( $p$-methoxyphenyl)pyrazole (PMP). The chiral rhodium catalyst 147, developed in Megger's group, coordinates with substrates 145 through the carbonyl oxygen and the pyrazole auxiliary. This complex I generates an excited intermediate $\mathbf{I}^{*}$ upon visible-light irradiation which subsequently reacts with a vinyl azides to afford a 1-pyrrolines (Scheme 47). The methodology tolerates various $\beta$-aryl 


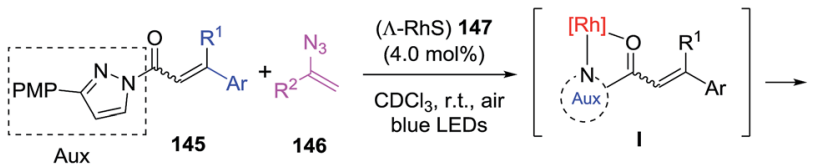

Visible light
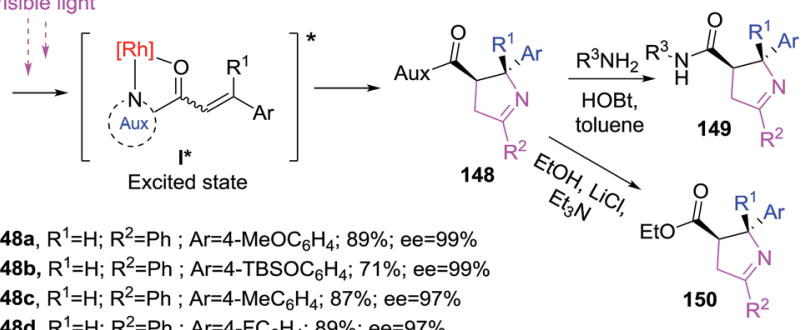

148a, $\mathrm{R}^{1}=\mathrm{H} ; \mathrm{R}^{2}=\mathrm{Ph} ; \mathrm{Ar}=4-\mathrm{MeOC}_{6} \mathrm{H}_{4} ; 89 \% ;$ ee $=99 \%$ 148b, $\mathrm{R}^{1}=\mathrm{H} ; \mathrm{R}^{2}=\mathrm{Ph} ; \mathrm{Ar}=4-\mathrm{TBSOC}_{6} \mathrm{H}_{4} ; 71 \%$; ee=99\% 148c, $\mathrm{R}^{1}=\mathrm{H} ; \mathrm{R}^{2}=\mathrm{Ph} ; \mathrm{Ar}=4-\mathrm{MeC}_{6} \mathrm{H}_{4} ; 87 \% ;$ ee=97\% 148d, $R^{1}=H ; R^{2}=P h ; A r=4-F_{6} H_{4} ; 89 \% ;$ ee $=97 \%$ 148e, $\mathrm{R}^{1}=\mathrm{H} ; \mathrm{R}^{2}=\mathrm{Ph} ; \mathrm{Ar}=4-\mathrm{BrC}_{6} \mathrm{H}_{4} ; 87 \% ;$ ee $=97 \%$ 148f, $\mathrm{R}^{1}=\mathrm{H} ; \mathrm{R}^{2}=\mathrm{Ph} ; \mathrm{Ar}=4-\mathrm{CF}_{3} \mathrm{C}_{6} \mathrm{H}_{4} ; 90 \%$; ee=95\% 148g, $\mathrm{R}^{1}=\mathrm{H} ; \mathrm{R}^{2}=\mathrm{Ph} ; \mathrm{Ar}=3-\mathrm{MeOC}_{6} \mathrm{H}_{4} ; 84 \%$; ee $=98 \%$ 148h, $\mathrm{R}^{1}=\mathrm{H} ; \mathrm{R}^{2}=\mathrm{Ph} ; \mathrm{Ar}=3-\mathrm{MeOC}_{6} \mathrm{H}_{4} ; 94 \%$; ee $=94 \%$ 148i, $\mathrm{R}^{1}=\mathrm{H} ; \mathrm{R}^{2}=\mathrm{Ph} ; \mathrm{Ar}=2$-thienyl; $77 \%$; ee $=90 \%$

$148 \mathrm{j}, \mathrm{R}^{1}=\mathrm{H} ; \mathrm{R}^{2}=\mathrm{Ph} ; \mathrm{Ar}=$

148k, $R^{1}=\mathrm{Me} ; \mathrm{R}^{2}=\mathrm{Ph} ;$ Ar=4-MeOC $6 \mathrm{H}_{4} ; 91 \%$; ee=98\% (from $(E)$ ); $89 \%$, ee $=98 \%$ (from $(Z)$ )

148I, $R^{1}=H ; R^{2}=P h ; A r=3,5-d i C{ }_{3} C_{6} H_{4} ; 50 \% ;$ ee $=97 \%$

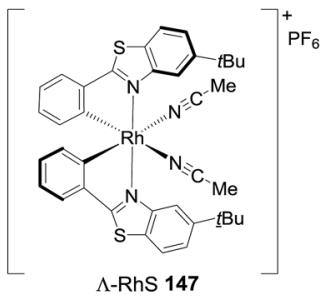

148m,

148n, $R^{1}=H ; R^{2}=4-M_{e} C_{6} H_{4} ; \mathrm{Ar}=\mathrm{Ph} ; 75 \% ;$ ee $=90 \%$ 148o, $R^{1}=\mathrm{H} ; \mathrm{R}^{2}=4-\mathrm{ClC}_{6} \mathrm{H}_{4} ; \mathrm{Ar}=\mathrm{Ph} ; 80 \%$; ee $=95 \%$ 148p, $\mathrm{R}^{1}=\mathrm{H} ; \mathrm{R}^{2}=3-\mathrm{ClC}_{6} \mathrm{H}_{4} ; \mathrm{Ar}=\mathrm{Ph} ; 92 \%$; ee $=95 \%$ 148q, $\mathrm{R}^{1}=\mathrm{H} ; \mathrm{R}^{2}=2-\mathrm{ClC}_{6} \mathrm{H}_{4} ; \mathrm{Ar}=\mathrm{Ph} ; 80 \%$; ee $=98 \%$ $148 r, R^{1}=H ; R^{2}=2-B r C_{6} H_{4} ; A r=P h ; 89 \% ; e e=98 \%$ 148s, Aux Ph $75 \%$; ee=93\%

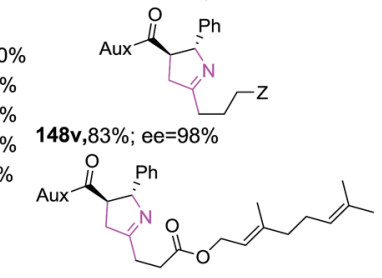

148t, $Z=n \operatorname{Pr} 70 \%$; ee $=97 \%$ $148 \mathrm{u}, \mathrm{Z}=\mathrm{OH} 69 \%$; ee $=98 \%$

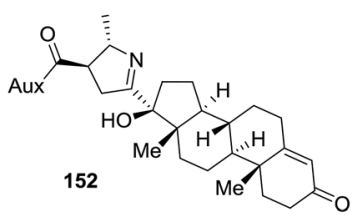

$89 \%$ yield; >20:1d.r.

Ethisterone derivative 151

Scheme 47 Synthesis of 1-pyrrolines using a chiral rhodium catalyst.

substituent on $\mathrm{N}$-acylpyrazole 145 regardless the electronic nature or position as well as heteroaryl moieties and is able to provide 1-pyrrolines with quaternary stereocenter $(\mathrm{R} \neq \mathrm{H}) . \alpha, \beta$ Unsaturated $N$-acylpyrazoles with $E$ or $Z$ configuration produce the same result. Aryl, vinyl and alkyl azides 146 successfully afforded the desired 1-pyrrolines. The good functional group tolerance was tested with a complex steroid vinyl azide derivative $\mathbf{1 5 1}$ proving the utility of the protocol in late stage synthesis. Finally, the $N$-acylpyrazole can be easily converted in other functionalities such as amide 149 or ester 150.

Katukojvala et al. discovered a new rhodium-catalyzed [1+1 +3] annulation reaction of diazoenals 153 and vinyl azides 154 to generate enal-functionalized 1-pyrroline derivatives $\mathbf{1 5 5}$

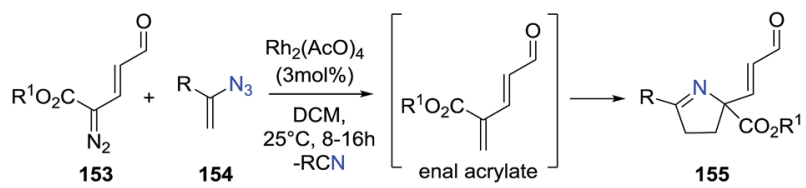

155a, R=Ph; $\mathrm{R}^{1}=\mathrm{Et} ; 71 \% ; 10 \mathrm{~h}$ 155b, R=Ph; $\mathrm{R}^{1}=\mathrm{Bn} ; 80 \% ; 8 \mathrm{~h}$ 155c, $\mathrm{R}=\mathrm{Ph} ; \mathrm{R}^{1}=\mathrm{Me} ; 66 \% ; 10 \mathrm{~h}$ 155d, R=Ph; $\mathrm{R}^{1}=t \mathrm{Bu} ; 52 \% ; 12 \mathrm{~h}$ 155e, $\mathrm{R}=\mathrm{Ph} ; \mathrm{R}^{1}=$ menthyl; $72 \% ; 2: 1 \mathrm{dr} ; 8 \mathrm{~h}$ 155f, $\mathrm{R}=2-\mathrm{MeC}_{6} \mathrm{H}_{4} ; \mathrm{R}^{1}=\mathrm{Et} ; 0 \% ; 10 \mathrm{~h}$ 155g, R=3- $\mathrm{MeC}_{6} \mathrm{H}_{4} ; \mathrm{R}^{1}=\mathrm{Bn} ; 64 \% ; 8 \mathrm{~h}$ 155h, $\mathrm{R}=4-\mathrm{MeC}_{6} \mathrm{H}_{4} ; \mathrm{R}^{1}=\mathrm{Et} ; 76 \% ; 10 \mathrm{~h}$ 155i, $\mathrm{R}=2-\mathrm{MeOC}_{6} \mathrm{H}_{4} ; \mathrm{R}^{1}=\mathrm{Bn} ; 55 \% ; 12 \mathrm{~h}$ 155j, R=4- $\mathrm{MeOC}_{6} \mathrm{H}_{4} ; \mathrm{R}^{1}=\mathrm{Bn} ; 81 \% ; 8 \mathrm{~h}$ 155k, R=3,4- $\mathrm{MeOC}_{6} \mathrm{H}_{4} ; \mathrm{R}^{1}=\mathrm{Bn} ; 73 \% ; 10 \mathrm{~h}$ 155I, $\mathrm{R}=3-\mathrm{FC}_{6} \mathrm{H}_{4} ; \mathrm{R}^{1}=\mathrm{Bn} ; 58 \% ; 12 \mathrm{~h}$ 155m, R=3-CIC $\mathrm{H}_{4} ; \mathrm{R}^{1}=\mathrm{Et} ; 64 \% ; 15 \mathrm{~h}$ 155n, $\mathrm{R}=3-\mathrm{BrC}_{6} \mathrm{H}_{4} ; \mathrm{R}^{1}={ }^{t} \mathrm{Bu} ; 50 \% ; 12 \mathrm{~h}$

155o, $\mathrm{R}=4-\mathrm{ClC}_{6} \mathrm{H}_{4} ; \mathrm{R}^{1}=\mathrm{Bn} ; 67 \% ; 12 \mathrm{~h}$ 155p, $\mathrm{R}=4-\mathrm{BrC}_{6} \mathrm{H}_{4} ; \mathrm{R}^{1}=\mathrm{Me} ; 62 \% ; 16 \mathrm{~h}$ 155q, R=4- $-\mathrm{CF}_{3} \mathrm{C}_{6} \mathrm{H}_{4} ; \mathrm{R}^{1}=\mathrm{Bn} ; 35 \% ; 16 \mathrm{~h}$ $155 r, \mathrm{R}=3-\mathrm{NO}_{2} \mathrm{C}_{6} \mathrm{H}_{4} ; \mathrm{R}^{1}=\mathrm{Et} ; 46 \% ; 16 \mathrm{~h}$ 155s, $\mathrm{R}=3-\mathrm{NO}_{2} \mathrm{C}_{6} \mathrm{H}_{4} ; \mathrm{R}^{1}=\mathrm{Bn} ; 54 \% ; 16 \mathrm{~h}$ 155t, $\mathrm{R}=4-\mathrm{NO}_{2} \mathrm{C}_{6} \mathrm{H}_{4} ; \mathrm{R}^{1}=\mathrm{Et} ; 48 \% ; 16 \mathrm{~h}$ 155u, $\mathrm{R}=4-\mathrm{CNC}_{6} \mathrm{H}_{4} ; \mathrm{R}^{1}=\mathrm{Bn} ; 51 \% ; 16 \mathrm{~h}$ 155v, R=4-MeCOC $6 \mathrm{H}_{4} ; \mathrm{R}^{1}=\mathrm{Et} ; 52 \% ; 12 \mathrm{~h}$ 155w, R=2-naphthyl; $\mathrm{R}^{1}=\mathrm{Et} ; 65 \%$; $12 \mathrm{~h}$ 155x,R=2-thienyl; $\mathrm{R}^{1}=\mathrm{Et} ; 45 \% ; 10 \mathrm{~h}$ 155y, $R=2$-thienyl; $R^{1}=B n ; 57 \% ; 10 h$ 155z, R=3-thienyl; $\mathrm{R}^{1}=\mathrm{Bn} ; 62 \% ; 12 \mathrm{~h}$ 155ab, R= $\mathrm{CH}_{3}\left(\mathrm{CH}_{2}\right)_{3} ; \mathrm{R}^{1}=\mathrm{Et} ; 0 \% ; 10 \mathrm{~h}$

Scheme 48 Rhodium-catalyzed [1 + $1+3]$ annulation reaction to generate enal-functionalized 1-pyrrolines 155.

(Scheme 48). ${ }^{107}$ These functionalized 1-pyrrolines can be synthetically modified to provide further structural diversity. The reaction occurs through a rhodium-catalyzed olefination of diazoenals with vinyl azides to produce the enal acrylate and subsequent annulation to access to 1-pyrrolines. The optimized conditions involve the use of $\mathrm{Rh}_{2}(\mathrm{OAc})_{4}$ as the catalyst, and of 2.5 equiv. of vinyl azides in dichloromethane at room temperature. Ethyl, benzyl, and methyl ester diazoenals 153 delivered the 1-pyrrolines in very good yields (66-80\%). Regarding vinyl azides 154, neutral and electron-rich styryl azides afforded very good yields of functionalized 1-pyrrolines (64-81\%) but the used of electron-deficient styryl azides resulted in lower yields (35-54\%). Thienyl azides were also compatible with the reactions conditions but aliphatic vinyl azide failed to deliver the 1pyrrolines 155.

2.1.8. Synthesis of 1-pyrrolines by silver catalysis. Bai et $a .^{22}$ reported a one-pot asymmetric Michael-type conjugate

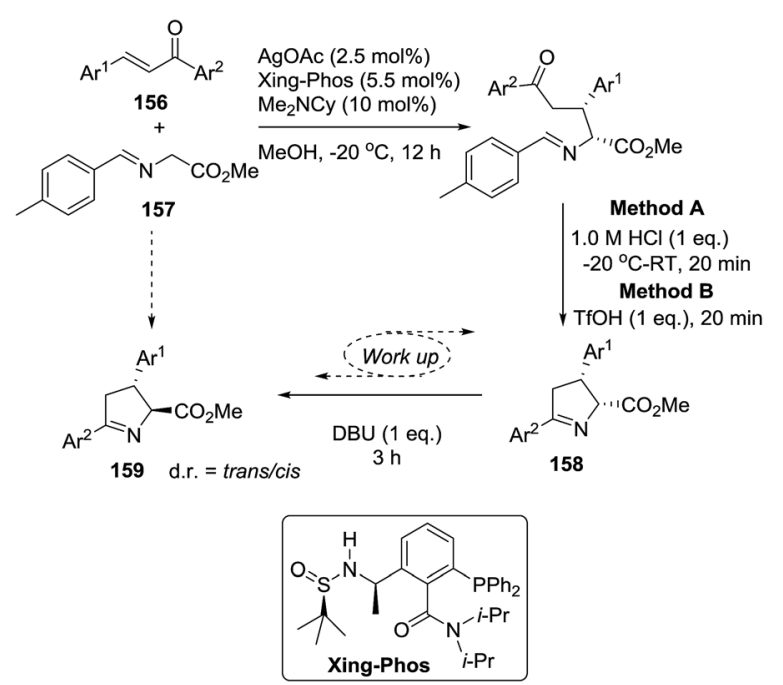

Scheme 49 Stereodivergent synthesis of cis- and trans-1-pyrrolines (158 and 159). 


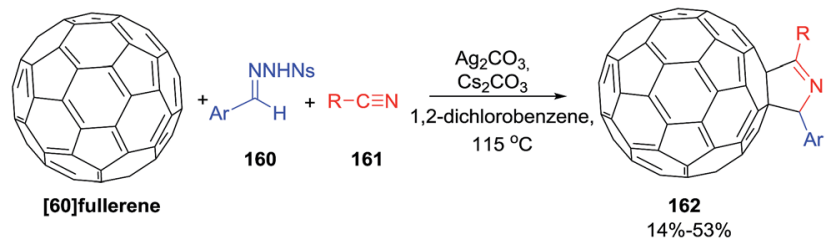

Scheme 50 Silver(I)-mediated three-component annulation reaction for the synthesis of [60]fullerene-fused 1-pyrrolines.

addition of glycine imino esters (157) to chalcones (156) using a Ag/Xing-Phos-catalyzed [3 +2$]$ cycloaddition reaction to afford stereoselectively the 1-pyrrolines 158 (Method A, Scheme 49). The combination of $\mathrm{Ag} / \mathrm{Xing}$-Phos catalyst with $\mathrm{Me}_{2} \mathrm{NCy}$ as a base gave the best performance in term of yield (81-98\%), diastereoselectivity, and enantioselectivity (up to $98 \%$ ee).

Moreover, trans-pyrrolines could be obtained using the same catalytic system but incorporating an epimerization protocol into the work-up conditions. Method A $(\mathrm{HCl})$ gave the cis-pyrrolines 158 whereas the use of TfOH and DBU (Method B) led to the trans-pyrrolines 159 which were obtained with yields
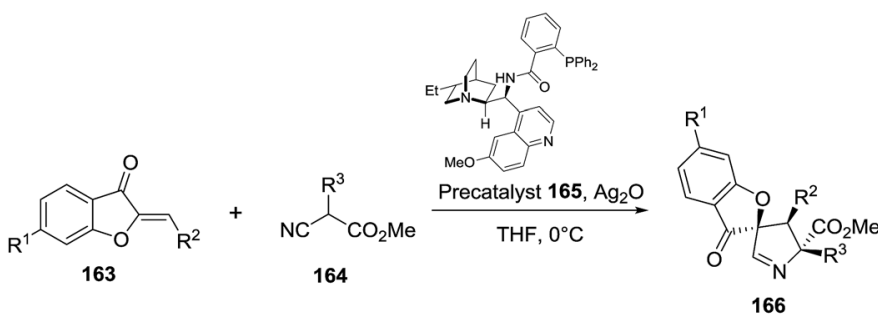

166a, $\mathrm{R}^{1}=\mathrm{H} ; \mathrm{R}^{2}=\mathrm{Ph} ; \mathrm{R}^{3}=\mathrm{Bn} ; 99 \%,>20: 1 \mathrm{dr}, 93 \%$ ee 166b, $R^{1}=\mathrm{OMe} ; \mathrm{R}^{2}=\mathrm{Ph} ; \mathrm{R}^{3}=\mathrm{Bn} ; 99 \%,>20: 1 \mathrm{dr}, 93 \%$ ee 166c, $R^{1}=B r ; R^{2}=P h ; R^{3}=B n ; 95 \%,>20: 1 d r, 94 \%$ ee 166d, $R^{1}=H ; R^{2}=2-\mathrm{ClC}_{6} \mathrm{H}_{4} ; \mathrm{R}^{3}=\mathrm{Bn} ; 99 \%, 20: 1 \mathrm{dr}, 97 \%$ ee 166e, $R^{1}=H ; R^{2}=2-M_{e C} H_{4} ; R^{3}=B n ; 99 \%, 20: 1 \mathrm{dr}, 94 \%$ ee 166f, $\mathrm{R}^{1}=\mathrm{H} ; \mathrm{R}^{2}=3-\mathrm{ClC}_{6} \mathrm{H}_{4} ; \mathrm{R}^{3}=\mathrm{Bn} ; 98 \%, 18: 1 \mathrm{dr}, 88 \%$ ee 166g, $\mathrm{R}^{1}=\mathrm{H} ; \mathrm{R}^{2}=3-\mathrm{MeOC}_{6} \mathrm{H}_{4} ; \mathrm{R}^{3}=\mathrm{Bn} ; 98 \%,>20: 1 \mathrm{dr}, 93 \%$ ee $166 h, R^{1}=H ; R^{2}=4-B r C_{6} H_{4} ; R^{3}=B n ; 99 \%,>20: 1 d r, 83 \%$ ee 166i, $R^{1}=H ; R^{2}=4-M_{e O C} H_{4} ; R^{3}=B n ; 99 \%,>20: 1 d r, 94 \%$ ee 166j, $R^{1}=H ; R^{2}=1$-naphthyl; $R^{3}=B n ; 98 \%$, >20:1 dr, 93\%ee 166k, $R^{1}=H ; R^{2}=3$-indolyl; $R^{3}=B n ; 98 \%, 8: 1 d r, 94 \%$ ee 166I, $R^{1}=H ; R^{2}=3$-pyridyl; $R^{3}=B n ; 99 \%, 16: 1 d r, 88 \%$ ee

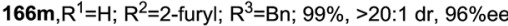
$166 n, R^{1}=H ; R^{2}=2$-thienyl; $R^{3}=B n ; 99 \%,>20: 1 \mathrm{dr}, 94 \%$ ee 166o,

$$
96 \% ; 8: 1 \mathrm{dr}, 97 \% \mathrm{ee}
$$

$166 p, R^{1}=H ; R^{2}=P h ; R^{3}=M e ; 99 \%,>20: 1 d r, 94 \% e$ 166q, $R^{1}=H ; R^{2}=P h ; R^{3}=' P r ; 99 \%, 16: 1 \mathrm{dr}, 94 \%$ ee $166 r, R^{1}=H ; R^{2}=P h ; R^{3}={ }^{n} B u ; 99 \%,>20: 1 d r, 97 \%$ ee. 166s, $\mathrm{R}^{1}=\mathrm{H} ; \mathrm{R}^{2}=\mathrm{Ph} ; \mathrm{R}^{3}=\mathrm{CH}_{2} \mathrm{CH}_{2} \mathrm{CO}_{2} \mathrm{Me} ; \mathbf{9 8 \%},>20: 1 \mathrm{dr}, 96 \%$ ee 166t, $R^{1}=H ; R^{2}=P h ; R^{3}=$ cyclohexyl; $96 \%, 17: 1 \mathrm{dr}, 97 \%$ ee 166u, $R^{1}=H ; R^{2}=P h ; R^{3}=$ allyl; 99\%, >20:1 dr, 94\%ee $166 v, R^{1}=H ; R^{2}=\mathrm{Ph} ; \mathrm{R}^{3}=4-\mathrm{MeOC}_{6} \mathrm{H}_{4} ; 90 \%, 5: 1 \mathrm{dr}, 85 \%$ ee $166 w, R^{1}=H ; R^{2}=4-B r C_{6} H_{4} ; R^{3}=4-M e O C_{6} H_{4} ; 78 \%, 5: 1 \mathrm{dr}, 90 \%$ ee $166 x, R^{1}=H ; R^{2}=P h ; R^{3}=H ; 83 \%, 7: 1 d r, 95 \%$ ee 166y, $\mathrm{R}^{1}=\mathrm{H} ; \mathrm{R}^{2}=4-\mathrm{MeOC}_{6} \mathrm{H}_{4} ; \mathrm{R}^{3}=\mathrm{H} ; 88 \%$, 9:1 dr, 98\%ee $166 z, R^{1}=H ; R^{2}=4-B r C_{6} H_{4} ; R^{3}=H ; 82 \%, 9: 1 d r, 99 \%$ ee 166aa, $R^{1}=H ; R^{2}=2$-furyl; $R^{3}=H ; 87 \%, 5: 1 d r,>99 \%$ ee 166ab, $R^{1}=4-M e O ; R^{2}=P h ; R^{3}=H ; 85 \%, 3: 1 d r,>90 \%$ ee $166 a c$,

$$
90 \%, 5: 1 \mathrm{dr},>99 \% \mathrm{ee}
$$

Scheme 51 Synthesis of spiro-1-pyrrolines 166 catalyzed by chiral silver complexes.
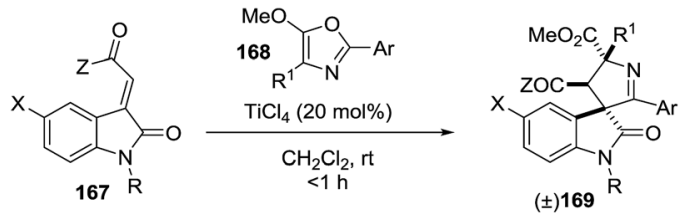

Scheme 52 Titanium(Iv)-catalyzed stereoselective synthesis of spirooxindole-1-pyrrolines.

ranging from moderate to excellent (60-95\%). The trans isomers 159 were obtained with diastereoselectivities (d.r. = trans/cis ratio) ranging from $83: 17$ d.r. $-92: 8$ d.r. and enantioselectivities from $81 \%$ ee to $98 \%$ ee. This stereodivergent synthesis offers the possibility to obtain the cis and trans pyrrolines 158 and 159 as single enantiomers.

Zang and coworkers ${ }^{108}$ used a novel three-component annulation reaction between [60]fullerene, sulfonylhydrazones 160 and nitriles 161 mediated by $\mathrm{Ag}(\mathrm{I})$ as a general entry to disubstituted [60]fullerene-fused pyrrolines 162 (Scheme 50). The reaction exhibits a broad substrate scope and excellent functional-group tolerance. As such it represents a general synthesis of fullerene-bound macromolecules.

$\mathrm{He}$, Shao and coworkers have recently developed the first enantioselective formal $[3+2]$ cycloaddition between isocyanoacetates 164 and aurone anologues 163 catalyzed by chiral silver complexes (Scheme 51). ${ }^{109}$ Several chiral precatalysts were tested in combination with $\mathrm{Ag}_{2} \mathrm{O}$ under different reaction conditions to define the optimal procedure which required the use of silver oxide (10 mol\%) and precatalyst $165(20 \mathrm{~mol} \%)$ in THF at $0{ }^{\circ} \mathrm{C}$. The protocol is robust and simple, tolerating air and moisture. This efficient and atom-economical synthetic methodology provided a set of spiro-1-pyrrolines 166 bearing three stereocenters, with excellent yields (72-99\%), and high diastero- and enantioselectivities (up to $>20: 1$ and $>99 \%$ ee, respectively). Interestingly, this protocol was successfully escalated up to a gram-reaction without a significant loss in yield or stereoselectivity. The mechanism is still under investigation.

Mukhopadhyay et al. ${ }^{110}$ reported the silver-promoted regioselective synthesis of spiro-1-pyrrolines and spiro-2-pyrrolines (see Subsection 2.2.12.: Synthesis of 2-pyrrolines by silver catalysis, Scheme 113).

2.1.9. Synthesis of 1-pyrrolines by titanium catalysis. Franz et al. ${ }^{111}$ devised access to spirocyclic oxindole 1-pyrrolines 169 in excellent yield and diastereoselectivity using a $\mathrm{TiCl}_{4}$-catalyzed formal [3+2] cycloaddition between alkylidene oxindoles 167 and 5-alkoxy-2-aryloxazoles 168 (Scheme 52). A chelating group on the nitrogen of the indole is essential for selectivity $(\mathrm{R}=\mathrm{Ac}$, $\mathrm{Cbz}$ ). Substitution at the 4-position of the oxazole afforded a spiro-1-pyrroline $\left(\mathrm{X}=\mathrm{F} ; \mathrm{Z}=\mathrm{OEt} ; \mathrm{R}^{1}=\mathrm{Me} ; \mathrm{R}=\mathrm{Ac} ; \mathrm{Ar}=\mathrm{PMP}\right)$, containing two stereogenic quaternary centers, with high diastereoselectivity $(\mathrm{dr}=94: 6)$

They extended this methodology to the synthesis of the highly functionalized pyrrolines $\mathbf{1 7 1}$ and $\mathbf{1 7 3}$ from ethyl benzylidene malonate (170) and coumarin (172) derivatives, respectively (Scheme 53 ).

The use of a chiral scandium(III)-indapybox/BArF complex provided the enantioenriched spirooxindole-1-pyrroline 174a 

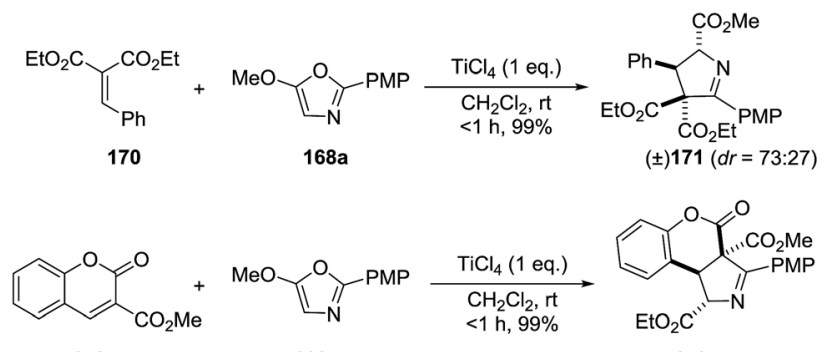

172

$( \pm) 173(d r=80: 20)$

Scheme 53 Cyclization with malonate alkylidene and coumarin electrophiles.
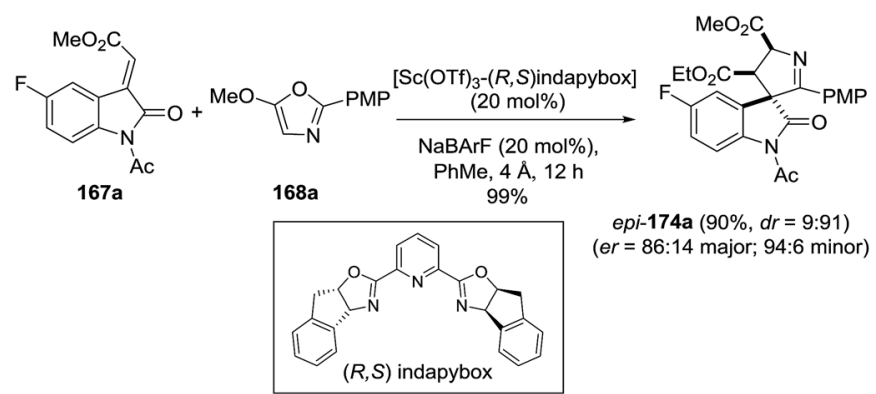

epi-174a $(90 \%, d r=9: 91)$ (er $=86: 14$ major; $94: 6$ minor)

Scheme 54 Synthesis of enantioenriched pyrroline epi-174a.

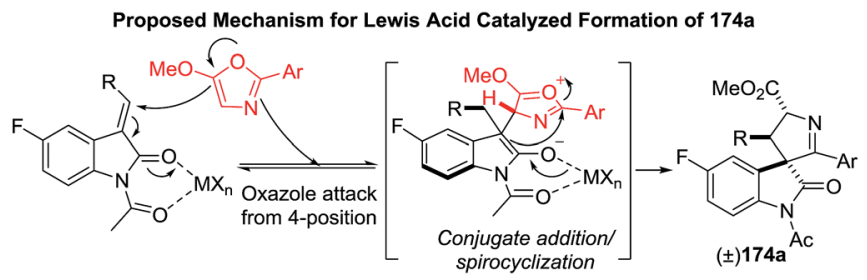

Scheme 55 Proposed mechanism for Lewis acid catalyzed spirooxindole-1-pyrrolines.

( $86: 14 \mathrm{er}$ ), and a ligand-induced reversal of diastereoselectivity was observed (Scheme 54).

The proposed mechanism is depicted in the Scheme 55.

Chiba et al. ${ }^{112}$ carried out a $\mathrm{TiCl}_{4}$-catalyzed addition of vinyl azides (176) onto $\alpha, \beta$-unsaturated carbonyl compounds to construct the 1-pyrroline (Scheme 56). Employing 3-alkylidene2-oxoindoline (175) as the $\alpha, \beta$ unsaturated carbonyl and $\mathrm{TiCl}_{4}$ (10 mol\%), the 1-pyrroline-containing spiro structures 177 were obtained in excellent yields $(72-95 \%)$. When a 2 -alkylidenemalonate (178) was used, a series of 1-pyrrolines with various aryl, alkenyl and alkyl motifs (179) were obtained in satisfactory yields $(58-75 \%)$. The proposed mechanism involves titaniumcatalyzed conjugate addition of vinyl azides to the $\alpha, \beta$ unsaturated carbonyl and subsequent denitrogenative ring-expansion of the transient azidocyclobutane intermediates formed to obtain the 1-pyrroline ring.

\subsection{Synthesis of 2-pyrrolines}

The 2-pyrrolines ring is found in many biologically-active compounds (Fig. 4). Notable examples include the cytotoxin

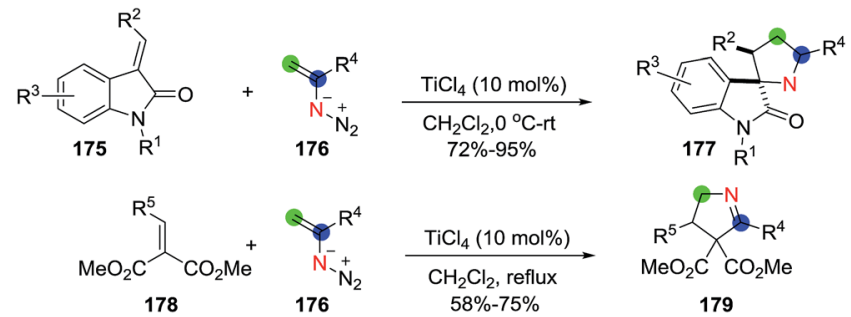

Scheme 56 Titanium-catalyzed construction of 1-pyrrolines using vinyl azides.

spirotryprostatin $\mathrm{B}(\mathbf{1 8 0}$, showing complete inhibition of the cell cycle progression of tsFT210 cells in the G2/M phase and growth inhibition toward leukemia K562 and HL-60 cells); ${ }^{7}$ the antibiotic and cytotoxin anthramycin (181, produced by Streptomyces refuineus $) ; ~$ and the synthetic, highly substituted racemic 2pyrroline 182 demonstrated to have antiproliferative activity. ${ }^{16}$

An example of 2-pyrroline used as an intermediate towards a more complex heterocycle was described by Knolker et al. ${ }^{30}$ The antibiotics pentabromopseudilin $187 \mathbf{a}$ and pentachloropseudilin $\mathbf{1 8 7 b}$ were synthesized in seven steps from the benzaldehydes 183a and 183b respectively (Scheme 57). Both syntheses made use of a silver(I)-catalyzed cyclization of $\mathrm{N}$ tosylhomopropargylamines 184a and 184b that led to 2-pyrrolines 185a and 185b. These pyrrolines were oxidized to the pyrroles 186a and 186b which were finally transformed in pentabromo- (187a) and pentachloropseudilin (187b) in $23 \%$ and $19 \%$ overall yield, respectively.

2.2.1. Synthesis of 2-pyrrolines by copper catalysis. Zhu et al. described the copper-catalyzed oxidative cyclization of

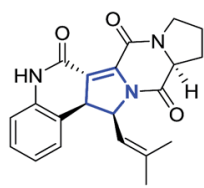

180: Cytotoxin

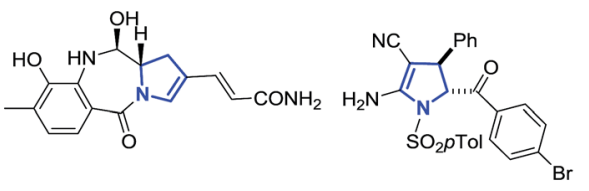

181: Antiproliferative

182: Antiproliferative
Fig. 4 Selected examples of biologically active 2-pyrrolines.

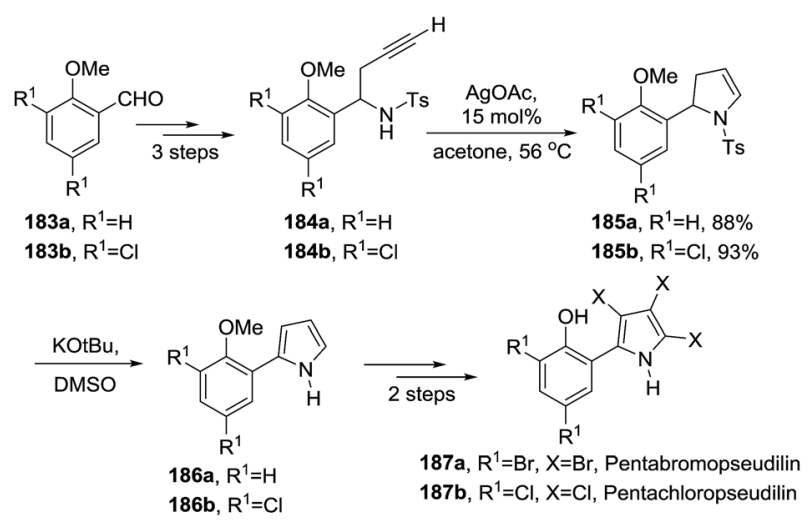

Scheme 57 Synthesis of pentabromopseudilin 187a and pentachloropseudilin 187b through the 2-pyrrolines $186 \mathrm{a}$ and $186 \mathrm{~b}$, respectively. 


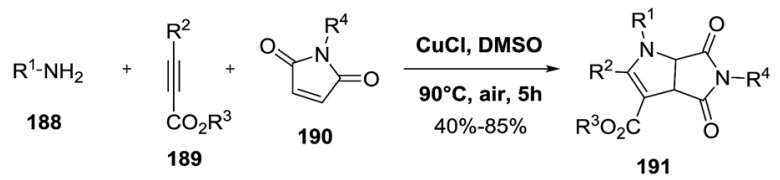

Scheme 58 Cu-catalyzed synthesis of fully substituted 2-pyrrolines.

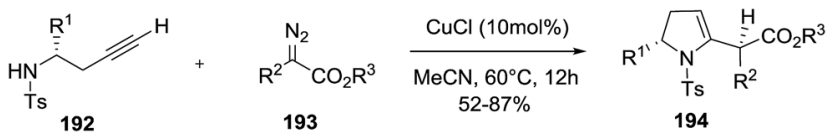

Scheme 59 Copper-catalyzed tandem annulation towards 2pyrrolines.

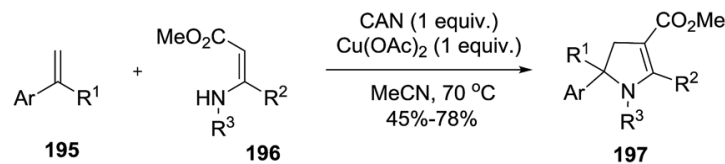

Scheme 60 Oxidative radical cyclization of $N$-sulfonyl $\beta$-enamino esters with alkenes towards 2-pyrrolines.

maleimides 190 with different amines 188 and alkynyl esters 189 to give a variety of 2-pyrrolines 191 (Scheme 58). ${ }^{113} \mathrm{CuCl}$ in dimethylsulfoxide (DMSO) at $90{ }^{\circ} \mathrm{C}$ in presence of air were the most effective conditions. The presence of oxygen in the reaction medium was necessary to effect the transformation. The reaction has a good tolerance towards different functional groups, allowing both N-protected and free $\mathrm{NH}$ maleimide, amines containing electron-donating and electron-withdrawing substituents, and alkyl- as well as aryl-substituted alkynyl esters. In all cases, the desired product was obtained in good to moderate yield (40-85\%). A radical mechanism was proposed.

Sun et al. reported the copper-catalyzed 5-exo-dig hydroamination of homopropargyl sulfonamides 192 with diazo compounds 193 to give 2-pyrrolines 194 (Scheme 59). ${ }^{114}$ The possible first step involves formation of allenoate intermediates by metal-catalyzed cross-coupling of an alkyne with diazo compounds which then undergo a 5-exo-dig intramolecular hydroamination.

When chiral homopropargyl sulfonamides with a variety of substituent adjacent to the $\mathrm{N}$-atom are subjected to the optimal reaction conditions $(10 \mathrm{~mol} \% \mathrm{CuCl}$ in acetonitrile), 2,5 disubstituted 2-pyrrolines are obtained with good yields (52-87\%) and good diasteroselectivity (dr typically $4: 1$ ). A library of twenty-four 2,5-disubstituted 2-pyrrolines 194 was synthesized.

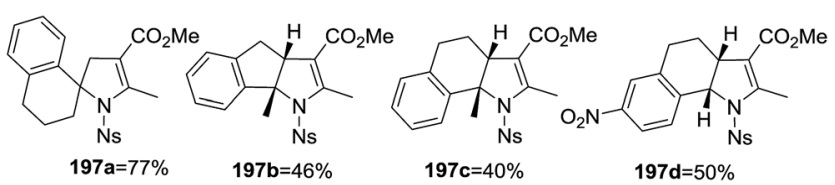

Scheme 61 Spirocyclic and tricyclic dihydropyrrole obtained by the reaction with cyclic alkenes.

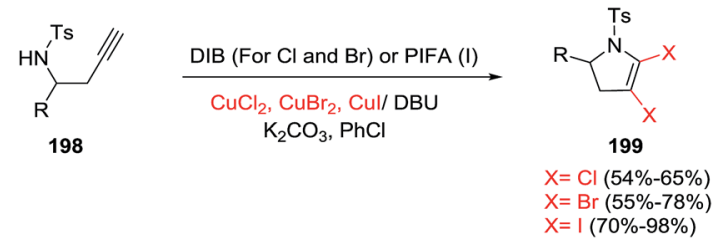

Scheme 62 Transformation of homopropargyl sulfonamides into dihalo-2-pyrrolines.

An alternative synthesis of multi-substituted 2-pyrrolines 197 was described by Yoshida et al. in 2016 (Scheme 60). ${ }^{115}$ They reported the oxidative radical cyclization of $N$-sulfonyl $\beta$ enamino esters 196 with different aryl-substituted alkenes 195. When cyclic alkenes are used, spirocyclic and tricyclic dihydropyrroles like 197a, 197b, 197c, and 197d were obtained (Scheme 61). Under optimal conditions with ceric ammonium nitrate and $\mathrm{Cu}(\mathrm{OAc})_{2}$ as oxidants, the desired products were obtained in moderate to high yields (45-78\%).

In 2017, the Xiang research group reported the synthesis of 4,5-dihalo-substituted 2,3-dihydropyrroles 199 from a variety of homopropargyl sulfonamides 198 (Scheme 62). ${ }^{116}$ This transformation uses two equivalents of copper halides and one equivalent of a hypervalent iodine compound, in the presence of an organic base (such as DBU) and an inorganic base (such as $\mathrm{K}_{2} \mathrm{CO}_{3}$ ) in chlorobenzene as the solvent. The combination of CuI and PIFA gives the 2,3-dihydro-4,5-diiodopyrroline products. The dibromopyrrolines (dichloropyrrolines) are obtained using $\mathrm{CuBr}_{2}\left(\mathrm{CuCl}_{2}\right)$ and oxidant DIB $\left[\mathrm{PhI}(\mathrm{OAc})_{2}\right]$. A breadth of different aromatic, heteroaromatic, and alkyl-substituted alkynyl amines under the former conditions give the diiodopyrrolines in very good to excellent yields, defining a broad scope to the reaction. In general, the diiodopyrrolines were obtained in higher yields than the dibromopyrroline, with the dichloropyrroline obtained in the lowest yield.

Wang et al. reported a copper-catalyzed synthesis of 2-fulleropyrrolines 201 bearing a trisubstituted or a tetrasubstituted $\mathrm{C}=\mathrm{C}$ bond promoted by copper from $\alpha$-amino ketones 200 and

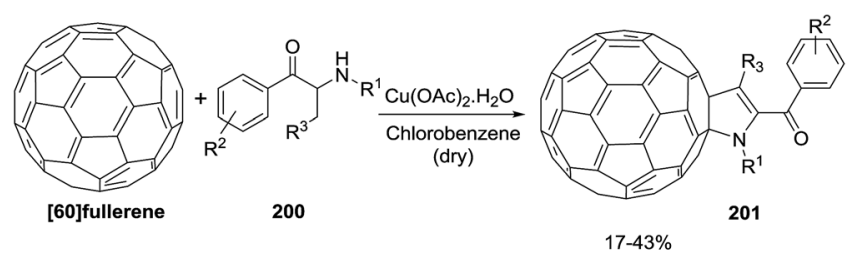

201a, $R^{1}=P h ; R^{2}=H ; R^{3}=H ; 5 h ; 34 \%$ $201 b, R^{1}=2-M_{e C} H_{4} ; R^{2}=H ; R^{3}=H ; 5 h ; 34 \%$ 201c, $\mathrm{R}^{1}=3-\mathrm{MeC}_{6} \mathrm{H}_{4} ; \mathrm{R}^{2}=\mathrm{H} ; \mathrm{R}^{3}=\mathrm{H} ; 5 \mathrm{~h} ; 33$ 201d, $R^{1}=4-M_{e C} \mathrm{H}_{4} ; \mathrm{R}^{2}=\mathrm{H} ; \mathrm{R}^{3}=\mathrm{H} ; 5 \mathrm{~h} ; 31 \%$ 201e, $R^{1}=4-M_{e O C} \mathrm{H}_{4} ; \mathrm{R}^{2}=\mathrm{H} ; \mathrm{R}^{3}=\mathrm{H} ; 3 \mathrm{~h} ; 34 \%$ 201f, $\mathrm{R}^{1}=4-\mathrm{ClC}_{6} \mathrm{H}_{4} ; \mathrm{R}^{2}=\mathrm{H} ; \mathrm{R}^{3}=\mathrm{H} ; 5 \mathrm{~h} ; 31 \%$ $201 \mathrm{~g}, \mathrm{R}^{1}=3,5-d i-\mathrm{MeC}_{6} \mathrm{H}_{3} ; \mathrm{R}^{2}=\mathrm{H} ; \mathrm{R}^{3}=\mathrm{H} ; 3 \mathrm{~h} ; 40 \%$ $201 \mathrm{~h}, \mathrm{R}^{1}=3,5-d i-\mathrm{MeC}_{6} \mathrm{H}_{3} ; \mathrm{R}^{2}=4-\mathrm{Me} ; \mathrm{R}^{3}=\mathrm{H} ; 3 \mathrm{~h} ; 37 \%$ 201i, $R^{1}=$ PPr; $R^{2}=H ; R^{3}=H ; 3 h ; 17 \%$ $201 \mathrm{j}, \mathrm{R}^{1}=\mathrm{Ph} ; \mathrm{R}^{2}=\mathrm{H} ; \mathrm{R}^{3}=\mathrm{Me} ; 3 \mathrm{~h} ; 18 \%$ $201 \mathrm{k}, \mathrm{R}^{1}=3,5-d i-\mathrm{MeC}_{6} \mathrm{H}_{3} ; \mathrm{R}^{2}=\mathrm{H} ; \mathrm{R}^{3}=\mathrm{Me} ; 3 \mathrm{~h} ; 22 \%$ 201I, $R^{1}=\mathrm{Ph} ; \mathrm{R}^{2}=\mathrm{H} ; \mathrm{R}^{3}=\mathrm{Ph} ; 3 \mathrm{~h} ; 40 \%$ $201 \mathrm{~m}, \mathrm{R}^{1}=3,5-d i-\mathrm{MeC}_{6} \mathrm{H}_{3} ; \mathrm{R}^{2}=\mathrm{H} ; \mathrm{R}^{3}=\mathrm{Ph} ; 3 \mathrm{~h} ; 43 \%$

Scheme 63 Synthesis of 2-fulleropyrrolines 201. 
[60]fullerene (Scheme 63) ${ }^{117}$ This is the first synthesis of a 2fulleropyrrolines with $\mathrm{R}^{3}=\mathrm{H}$. The yields are in the range of $17 \%$ to $43 \%$.

Recently, Zhang et al. disclosed an enantioselective [3 + 2] cycloaddition synthesis of 2-pyrrolines 204 bearing a trifluoromethylated all-carbon quaternary stereocenter from $\alpha$ tert-butyl isocyanoacetates 203 and $\beta$-trifluoromethyl $\beta, \beta$ disubstituted enones 202 in the presence of a copper catalysis (Scheme 64). ${ }^{118}$ The combination of $\mathrm{Cu}\left(\mathrm{CH}_{3} \mathrm{CN}\right)_{4} \mathrm{BF}_{4}$ and the chiral ligand (R)-DTBM-SEGPHOS provided high diastereoselectivities (>20:1) and enantioselectivities (50-96\% ee) along with very good yields (57-99\%). The protocol tolerates a variety of enones 202 bearing electron-rich or -poor substituted aryl groups $\left(\mathrm{R}^{1}\right)$ but good diasteroselectivities are only obtained when $\mathrm{R}=\mathrm{Me}$. The presence of the $\beta$-trifluoromethyl group in the enone $\mathbf{2 0 2}$ is necessary for attaining high reactivity and stereocontrol.

Fan et al. reported a copper-catalyzed cascade reaction synthesis of 3-acyl-2-pyrrolines 207 from pyrrolidines 205 and 2oxo-2-arylacetic acids 206 (Scheme 65). ${ }^{119}$ The reaction is believed to occur through a $\mathrm{C}\left(\mathrm{sp}^{3}\right)-\mathrm{H}$ bond dehydrogenation of the pyrrolidine to give an enamine intermediate which is subsequently cross-coupled with the acyl species coming from the decarboxylation of 2-oxo-2-arylacetic acid 206. It is postulated that the copper catalyst plays an essential role in the dehydrogenation, decarboxylation and cross coupling steps. $\mathrm{N}$ Aryl substituted pyrrolidines 205 and various 2-oxo-2-arylacetic acids 206 deliver the 3-acyl-2-pyrrolines 207 in good yields (58-68\%). $N$-Alkyl substituted pyrrolidines give pyrroles instead of 3-acyl-2-pyrrolines.

2.2.2. Synthesis of 2-pyrrolines by gold catalysis. The Rutjes group developed an enantioselective gold-catalyzed 5-endo-dig cyclization into the 2,3-dihydro-3-hydroxy-substituted 2-pyrrolines 209 using the alkynyl-substituted amino alcohols 208 as

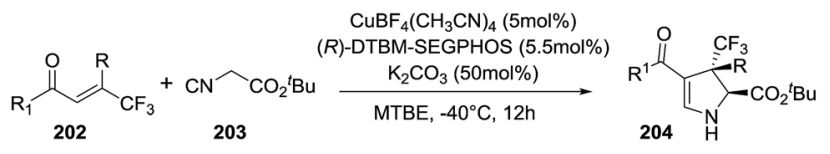

204a, R=Me; $\mathrm{R}^{1}=4-\mathrm{ClC}_{6} \mathrm{H}_{4} ;>20: 1 ; e e=93 \% ; 94 \%$ 204b, $\mathrm{R}=\mathrm{Me} ; \mathrm{R}^{1}=4-\mathrm{BrC}_{6} \mathrm{H}_{4} ;>20: 1 ; e e=94 \% ; 80 \%$ 204c, $\mathrm{R}=\mathrm{Me} ; \mathrm{R}^{1}=4-\mathrm{FC}_{6} \mathrm{H}_{4} ;>20: 1$; ee=90\%; $85 \%$ 204d, $\mathrm{R}=\mathrm{Me} ; \mathrm{R}^{1}=4-\mathrm{NO}_{2} \mathrm{C}_{6} \mathrm{H}_{4} ;>20: 1 ;$ ee= $90 \% ; 99 \%$ 204e, $\mathrm{R}=\mathrm{Me} ; \mathrm{R}^{1}=4-\mathrm{CNC}_{6} \mathrm{H}_{4} ;>20: 1 ; \boldsymbol{e}=\mathbf{9}=94 \% ; 91 \%$ 204f, $\mathrm{R}=\mathrm{Me} ; \mathrm{R}^{1}=4-\mathrm{CF}_{3} \mathrm{C}_{6} \mathrm{H}_{4} ;>20: 1 ;$ ee $=94 \% ; 89 \%$ 204g, $\mathrm{R}=\mathrm{Me} ; \mathrm{R}^{1}=4-\mathrm{MeO}_{2} \mathrm{CC}_{6} \mathrm{H}_{4} ;>20: 1 ; e e=93 \% ; 85 \%$ 204h, $\mathrm{R}=\mathrm{Me} ; \mathrm{R}^{1}=4-\mathrm{CH}_{3} \mathrm{C}_{6} \mathrm{H}_{4} ;>20: 1 ;$ ee $=91 \% ; 79 \%$ 204i, $\mathrm{R}=\mathrm{Me} ; \mathrm{R}^{1}=4-\mathrm{MeOC}_{6} \mathrm{H}_{4} ;>20: 1 ;$ ee=93\%; $68 \%$ 204j, $\mathrm{R}=\mathrm{Me} ; \mathrm{R}^{1}=4-\mathrm{PhC}_{6} \mathrm{H}_{4} ;>20: 1 ;$ ee $=96 \% ; 87 \%$ 204k, R=Me; $\mathrm{R}^{1}=\mathrm{C}_{6} \mathrm{H}_{4} ;>20: 1 ;$ ee $=90 \% ; 70 \%$ 204I, R=Me; $\mathrm{R}^{1}=3,4-\mathrm{Cl}_{2} \mathrm{C}_{6} \mathrm{H}_{4} ;>20: 1$; ee=93\%; $85 \%$ 204m, R=Me; $\mathrm{R}^{1}=2$-naphthyl; >20:1; ee=90\%; 71\% 204n, R=Me; $R^{1}=2$-furyl; >20:1; ee $=90 \% ; 76 \%$ 204o, R=Et; $\mathrm{R}^{1}=4-\mathrm{ClC}_{6} \mathrm{H}_{4} ; 6: 1 ; e e=90 \% ; 98 \%$ 204p, $\mathrm{R}=\mathrm{Bu} ; \mathrm{R}^{1}=4-\mathrm{ClC}_{6} \mathrm{H}_{4} ; 6: 1 ; e e=90 \% ; 76 \%$ 204q. $\mathrm{R}=\mathrm{Ph} ; \mathrm{R}^{1}=4-\mathrm{ClC}_{6} \mathrm{H}_{4} ; 7: 1 ;$ ee $=94 \% ; 76 \%$ 204r, R=4- $\mathrm{ClC}_{6} \mathrm{H}_{4} ; \mathrm{R}^{1}=4-\mathrm{ClC}_{6} \mathrm{H}_{4} ; 6: 1 ;$ ee=93\%; $74 \%$ 204s, $\mathrm{R}=4-\mathrm{FC}_{6} \mathrm{H}_{4} ; \mathrm{R}^{1}=4-\mathrm{ClC}_{6} \mathrm{H}_{4} ; 7: 1 ; e=95 \% ; 75 \%$ 204t, $\mathrm{R}=4-\mathrm{CF}_{3} \mathrm{C}_{6} \mathrm{H}_{4} ; \mathrm{R}^{1}=4-\mathrm{ClC}_{6} \mathrm{H}_{4} ; 7: 1 ; e e=95 \% ; 70 \%$ 204u, R=4- $\mathrm{MeOC}_{6} \mathrm{H}_{4} ; \mathrm{R}^{1}=4-\mathrm{ClC}_{6} \mathrm{H}_{4} ;>20: 1 ; e e=82 \% ; 57 \%$ 204v, R=Me; $\mathbf{R}^{1}=$ cyclohexyl; $6: 1 ;$ ee=61\%; $77 \%$ 204w, R=Me; $\mathrm{R}^{1}=\mathrm{PhCH}_{2} \mathrm{CH}_{2} ; 5: 1 ; e e=50 \% ; 67 \%$

Scheme 64 Synthesis of 2 -pyrrolines 204 by enantioselective [3 +2 ] cycloaddition.

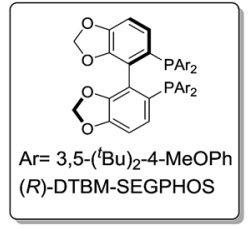

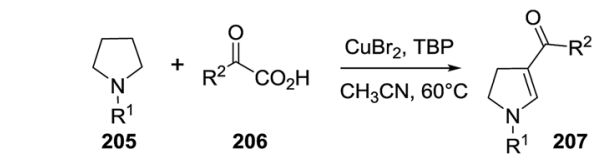

207a, $\mathrm{R}^{1}=\mathrm{Ph} ; \mathrm{R}^{2}=\mathrm{Ph} ; 68 \%$ 207b, $\mathrm{R}^{1}=4-\mathrm{ClC}_{6} \mathrm{H}_{4} ; \mathrm{R}^{2}=\mathrm{Ph} ; 67 \%$

207e, $\mathrm{R}^{1}=\mathrm{Ph} ; \mathrm{R}^{2}=3-\mathrm{BrC}_{6} \mathrm{H}_{4} ; 64 \%$ 207f, $R^{1}=\mathrm{Ph} ; \mathrm{R}^{2}=4-\mathrm{MeC}_{6} \mathrm{H}_{4} ; 62 \%$ 207c, $\mathrm{R}^{1}=3,5-d i-\mathrm{MeC}_{6} \mathrm{H}_{3} ; \mathrm{R}^{2}=\mathrm{Ph} ; 61 \% 207 \mathrm{~g}, \mathrm{R}^{1}=\mathrm{Ph} ; \mathrm{R}^{2}=4-\mathrm{ClC}_{6} \mathrm{H}_{4} ; 58 \%$ 207d, $R^{1}=P h ; R^{2}=3-M_{e} C_{6} H_{4} ; 58 \% \quad 207 h, R^{1}=P h ; R^{2}=4-B^{2} C_{6} H_{4} ; 60 \%$.

Scheme 65 Copper-catalyzed synthesis of 3-acyl-2-pyrrolines 207.

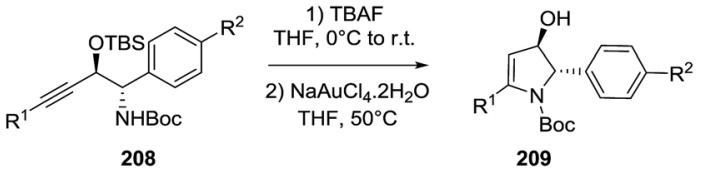

209a, $\mathrm{R}^{1}=\mathrm{Ph} ; \mathrm{R}^{2}=\mathrm{H} ; R, S(87 \%)$ 209b, $\mathrm{R}^{1}=\mathrm{Ph} ; \mathrm{R}^{2}=\mathrm{F} ; \mathrm{R}, \mathrm{S}(8 \mathrm{O} \%)$

209e, $\mathrm{R}^{1}=3-\mathrm{MeOC}_{6} \mathrm{H}_{4} ; \mathrm{R}^{2}=\mathrm{F} ; \mathrm{S}, \mathrm{R}(82 \%)$ 209f, $\mathrm{R}^{1}=3-\mathrm{MeOC}_{6} \mathrm{H}_{4} ; \mathrm{R}^{2}=\mathrm{Cl} ; \mathrm{S}, R(79 \%)$

209c, $R^{1}=4-M_{e O C} \mathrm{H}_{4} ; R^{2}=F ; S, R(91 \%) \quad 209 g, R^{1}=4-F_{6} H_{4} ; R^{2}=C l ; S, R(76 \%)$

209d, $\mathrm{R}^{1}=3-\mathrm{MeOC}_{6} \mathrm{H}_{4} ; \mathrm{R}^{2}=\mathrm{H} ; \mathrm{S}, \mathrm{R}(81 \%) \quad 209 \mathrm{~h}, \mathrm{R}^{1}=3$-piperonyl; $\mathrm{R}^{2}=\mathrm{H} ; \mathrm{S}, \mathrm{R}(83 \%)$

Scheme 66 Gold-catalyzed cyclization of substituted alkyne-containing amino alcohols towards 2-pyrrolines.

the starting material (Scheme 66). ${ }^{120}$ This starting material originated through enzyme-catalyzed hydrocyanation of the $\alpha, \beta$ acetylenic aldehydes. Deprotection of the hydroxyl group with TBAF followed by reaction with $\mathrm{NaAuCl}_{4} \cdot 2 \mathrm{H}_{2} \mathrm{O}(10 \mathrm{~mol} \%)$ in THF at $50{ }^{\circ} \mathrm{C}$ gave the 4-hydroxy-2-pyrrolines 209 in good yields over the two steps.

Jørgensen reported ${ }^{121}$ an enantioselective synthesis of 2pyrrolines 212 from propargyl malononitrile (211) and $N$-Bocprotected imines $\mathbf{2 1 0}$ through an organocatalytic Manich-type reaction followed by gold-catalyzed alkyne hydroamination and subsequent isomerization (Scheme 67). This one-pot sequential protocol that combine transition-metal catalysis with organocatalysis furnished the 2-pyrrolines 212 in good yields (up to $80 \%$ ), high selectivities endo/exo $(10>1)$, and enantioselectivities up to $88 \%$. The protocol works only for terminal alkynes. Once the organocatalyst $\mathbf{2 1 3 b}$ forms the Mannich product, the latter is protonated by the excess of $p$ $\mathrm{TsOH}$, and in this way deactivation of the gold catalyst is prevented. The two catalytic systems are compatible with one-pot operation.

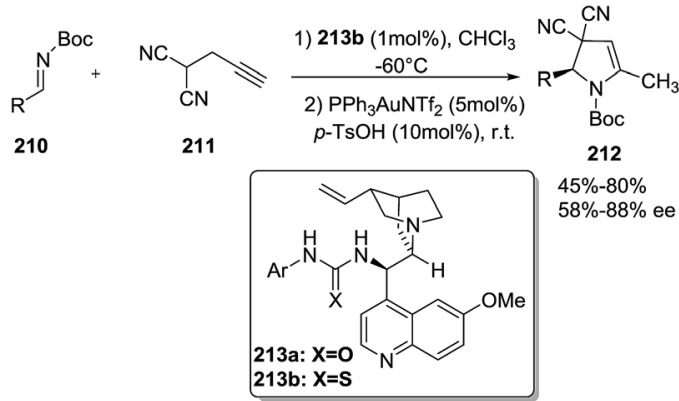

Scheme 67 Asymmetric one-pot sequential organo- and gold catalysis for the enantioselective synthesis of 2-pyrrolines 212. 


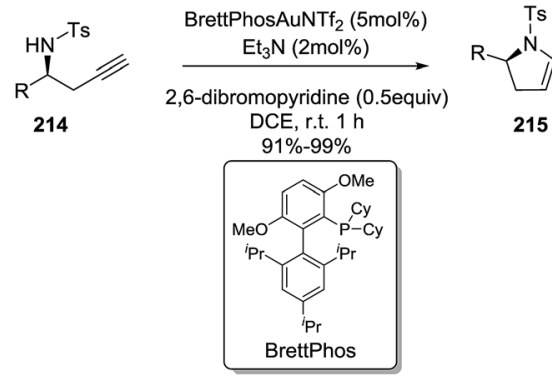

Scheme 68 Enantioselective synthesis of 2-pyrrolines via gold-catalyzed cycloisomerization of chiral homopropargyl sulfonamides.

Ye et al. reported an enantioselective gold-catalyzed synthesis of 2-pyrrolines $\mathbf{2 1 5}$ from chiral homopropargyl sulfonamides 214 using a combination of bases as catalytic additives. ${ }^{\mathbf{1 2 2}}$ The intramolecular hydroamination occurs via a 5endo-dig cyclization, through an anti-Markovnikov addition. The optimal reaction conditions use BrettPhos $\cdot \mathrm{AuNTf}_{2}$ ( $5 \mathrm{~mol} \%$ ), a combination of $\mathrm{Et}_{3} \mathrm{~N}(2 \mathrm{~mol} \%)$ and 2,6-dibromopyridine ( 0.5 equivalent) as the basic additives, in 1,2-dichloroethane at room temperature (Scheme 68). The 2,3dihydropyrroles 215 are isolated in excellent yields and without epimerization of the stereocenter. This practical procedure offers a route to both 2-pyrrolines enantiomers, simply by selecting the starting chiral homopropargyl sulphonamides $(R$ or $S)$ 214. The reaction showed wide generality. Diversely substituted homopropargyl sulfonamides produced the 2-pyrrolines in excellent yields (91-99\%).

Later, the same group reported an enantioenriched access to multisubstituted 2-pyrrolines 218 by a combination of gold catalysis and visible-light photoredox catalysis. ${ }^{\mathbf{1 2 3}}$ Using different chiral homopropargyl sulfonamides 216 and a variety of aryldiazonium salts 217 in the presence of $\mathrm{Ph}_{3} \mathrm{P} \cdot \mathrm{AuCl}$ and a visible light photocatalysts such $\mathrm{Ru}(\mathrm{bpy})_{3}(\mathrm{PF} 6)_{2}$, and irradiating with $13 \mathrm{~W}$ white LEDs, a bis-arylative 5-endo-dig cyclization led to enantioenriched 2,3-dihydropyrroles with high enantiomeric excesses (96-99\% ee) and moderate yields (54-89\%) (Scheme 69). The proposed mechanism is an $\mathrm{Au}(\mathrm{I}) / \mathrm{Au}(\mathrm{III})$ redox cycle accomplished by visible-light photoredox catalysis, without using a strong oxidant, and accomplished in a mild and selective manner.

Pyne et al. reported the cyclization of $\beta$-amino allenes 219 by gold and silver catalysis to afford the pirrolines 220a via a 5endo-dig cyclization to $220 \mathrm{~b}$ and subsequent isomerization to the more stable isomer 220a (Scheme 70). ${ }^{124}$ Under gold catalysis, the optimal conditions consist of using $10 \mathrm{~mol} \%$ of $\left(\mathrm{PPh}_{3}\right)$ $\mathrm{AuCl} / \mathrm{AgBF}_{4}$ in acetonitrile at room temperature. $\beta$-amino allenes 219 provided the pyrroline 220a and the pyrrolidine $220 b$ with $70 \%$ isolated yield in a ratio $(220 \mathrm{a} / 220 \mathrm{~b}=1.5: 1)$. Treating the same allene with $\mathrm{Ag}_{2} \mathrm{O}$ in acetone a room temperature generated a mixture $\mathbf{2 2 0 a} / \mathbf{2 2 0 b}=1: 1.28$ in $61 \%$ overall yield. The $\beta$-amino allene 221, substituted with the conjugated cinnamyl group, was subjected to the optimized gold catalyzed conditions obtaining a mixture between the pyrroline 222a and pirrolidine 222b in low yield but favoring the

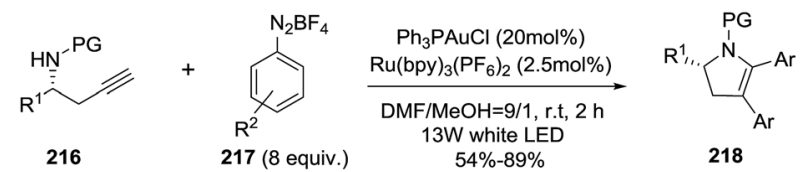

Scheme 69 Dual photoredox/gold catalysis towards enantioenriched 2-pyrrolines.

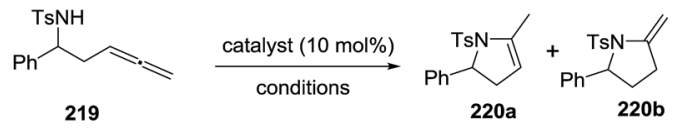

Catalyst: $\left(\mathrm{PPh}_{3}\right) \mathrm{AuCl} / \mathrm{AgBF}_{4}$, conditions: $\mathrm{CH}_{3} \mathrm{CN}, \mathrm{rt}, 30 \mathrm{~min}$, yield: $70 \%$ 220a/220b (1.5:1) Catalyst: $\mathrm{Ag}_{2} \mathrm{O}$, conditions: Acetone, rt, $28 \mathrm{~h}$, yield: $61 \% 220 \mathrm{a} / 220 \mathrm{~b}(1: 2.8)$

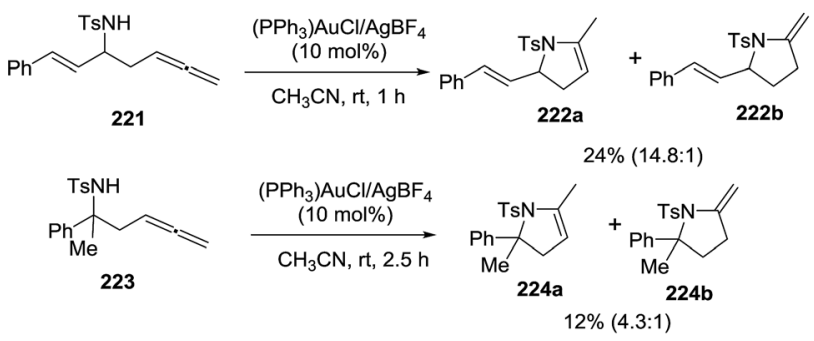

Scheme 70 Gold- and silver-catalysed cyclisation reactions of $\beta$ amino allenes.

formation of 222a. Under optimized conditions, the $\beta, \beta$-disubstituted allene $\mathbf{2 2 3}$ produced the pyrroline $\mathbf{2 2 4 a}$ and the isomer $224 b$ in low yield $(12 \%)$ in a ratio $224 a / 224 b=4.3: 1$.

Hou et al. ${ }^{125}$ have studied the gold and palladium catalyzed intramolecular hydroamination of $N$-(3-butynyl) sulfonamides leading to 2,3-dihydropyrroles (see Scheme 83, Subsection 2.2.7.: Synthesis of 2-pyrrolines by palladium catalysis).

2.2.3. Synthesis of 2-pyrrolines by iron catalysis. Tang et al. reported the diastereoselective synthesis of trans-2,3-disubstituted 2-pyrrolines 227 from $\alpha, \beta$-unsaturated imines 225 and alkyl diazoacetate 226 in presence of catalytic tetra( $p$-chlorophenyl)porphyrin iron chloride (Fe(Tcpp)Cl) and 4-picoline (Scheme 71). ${ }^{\mathbf{1 2 6}}$ The formal $[4+1]$ annulations occur via an iron carbenoid intermediate, which is readily available from alkyl diazo acetate. The yields are ranging from $60 \%$ to $92 \%$ with diastereoselectivities higher than $50: 1$.

The development of iron-catalyzed organic reactions is attractive since iron is an abundant, cheap, environmentally friendly, and efficient catalyst. In 2014, Bi et al. described the $\mathrm{FeCl}_{3}$ catalyzed stereoselective synthesis of 5-(aryl)alkylidene4,5-dihydropyrroles 230 through a $[4 \mathrm{C}+1 \mathrm{~N}]$ cyclization of 4 alkynyl ketones 228 with primary amines 229 (Scheme 72). ${ }^{127}$

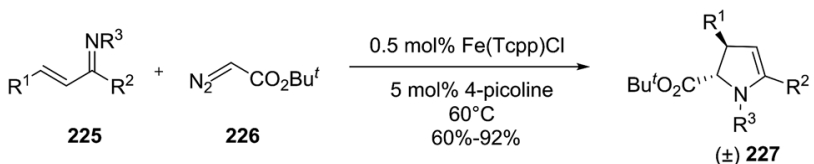

Scheme 71 Diastereoselective synthesis of 2-pyrrolines via a catalytic formal [4 + 1] annulations. 


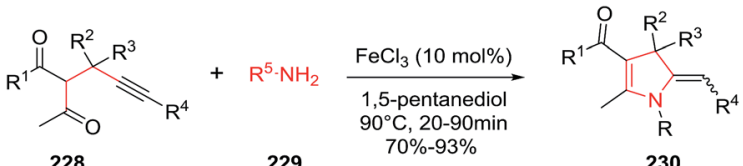

Scheme 72 Iron-catalyzed $[4 C+1 N]$ cyclization of 4-acetylenic ketones with primary amines.

The optimized conditions used $\mathrm{FeCl}_{3}(10 \mathrm{~mol} \%)$ in 1,5-pentanediol at $90{ }^{\circ} \mathrm{C}$. The protocol tolerates a wide range of 4-alkynyl ketones to give the multisubstituted 2-pyrroline 230 in very good to excellent yields (70-93\%) and high stereoselectivities $(Z /$ $E$ ratio typically $1: 0.05)$. The $Z$-isomer of the exocyclic alkene dominates the product mixture. The procedure is also useful to obtain 3-spirodihydropyrroles 230a and 230b from the cyclization of 4-alkynyl ketones containing a cyclic quaternary carbon center, again using primary amines to initiate the cyclization (Scheme 73).

Recently, the Rueping group reported an intramolecular hydroamination of $\alpha$-allenic amines 231 to 2-pyrrolines 233 catalyzed by an air- and moisture-stable iron cyclopentadienone complex (Scheme 74). ${ }^{128}$ The protocol consists in adding 5 mol\% of iron complex 232 and 6 mol\% of trimethylamine $N$ oxide to Cbz-allenic amines 231 in THF at $70{ }^{\circ} \mathrm{C}$ for $24 \mathrm{~h}$. The yields obtained varied from $57 \%$ to $86 \%$ of the 2-pyrrolines 233 .

2.2.4. Synthesis of 2-pyrrolines by iridium catalysis. Zhang et al. described an iridium-catalyzed synthesis of 2-pyrrolines 236 from vinylaziridines 234 and $\alpha$-unsubstituted 1,3-dicarbonyls 235 through a domino-ring-opening cyclization in good

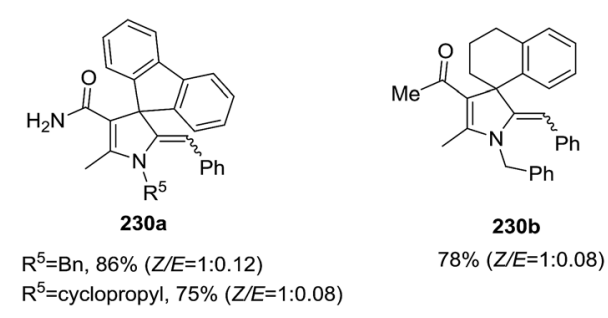

Scheme 73 Synthesis of 3-spiro 2-pyrrolines by Fe-catalyzed $[4 \mathrm{C}+$ $1 \mathrm{~N}]$ cyclization.

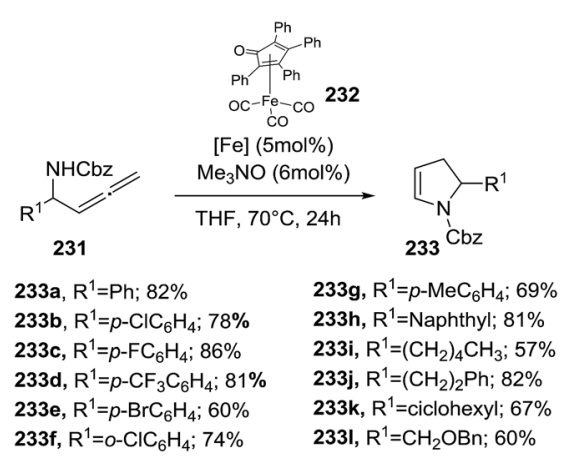

Scheme 74 Iron-catalyzed hydroamination of $\alpha$-allenic amines 231 towards 2-pyrrolines 233.

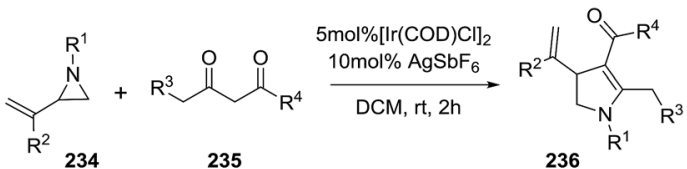

236a, $R^{1}=T s ; R^{2}=M e ; R^{3}=H ; R^{4}=M e ; 85 \%$ 236b, $R^{1}=\mathrm{Ts} ; \mathrm{R}^{2}=\mathrm{CF}_{3} ; \mathrm{R}^{3}=\mathrm{H} ; \mathrm{R}^{4}=\mathrm{Me} ; 60 \%$ 236c, $R^{1}=\mathrm{Ts} ; \mathrm{R}^{2}=n-\mathrm{Bu} ; \mathrm{R}^{3}=\mathrm{H} ; \mathrm{R}^{4}=\mathrm{Me} ; 84 \%$ 236d, $\mathrm{R}^{1}=\mathrm{Ts} ; \mathrm{R}^{2}=i-\mathrm{Pr} ; \mathrm{R}^{3}=\mathrm{H} ; \mathrm{R}^{4}=\mathrm{Me} ; 82 \%$ 236e, $R^{1}=\mathrm{Ts} ; \mathrm{R}^{2}=\mathrm{C}_{6} \mathrm{H}_{5} ; \mathrm{R}^{3}=\mathrm{H} ; \mathrm{R}^{4}=\mathrm{Me} ; 89 \%$ 236g, $R^{1}=\mathrm{Ts} ; \mathrm{R}^{2}=\mathrm{H} ; \mathrm{R}^{3}=\mathrm{H} ; \mathrm{R}^{4}=\mathrm{Me} ; 66 \%$ 236h, $R^{1}=\mathrm{Ts} ; \mathrm{R}^{2}=4-\mathrm{MeOC}_{6} \mathrm{H}_{4} ; \mathrm{R}^{3}=\mathrm{H} ; \mathrm{R}^{4}=\mathrm{Me} ; 88 \%$

236i, $R^{1}=\mathrm{Ns} ; \mathrm{R}^{2}=\mathrm{H} ; \mathrm{R}^{3}=\mathrm{H} ; \mathrm{R}^{4}=\mathrm{Me} ; 62 \%$ 236j, $R^{1}=M s ; R^{2}=H ; R^{3}=H ; R^{4}=M e ; 55 \%$ 236k, $R^{1}=T s ; R^{2}=M e ; R^{3}=M e ; R^{4}=E t ; 61 \%$ 236I, $R^{1}=T s ; R^{2}=M e ; R^{3}=H ; R^{4}=E t ; 71 \%$ 236m, $\mathrm{R}^{1}=\mathrm{Ts} ; \mathrm{R}^{2}=\mathrm{Me} ; \mathrm{R}^{3}=\mathrm{H} ; \mathrm{R}^{4}=i-\mathrm{Bu} ; 65 \%$ 236n, $R^{1}=$ Ts; $R^{2}=M e ; R^{3}=H ; R^{4}=C p r ; 49 \%$ 236o, $R^{1}=\mathrm{Ts} ; \mathrm{R}^{2}=\mathrm{Me} ; \mathrm{R}^{3}=\mathrm{H} ; \mathrm{R}^{4}=\mathrm{OEt} ; 54 \%$

Scheme 75 Reaction between vinylaziridines 234 and 1,3-dicarbonyls 235 towards 2-pyrrolines 235.

yields (49-89\%)(Scheme 75). ${ }^{129}$ The reaction conditions involve the use of $5 \mathrm{~mol} \%$ of $[\operatorname{Ir}(\mathrm{COD}) \mathrm{Cl}]_{2}, 10 \mathrm{~mol} \%$ of $\mathrm{AgSbF}_{6}$ as additive in dichloroethane at room temperature for $2 \mathrm{~h}$. Alkyl or aryl substituents on the vinylaziridine $\left(\mathrm{R}^{2}\right)$ are well tolerated. Regarding the $\mathrm{N}$-protecting group $\left(\mathrm{R}^{1}\right)$, tosyl, nosyl and mesyl provide the 2-pyrrolines with similar yields. Symmetrical and unsymmetrical 1,3 diketones produced the corresponding 2pyrrolines with excellent regioselectivity. $\beta$-ketoesters are suitable substrates.

2.2.5. Synthesis of 2-pyrrolines promoted by manganese. Zheng et al. disclosed the first oxidative cyclization promoted by $\mathrm{Mn}(\mathrm{OAc})_{3}$ to access to polysubstituted 2-pyrrolines $\mathbf{2 4 0}$ from anilines 237, alkynes esters 238 and alkenes 239 (Scheme 76). ${ }^{130}$ This free radical multi-component reaction consists in adding 2 equiv. of $\mathrm{Mn}(\mathrm{OAc})_{3}$ to the three substrates in DMSO at $80{ }^{\circ} \mathrm{C}$. The reaction accepts a broad scope of anilines furnishing 2pyrrolines in good to excellent yields irrespective of the electronic nature or position of substituent. Regarding the alkenes 239, the methodology displays a broad scope for terminal olefins with yields ranging from $60 \%$ to $91 \%$. Alkyl amines and 1,2 disubstituted alkenes fails to delivers the 2-pyrrolines.

2.2.6. Synthesis of 2-pyrrolines by nickel catalysis. France et al. ${ }^{131}$ developed a general synthetic approach to 4-carboxyand 4-keto-2,3-dihydropyrroles 242 using $\mathrm{Ni}\left(\mathrm{ClO}_{4}\right)_{2} \cdot 6 \mathrm{H}_{2} \mathrm{O}$ as
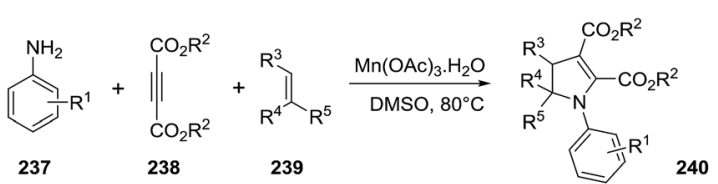

240a, $R^{1}=\mathrm{H} ; \mathrm{R}^{2}=\mathrm{Et} ; \mathrm{R}^{3}=\mathrm{H} ; \mathrm{R}^{4}=\mathrm{H} ; \mathrm{R}^{5}=\mathrm{Ph} ; 85 \%$ 240b, $R^{1}=4-M e ; R^{2}=E t ; R^{3}=H ; R^{4}=H ; R^{5}=P h ; 85 \%$ 240c, $R^{1}=4-\mathrm{MeO} ; R^{2}=E t ; R^{3}=H ; R^{4}=H ; R^{5}=\mathrm{Ph} ; 79 \%$ 240d, $R^{1}=4-\mathrm{Cl} ; \mathrm{R}^{2}=\mathrm{Et} ; \mathrm{R}^{3}=\mathrm{H} ; \mathrm{R}^{4}=\mathrm{H} ; \mathrm{R}^{5}=\mathrm{Ph} ; 78 \%$ 240e, $R^{1}=4-B r ; R^{2}=E t ; R^{3}=H ; R^{4}=H ; R^{5}=P h ; 76 \%$ 240f, $R^{1}=3-M e ; R^{2}=E t ; R^{3}=H ; R^{4}=H ; R^{5}=P h ; 84 \%$ 240g, $\mathrm{R}^{1}=3-\mathrm{NO}_{2} ; \mathrm{R}^{2}=\mathrm{Et} ; \mathrm{R}^{3}=\mathrm{H} ; \mathrm{R}^{4}=\mathrm{H} ; \mathrm{R}^{5}=\mathrm{Ph} ; 60 \%$ 240h, R $\mathrm{R}^{1}=2-\mathrm{Me} ; \mathrm{R}^{2}=\mathrm{Et} ; \mathrm{R}^{3}=\mathrm{H} ; \mathrm{R}^{4}=\mathrm{H} ; \mathrm{R}^{5}=\mathrm{Ph} ; 82 \%$ 240i, $R^{1}=H ; R^{2}=M e ; R^{3}=H ; R^{4}=H ; R^{5}=P h ; 84 \%$. 240j, $R^{1}=H ; R^{2}=E t ; R^{3}=H ; R^{4}=H ; R^{5}=4-M_{e} C_{6} H_{4} ; 84 \%$ 240k, $R^{1}=\mathrm{H} ; \mathrm{R}^{2}=\mathrm{Et} ; \mathrm{R}^{3}=\mathrm{H} ; \mathrm{R}^{4}=\mathrm{H} ; \mathrm{R}^{5}=4-t-\mathrm{BuC}_{6} \mathrm{H}_{4} ; 79 \%$ 240I, $R^{1}=H ; R^{2}=E t ; R^{3}=H ; R^{4}=H ; R^{5}=4-F_{6} H_{4} ; 80 \%$ 240m, $\mathrm{R}^{1}=\mathrm{H} ; \mathrm{R}^{2}=\mathrm{Et} ; \mathrm{R}^{3}=\mathrm{H} ; \mathrm{R}^{4}=\mathrm{H} ; \mathrm{R}^{5}=4-\mathrm{ClC}_{6} \mathrm{H}_{4} ; 83 \%$ 240n, $R^{1}=H ; R^{2}=E t ; R^{3}=H ; R^{4}=H ; R^{5}=4-B C_{6} H_{4} ; 75 \%$

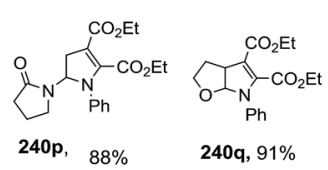
240o, $R^{1}=H ; R^{2}=E t ; R^{3}=H ; R^{4}=M e ; R^{5}=P h ; 87 \%$

Scheme 76 Synthesis of polysubstituted 2-pyrrolines 240 

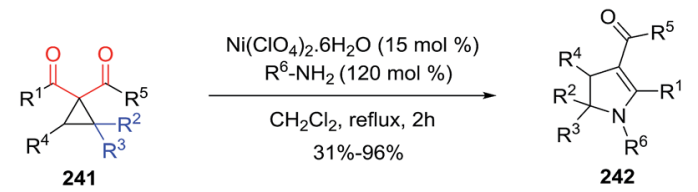

Scheme 77 Amine ring-opening cyclizations of cyclopropanes to form 2-pyrolines. Donor moiety in blue, acceptor moiety in red.

a Lewis acid catalyst via nucleophilic primary amine ringopening cyclizations of donor-acceptor (D-A) cyclopropanes 241 (Scheme 77). The optimized conditions were $15 \mathrm{~mol} \%$ of $\mathrm{Ni}\left(\mathrm{ClO}_{4}\right)_{2} \cdot 6 \mathrm{H}_{2} \mathrm{O}$ with 1.2 equiv. of the primary amine in refluxing $\mathrm{CH}_{2} \mathrm{Cl}_{2}$ or 1,2-dichloroethane. In this way, several primary amine nucleophiles and different substituted D-A cyclopropanes 241 provided highly substituted 2,3-dihydropyrroles $\mathbf{2 4 2}$ with an electron-withdrawing group in position four in good to excellent yields (31-96\%). The particularity of 2,3 dihydropyrroles bearing an electron-withdrawing group at position four is the extended conjugation with the enamine that makes possible vinylogous reactivity.

Taking advantage of the fact that the synthesis of the D-A cyclopropanes $\mathbf{2 4 1}$ from alkenes $\mathbf{2 4 4}$ and $\alpha$-diazo carbonyls 243 and the subsequent pyrroline formation are both carried out in $\mathrm{CH}_{2} \mathrm{Cl}_{2}$, the authors performed the tandem one-pot cyclopropanation/amine ring-opening cyclization to obtain the desired vinylogous 2,3-dihydropyrroles 242 in very good yield for the two steps (Scheme 78).
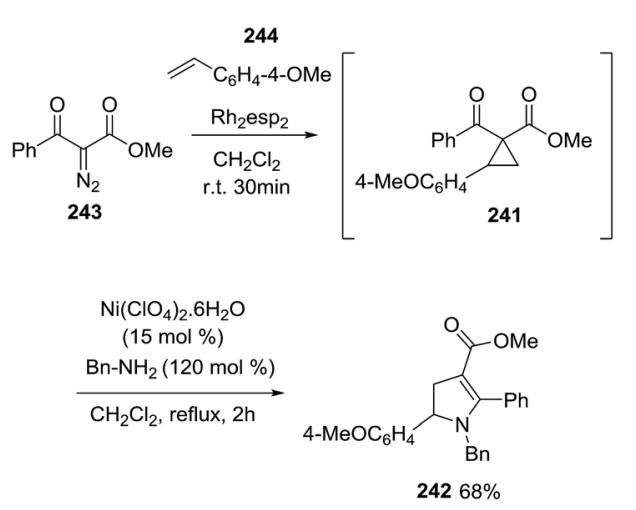

Scheme 78 One-pot tandem cyclopropanation/amine ring-opening cyclization.

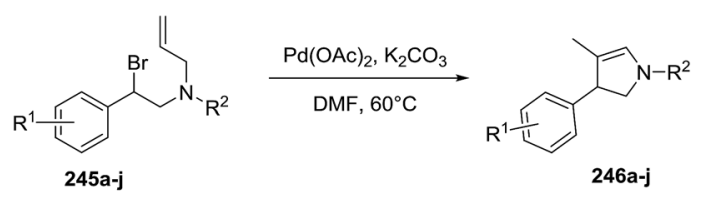

246a, $R^{1}=H ; R^{2}=$ Ts $86 \%$
246b, $R^{1}=p-C l ; R^{2}=$ Ts $83 \%$
246c, $R^{1}=m-C l ; R^{2}=$ Ts $63 \%$
246d, $R^{1}=o-C l ; R^{2}=$ Ts $43 \%$
246e, $R^{1}=p-M e ; R^{2}=$ Ts $67 \%$
246f, $R^{1}=p-A c O ; R^{2}=$ Ts $52 \%$

246g, $R^{1}=p-t-B u ; R^{2}=T s 76 \%$ 246h, $R^{1}=p-C N ; R^{2}=T s, 91 \%$ 246i, $\mathrm{R}^{1}=\mathrm{H} ; \mathrm{R}^{2}=\mathrm{SO}_{2} \mathrm{Ph} 76 \%$ 246j, $\mathrm{R}^{1}=p-t-\mathrm{Bu} ; \mathrm{R}^{2}=\mathrm{SO}_{2} \mathrm{Ph}, 95 \%$

Scheme 79 Palladium-catalyzed intramolecular Heck reaction towards 2-pyrrolines.

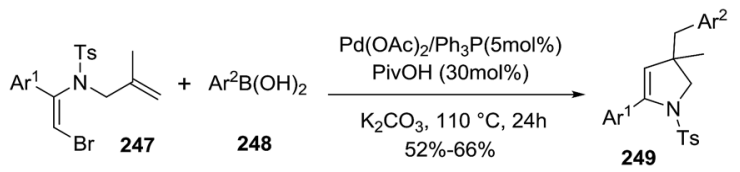

Scheme 80 Palladium-catalyzed tandem synthesis of 2-pyrrolines.

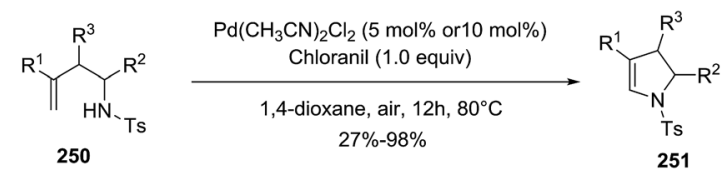

$\mathbf{R}^{1}=$ Aryl, COOR

$\mathrm{R}^{2}=$ (Hetero)Aryl, Alkyl, Alkenyl

$\mathrm{R}^{3}=\mathrm{H}, \mathrm{Me}$

Scheme $81 \mathrm{Pd}$-promoted intramolecular oxidative amination towards 2-pyrrolines.

2.2.7. Synthesis of 2-pyrrolines by palladium catalysis. A broad variety of approaches towards the synthesis of 2-pyrrolines using palladium catalysts has been developed. Several of these involve the cyclization of alkenyl-substituted amines. In particular, bromoalkenyl amines provide the 2-pyrroline via Pdcatalysis of the intramolecular Heck reaction. For instance, Pan et al. ${ }^{\mathbf{1 3 2}}$ described a ligand-free palladium catalyst for the cyclization of secondary benzylic bromides bearing $\beta$-hydrogens 245 (Scheme 79). The optimal reaction conditions were $\mathrm{Pd}(\mathrm{OAc})_{2}$ $(1 \mathrm{~mol} \%)$ and $\mathrm{K}_{2} \mathrm{CO}_{3}$ in DMF at $60{ }^{\circ} \mathrm{C}$ for $24 \mathrm{~h}$. The desired 2pyrrolines $\mathbf{2 4 6}$ were obtained with moderate to excellent yields and with high regioselectivities. Suitable substrates included different aromatic substituents, as well as various $\mathrm{N}$-sulfonyl amines.

A variety of 2-pyrrolines $\mathbf{2 4 9}$ derivatives were synthesized from the reaction of vinyl bromide 247 with arylboronic acids 248 by a palladium-catalyzed tandem intramolecular Heck/ intermolecular Suzuki cross-coupling reaction (Scheme 80). ${ }^{\mathbf{1 3 3}}$ The optimized conditions were $5 \mathrm{~mol} \%$ of $\mathrm{Pd}(\mathrm{OAc})_{2}, 5 \mathrm{~mol} \%$ of $\mathrm{PPh}_{3}, 2$ equiv. $\mathrm{K}_{2} \mathrm{CO}_{3}$ and $30 \mathrm{~mol} \%$ of pivalic acid in $N, N-$ dimethylacetamide at $110{ }^{\circ} \mathrm{C}$ for 24 hours. The 2-pyrrolines 249 were obtained in moderate to good yields (typically 52-66\%).

Loh et al. ${ }^{\mathbf{1 3 4}}$ described the use of $N$-homoallyl- $N$-tosyl amines 250 to generate multisubstituted dihydropyrroles 251 via direct amination of the alkene (Scheme 81). The optimal reaction conditions of this oxidative amination used $\mathrm{Pd}\left(\mathrm{CH}_{3} \mathrm{CN}\right)_{2} \mathrm{Cl}_{2}$ (10 mol\%) and chloranil (1 equiv.) as the oxidant in 1,4-dioxane, at $80{ }^{\circ} \mathrm{C}$ for $24 \mathrm{~h}$ under air atmosphere. Different derivatizations at several positions $\left(\mathrm{R}^{1}-\mathrm{R}^{3}\right)$ achieved the pyrroline with

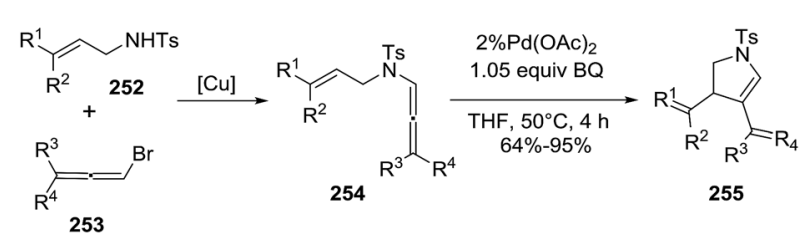

Scheme 82 Carbocyclization of aza-enallenes 254 promoted by [Pd]. 


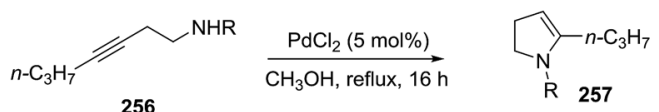

$256257 a, R=T s(61 \%)$

257a, R=Ts (Microwave-assisted heating, $70^{\circ} \mathrm{C}, 20 \mathrm{~min}, 85 \%$ )

257a, R=Ts (AuCl, $5 \mathrm{~mol} \%, 85 \%$ )

257b, R=Ms (36\%)

257c, $\mathrm{R}=$ Boc (solvent $\mathrm{CH}_{2} \mathrm{Cl}_{2}, 28 \%$ )

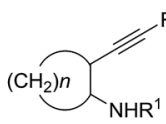

$R^{2}$ $\mathrm{PdCl}_{2} 5 \mathrm{~mol} \%, \mathrm{CH}_{3} \mathrm{CN}$
$\begin{gathered}\text { Microwave-assisted heating } \\ 85^{\circ} \mathrm{C}, 20 \mathrm{~min}\end{gathered}$

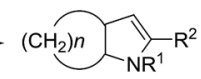

258: $n=3 ; n=4$

259 259m, $n=3, \mathrm{R}^{1}=\mathrm{Ts}, \mathrm{R}^{2}=2-\mathrm{FC}_{6} \mathrm{H}_{4}, 74 \%$ 259n, $n=4, \mathrm{R}^{1}=\mathrm{Ts}, \mathrm{R}^{2}=\mathrm{Ph}, 39 \%$ 259o, $n=4, \mathrm{R}^{1}=\mathrm{Ts}, \mathrm{R}^{2}=\mathrm{Ph}, 60 \%{ }^{\mathrm{a}}$ 259p, $n=4, \mathrm{R}^{1}=\mathrm{Ts}, \mathrm{R}^{2}=n-\mathrm{Bu}, 47 \%^{\mathrm{a}}$ 259q, $n=4, \mathrm{R}^{1}=\mathrm{Ts}, \mathrm{R}^{2}=4-\mathrm{NO}_{2} \mathrm{C}_{6} \mathrm{H}_{4}, 51 \%^{\mathrm{a}}$ 259r, $n=4, \mathrm{R}^{1}=\mathrm{Ts}, \mathrm{R}^{2}=4-\mathrm{CO}_{2} \mathrm{MeC}_{6} \mathrm{H}_{4}, 50 \%$ a 259s, $n=4, \mathrm{R}^{1}=\mathrm{Ts}, \mathrm{R}^{2}=4-\mathrm{ClC}_{6} \mathrm{H}_{4}, 57 \%{ }^{\mathrm{a}}$ 259t, $n=4, \mathrm{R}^{1}=\mathrm{Ts}, \mathrm{R}^{2}=4-\mathrm{MeC}_{6} \mathrm{H}_{4}, 64 \%{ }^{\mathrm{a}}$ 259u, $n=4, \mathrm{R}^{1}=\mathrm{Ts}, \mathrm{R}^{2}=4-\mathrm{t}-\mathrm{BuC}_{6} \mathrm{H}_{4}, 55 \%{ }^{\mathrm{a}}$ 259v, $n=4, \mathrm{R}^{1}=\mathrm{Ts}, \mathrm{R}^{2}=4-\mathrm{MeOC}_{6} \mathrm{H}_{4}, 42 \%{ }^{\mathrm{a}}$

a $\mathrm{AuCl}$ instead of $\mathrm{PdCl}_{2}$

Scheme 83 Pd-catalyzed intramolecular hydroamination of $\mathrm{N}$-(3butynyl) sulfonamides towards mono and bicyclic 2-pyrrolines.

moderate to high yield (27-98\%), proving the versatility of the reaction. Notably, the 5-endo-trig cyclization proceeded efficiently when the alkenes were substituted with electronwithdrawing groups at $\mathrm{R}^{1}$ position. On the contrary, when $\mathrm{R}^{1}$ is a long alkyl chain the annulations did not take place.

Bäckvall reported the carbocyclization of aza-enallenes 254 in the presence of catalytic $\mathrm{Pd}(\mathrm{OAc})_{2}$ to give the 2-pyrrolines 255 (Scheme 82). ${ }^{\mathbf{1 3 5}}$ Several substitution patterns on both the alkene 252 and allene 253 were well tolerated, providing moderate to high yields of the final pyrrolines (64-95\%) (Scheme 82).

Hou et al. ${ }^{125}$ studied the Pd-catalyzed intramolecular hydroamination of $\mathrm{N}$-(3-butynyl) sulfonamides leading to 2,3-dihydropyrroles (Scheme 83). Both $N$-substituted 3-heptynamines 256 and the cyclopentanamine (and cyclohexanamine) analogues 258 underwent 5-endo-dig cyclization providing 5propyl-2,3-dihydro- $1 H$-pyrroles $257 \mathbf{a}-\mathbf{c}$ and $259 \mathbf{a}-\mathbf{v}$, respectively, with good yields (up to $92 \%$ ). The optimal reaction conditions for 256 used $\mathrm{PdCl}_{2}(5 \mathrm{~mol} \%)$ in anhydrous methanol at reflux for

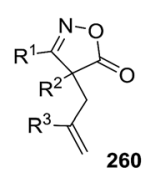

$\left[\mathrm{Pd}_{2}(\mathrm{dba})_{3}\right](2.5 \mathrm{~mol} \%)$ $\mathrm{P}\left(4-\mathrm{CF}_{3} \mathrm{C}_{6} \mathrm{H}_{4}\right)_{3}(10 \mathrm{~mol} \%)$

dioxane, $80^{\circ} \mathrm{C}, 12 \mathrm{~h}$

260

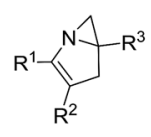

261 261a, $R^{1}=\mathrm{Ph}, \mathrm{R}^{2}=\mathrm{Me}, \mathrm{R}^{3}=\mathrm{Me}, 87 \%$ 261b, $R^{1}=2$-naphthyl, $R^{2}=M e, R^{3}=M e, 83 \%$ 261c, $R^{1}=4-\mathrm{CF}_{3} \mathrm{C}_{6} \mathrm{H}_{4}, \mathrm{R}^{2}=\mathrm{Me}, \mathrm{R}^{3}=\mathrm{Me}, 80 \%{ }^{[\mathrm{a}]}$ 261d, $R^{1}=P h, R^{2}=P h, R^{3}=M e, 88 \%{ }^{[a]}$ 261e, $\mathrm{R}^{1}=\mathrm{Ph}, \mathrm{R}^{2}=\mathrm{Me}, \mathrm{R}^{3}=n$-hexyl, $80 \%$ 261f, $\mathrm{R}^{1}=n \operatorname{Pr}, \mathrm{R}^{2}=\mathrm{Me}, \mathrm{R}^{3}=n$-hexyl, $63 \%{ }^{[\mathrm{a}]}$ 261g, $R^{1}=M e, R^{2}=B n, R^{3}=M e, 64 \%[a]$ 261h, $R^{1}=\mathrm{Me}, \mathrm{R}^{2}=$ methallyl, $\mathrm{R}^{3}=\mathrm{Me}, 40 \%$ [a] 261i, (65\%)

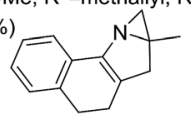

[a] $\left[\mathrm{Pd}_{2}(\mathrm{dba})_{3}\right](5 \mathrm{~mol} \%)$, and $\mathrm{P}\left(4-\mathrm{CF}_{3} \mathrm{C}_{6} \mathrm{H}_{4}\right)_{3}(20 \mathrm{~mol} \%)$ were used.

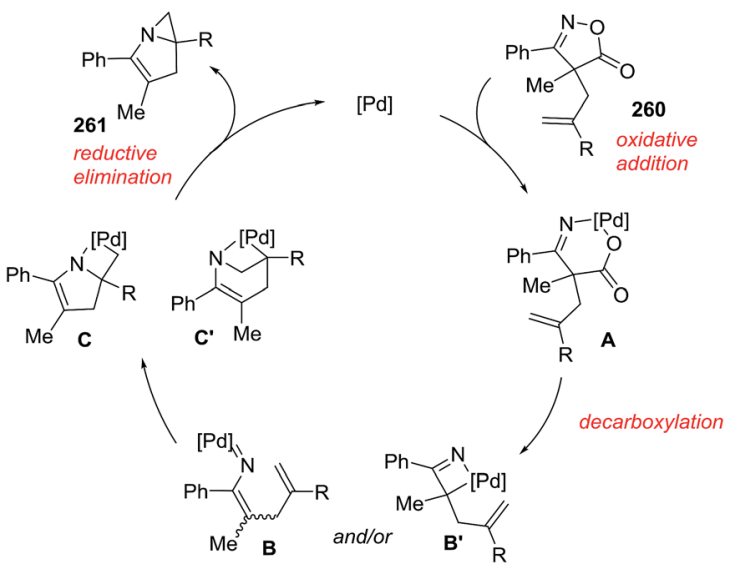

Scheme 85 Proposed mechanism for the intramolecular aziridation transformation.

16 hours. In contrast, the amines 258 required microwave heating in acetonitrile for 20 minutes to achieve the bicyclic-2pyrrolines 259 with higher yields. Optimum results were obtained with aryl-alkynes substituted with electron-withdrawing groups on the aromatic ring $\left(\mathrm{R}^{2}=4-\mathrm{NO}_{2}-\mathrm{Ph}, 3-\mathrm{Cl}-\mathrm{Ph}\right)$. Interestingly, the annulation of 256 into 257 and 258 into 259 proceeded efficiently in presence of $\mathrm{AuCl}$ catalyst.

Okamoto et al. developed the Pd-catalyzed intramolecular aziridination of $4 \mathrm{H}$-isoxazol-5-ones 260 leading to $\mathrm{N}$-fused bicyclic aziridines $\mathbf{2 6 1 a - i}$ (Scheme 84) as a different approach. ${ }^{\mathbf{1 3 6}}$ The optimal reaction conditions used $\operatorname{Pd}_{2}(\mathrm{dba})_{3}(2.5 \mathrm{~mol} \%)$ and $\left(4-\mathrm{CF}_{3} \mathrm{C}_{6} \mathrm{H}_{4}\right)_{3} \mathrm{P}(10 \mathrm{~mol} \%)$ in dioxane at $80{ }^{\circ} \mathrm{C}$ for 12 hours, affording the fused bicyclic structures in good yields (63-88\%). Aromatic substituents at $\mathrm{R}^{1}$ position proved to be very reactive substrates. Furthermore, the tetracyclic product 261i was obtained in $65 \%$ yield. The proposed mechanism starts with an oxidative addition of isoxazolone 260 leading to a six-membered palladacycle $\mathbf{A}$, which readily undergoes decarboxylation towards vinylnitrene/palladium complex $\mathbf{B}$ and/or fourmembered azapalladacyclobutene intermediate $\mathbf{B}^{\prime}$ (Scheme 85). Subsequent cycloaddition of these alkenes provides two possible azapalladacycles $\mathbf{C}$ and $\mathbf{C}^{\prime}$, which can undergo the reductive elimination to produce bicyclic aziridine 261 .

Palladium-mediated intermolecular strategies affording 2pyrrolines were developed as well. As an example, Yoshida et al. prepared the 2-vinyl-2,3-dihydropyrroles 264 from reaction of the $\beta$-enaminocarbonyls 262 with 1,4-diacetoxy-2-butene 263, using $\mathrm{Pd}(\mathrm{OAc})_{2}$ as catalyst and BINAP as an additive (Scheme 86). ${ }^{137}$ The reaction tolerates a variety of sulfonyl groups as the EWG moiety on nitrogen, and as well different alkyl and aryl

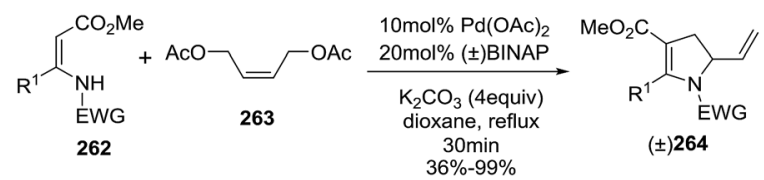

Scheme 86 Synthesis of functionalized 2-pyrrolines by oxidative radical cyclization of $N$-sulfonyl $\beta$-enamino esters with alkenes. 
<smiles>[R]ON1C([R]O)N2C=CC21</smiles>

265

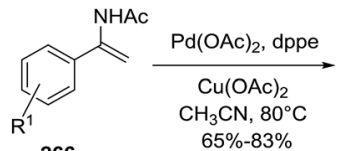

266

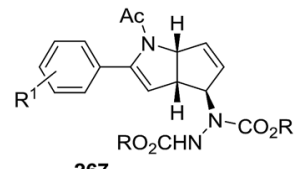

267
Scheme $87 \mathrm{Pd}$-catalyzed $\mathrm{CH}$ activation/oxidative coupling towards cyclopentene fused 2-pyrrolines.

substituents on the $\beta$-enamino ester. In addition, both $E$ or $Z$ 2butene-1,4-diol bisacetates can be used to obtain 2-vinyl-2,3dihydropyrroles 264.

A second example of Pd-catalyzed $\mathrm{C}-\mathrm{H}$ activation/oxidative is the coupling of enamides 266 with diazabicyclic olefins 265 , leading to the cyclopentane-fused N-protected 2-pyrrolines 267 (Scheme 87). ${ }^{138}$ The optimal reaction conditions (using 265 with enamide 266) were $\mathrm{Pd}(\mathrm{OAc})_{2}$ (10 mol\%), $\mathrm{Cu}(\mathrm{OAc})_{2}$ (2.0 equiv.), and dppe $(10 \mathrm{~mol} \%)$ in acetonitrile at $80{ }^{\circ} \mathrm{C}$ for 12 hours. In general, aryl enamides with electron-withdrawing substituents on the aromatic ring afforded the highest yields (65-83\%). A plausible mechanism would include two stages (Scheme 88).

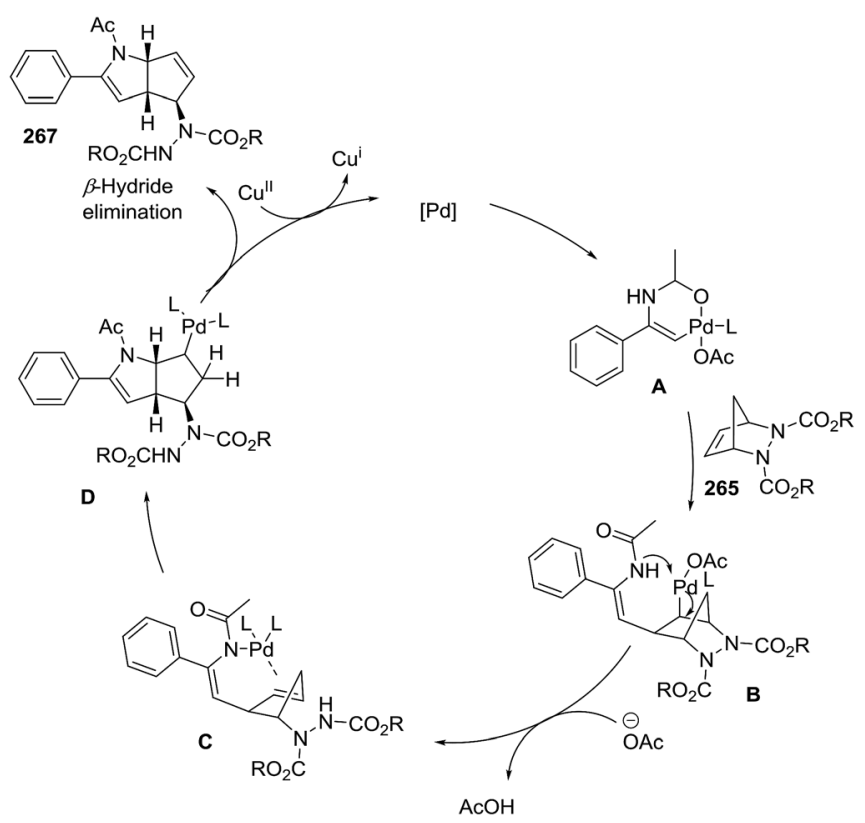

Scheme 88 Proposed mechanism for the Pd-catalyzed $\mathrm{CH}$ activation/oxidative coupling.

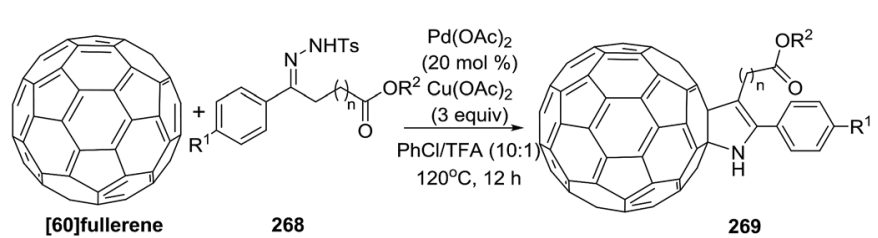

269a, $\mathrm{R}^{1}=\mathrm{H} ; \mathrm{R}^{2}=\mathrm{Me} ; \mathrm{n}=2 ; 28 \%$ 269b, $R^{1}=H ; R^{2}=E t ; n=2 ; 30 \%$ 269c, $\mathrm{R}^{1}=\mathrm{H} ; \mathrm{R}^{2}=i-\mathrm{Pr} ; \mathrm{n}=2 ; 34 \%$ 269d, $R^{1}=H ; R^{2}=M e ; n=1 ; 24 \%$ 269e, $R^{1}=H ; R^{2}=E t ; n=1 ; 27 \%$ 269f, $R^{1}=O M e ; R^{2}=M e ; n=2 ; 31 \%$

269g, $R^{1}=H ; R^{2}=$ sec - Butyl; $n=2 ; 43 \%$ 269h, $R^{1}=H ; R^{2}=$ Phenethyl; $n=2 ; 37 \%$ 269i, $R^{1}=H ; R^{2}=B u ; n=2 ; 31 \%$ 269j, $R^{1}=H ; R^{2}=\operatorname{Pr} ; n=2 ; 28 \%$ 269k, $R^{1}=F ; R^{2}=M e ; n=2 ; 21 \%$ 269, $R^{1}=F ; R^{2}=E t ; n=2 ; 24 \%$

Scheme $89 \mathrm{Pd}$-catalyzed syntheses of the fulleropyrrolines 269 .

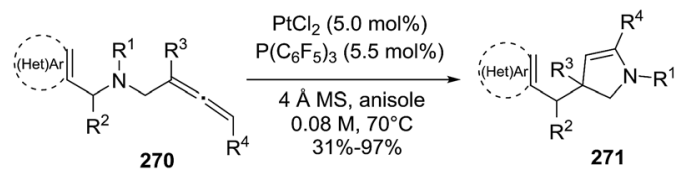

Scheme 90 Platinum-catalyzed intramolecular annulation of (hetero) aryl-allenes.

The first is $\mathrm{C}-\mathrm{H}$ bond activation of the enamide 266 by $\mathrm{Pd}(\mathrm{OAc})_{2}$ to form cyclic intermediate A. Coordination of the alkene 265 to A followed by carbopalladation would produce intermediate $\mathbf{B}$. Aminopalladation and subsequent ring opening of $\mathbf{A}$ could generate intermediate $\mathbf{C}$, which would furnish the bicyclic product 267 via a final $\beta$-hydride elimination. Reoxidation of palladium by the $\mathrm{Cu}(\mathrm{II})$ completes the catalytic cycle.

Peng et al. reported an efficient Pd-catalyzed synthesis of the scarce $N$-unsubstituted 2-fulleropyrrolines 269 employing [60] fullerene and benzoyl hydrazone esters 268 (Scheme 89). ${ }^{139}$ The reaction involves the use of $20 \mathrm{~mol} \%$ of $\mathrm{Pd}(\mathrm{OAc})_{2}$ and 3 equivalent of $\mathrm{Cu}(\mathrm{OAc})_{2}$ as oxidant in a solvent mixture of chlorobenzene/TFA $(10: 1)$ at $120{ }^{\circ} \mathrm{C}$. The presence of TFA generates a highly electrophilic cationic Pd(II) species and provides better results. The reaction of benzoyl hydrazones esters 268 with electron-donating or electron-withdrawing substituent on the phenyl ring and a variety of esters proceeded efficiently in acceptable yields (21-43\%).

2.2.8. Synthesis of 2-pyrrolines by platinum catalysis. A new synthesis of 2-pyrrolines was recently developed by Shi et al. via platinum-catalyzed cyclization of a variety of (hetero)arylallenes 270, through the migration of the (hetero)arylmethylene group (Scheme 90). ${ }^{140}$ After screening several catalysts across a number of conditions, the use of $\mathrm{PtCl}_{2}(5 \mathrm{~mol} \%)$ with an electron-deficient phosphine ligand such as $\mathrm{P}\left(\mathrm{C}_{6} \mathrm{~F}_{5}\right)_{3}$ in anisole at $70{ }^{\circ} \mathrm{C}$ were identified as the optimal conditions. This $\mathrm{Pt}(\mathrm{II})$-catalyzed migration protocol tolerates a wide range of (hetero)aryl-allenes $\mathbf{2 7 0}$. The desired 2-pyrrolines 271 are obtained in yields up to $97 \%$.

2.2.9. Synthesis of 2-pyrrolines by rhenium catalysis. Kusama et al. ${ }^{141}$ reported the $\left[\mathrm{ReCl}(\mathrm{CO})_{5}\right]$-catalyzed $(10 \mathrm{~mol} \%)$ synthesis of the 2-azabicyclo[3.3.0]octane 273 and the allenylsubstituted dihydropyrrole 274, from the sulfonamide $\mathbf{2 7 2}$ (Scheme 91). This transformation can also be carried out by tungsten(0) catalysis. By evaluating different sulfonyl groups and alternative rhenium catalysts, the selective preparation of either $\mathbf{2 7 3}$ or $\mathbf{2 7 4}$ was possible. The optimal conditions for $\mathbf{2 7 3}$ used an $N$-nosyl amine and $\left[\operatorname{ReCl}(\mathrm{CO})_{4} \cdot \mathrm{PPh}_{3}\right]$ as a neutral

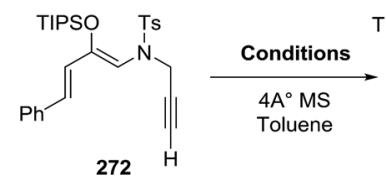
$10 \mathrm{~mol} \%\left[\operatorname{ReCl}(\mathrm{CO})_{5}\right], 80^{\circ} \mathrm{C}, 0.5 \mathrm{~h}$
$10 \mathrm{~mol} \%$ [W(CO)6], $h v, 1.5 \mathrm{~h}$
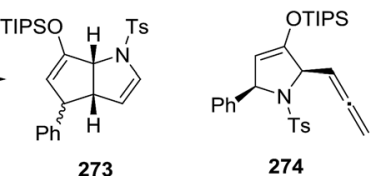

$68 \%(\alpha / \beta=67: 33)$

$46 \%(\alpha / \beta=90: 10) \quad 43 \%$ (single isomer)
Scheme 91 Tungsten(0)- and rhenium(I)-catalyzed tandem cyclization of acetylenic dienol silyl ethers. 


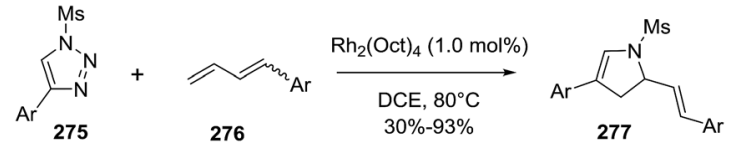

Scheme 92 Aza- $[4+3]$ annulation through sequential $[3+2]-[2+1]$ cycloadditions leading to 2-pyrrolines.
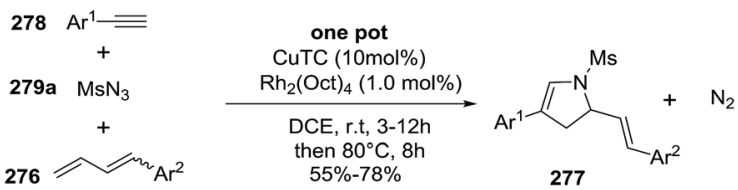

Scheme 93 One-pot synthesis of 2-pyrrolines starting from arylacetylenes, mesyl azide, and buta-1,3-dienylbenzenes.

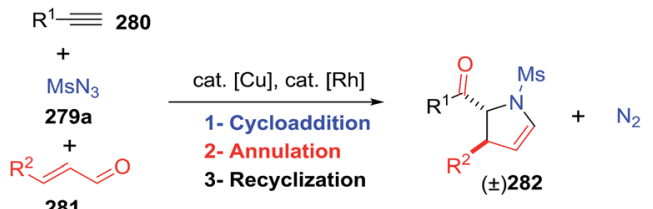

Scheme 94 Diastereoselective synthesis of trans-2-pyrrolines starting from terminal alkynes, $N$-sulfonyl azides, and $\alpha, \beta$-enals.

catalyst. On the other hand, dihydropyrrole 274 was obtained as the major product with the $p$-methoxybenzenesulfonyl (Mbs) sulfonamide and the cationic rhenium catalyst prepared in situ from $\left[\mathrm{ReCl}(\mathrm{CO})_{5}\right](1 \mathrm{~mol} \%)$ and $\mathrm{AgSbF}_{6}(10 \mathrm{~mol} \%)$. These reactions demonstrate the high synthetic utility of geminal carbofunctionalization of alkynes and rhenium and tungsten complexes in pyrroline synthesis.

2.2.10. Synthesis of 2-pyrrolines by rhodium catalysis. Kim et al. reported the straightforward synthesis of 2-pyrrolines 277 from sulfonyl triazoles 275 and 1,3-dienes 276, catalyzed by $\mathrm{Rh}_{2}(\mathrm{Oct})_{4}$, under liberation of $\mathrm{N}_{2}$ as the single byproduct (Scheme 92). This product results from sequential [3+2]-[2+1] cycloadditions giving a net $[4+3]$ aza-annulation. ${ }^{142}$ The sulfonyl group structure affected appreciably the yield of the reaction. The $N^{1}$-methanesulfonyl triazole was best, while a tosyl group was not tolerated. In contrast, different substitu-

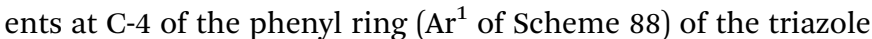
did not affect the yield. Both the $Z$ - or $E-1,3$ diene gave similar yields of 277. Finally, the authors applied this procedure starting from terminal alkynes 278, mesyl azide, and 1,3-dienes 276 (Scheme 93). This one-pot, three-component synthesis of dihydropyrroles is a synthetically attractive annulation. In the first step, the mesyl triazole $\mathbf{2 7 5}$ is generated through a coppercatalyzed click reaction between the terminal alkyne $\mathbf{2 7 8}$ and the mesyl azide 279. Rhodium-catalyzed annulation then leads to the 2-pyrrolines 277. This result demonstrates that the copper does not interfere with this final annulation.

A rhodium(II)-catalyzed cycloaddition of 1-sulfonyl 1,2,3-triazoles 283 with $\alpha, \beta$-unsaturated aldehydes 281 was disclosed in 2013 by the Murakami group. ${ }^{143}$ The combination of terminal

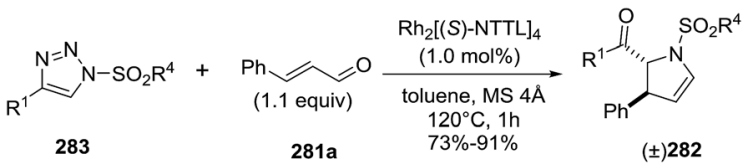

Scheme $95 \mathrm{Rh}($ (I)-catalyzed denitrogenative annulation of triazoles to synthesize racemic trans-2,3-disubstituted 2-pyrrolines.

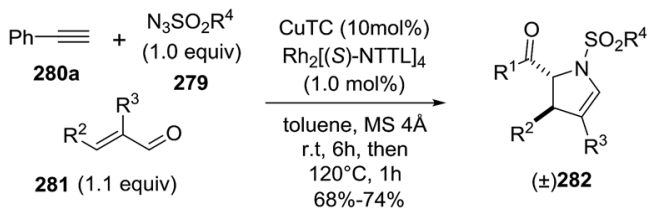

Scheme 96 One-pot synthesis of 2-pyrrolines 282 starting from phenylacetylene.

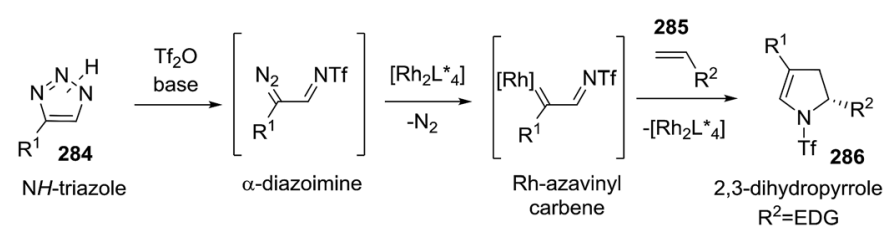

Scheme 97 Reactions of N-triflyl-Rh-azavinyl carbenes with olefins.

alkynes 280, $N$-sulfonyl azides 279 , and $\alpha, \beta$-unsaturated aldehydes $\mathbf{2 8 1}$ as starting materials resulted in the diasteroselective formation of the racemic trans-2,3-disubstituted 2-pyrroline $\mathbf{2 8 2}$ (Scheme 94).

$\mathrm{N}$-Sulfonyl-1,2,3-triazoles $\mathbf{2 8 3}$ are useful intermediates in the synthesis of heterocycles. In the presence of rhodium catalysts these triazoles generate $\alpha$-imino rhodium carbene (azavinylcarbenes) which reacts with the $\alpha, \beta$-unsaturated aldehydes 281 to give the 2-pyrrolines 282 (Scheme 95). With the chiral and bulky $\left[\mathrm{Rh}_{2}(\mathrm{~S}-\mathrm{NTTL})_{4}\right]$ as the catalyst, the exclusive formation of the racemic trans-2,3 disubstituted 2-pyrroline is observed. With less bulky Rh catalysts, an undesired 4,5-dihydro-1,4oxazepine subproduct is observed. The production of the racemic dihydropyrroles even though the use of a chiral catalyst is explained by a non-stereospecific ionic mechanism. A screening of $\alpha, \beta$-unsaturated aldehydes 281 revealed that a variety of $(E)$ and $(Z) \beta$-monosubstituted enals and acyclic $\alpha, \beta$ disubstituted enals were converted effectively into the 2-pyrrolines. Different groups at the 4-position of triazoles 283 and on the sulfonyl group all participated in the annulation reaction to give good yields (typically 80\%).

The reaction was made more practical in the form of a onepot synthesis starting from terminal alkynes 280a, sulfonyl azides $\mathbf{2 7 9}$, and $\alpha, \beta$-unsaturated aldehydes 281 , using a mixture of CuTC $(10 \mathrm{~mol} \%)$ and $\mathrm{Rh}_{2}[(\mathrm{~S})-\mathrm{NTTL}]_{4}(1.0 \mathrm{~mol} \%)$ as cocatalysts (Scheme 96).

Using a similar strategy the Fokin group synthesized 2-pyrrolines 286 from in situ generated $N$-triflyl triazoles 284 and alkenes 285 (Scheme 97). ${ }^{144}$ Azavinylcarbenes can be obtained directly from $N$-sulfonyl triazoles or $N$-triflyltriazoles in presence 


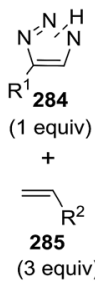

1- $\left[\mathrm{Rh}_{2}(\mathrm{~S}-\mathrm{NTTL})_{4}\right](0.5 \mathrm{~mol} \%)$ DTBMP (1.2 equiv) $\stackrel{\text { anhydrous } \mathrm{CHCl}_{3}(0.17 \mathrm{M})}{\longrightarrow}$

2- $\mathrm{Tf}_{2} \mathrm{O}$ (1.5 equiv) $-30^{\circ} \mathrm{C}$ to rt $32 \%-84 \%$ $15 \%-74 \%$ ee

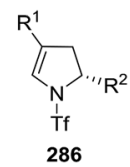

(3 equiv)

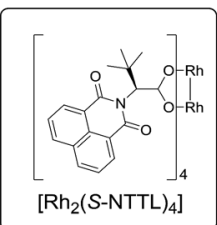

2,6-di-tert-butyl-4-methylpyridine (DTBMP)

Scheme 98 Catalytic asymmetric transannulation of $\mathrm{NH}-1,2,3$-triazoles with alkenes.

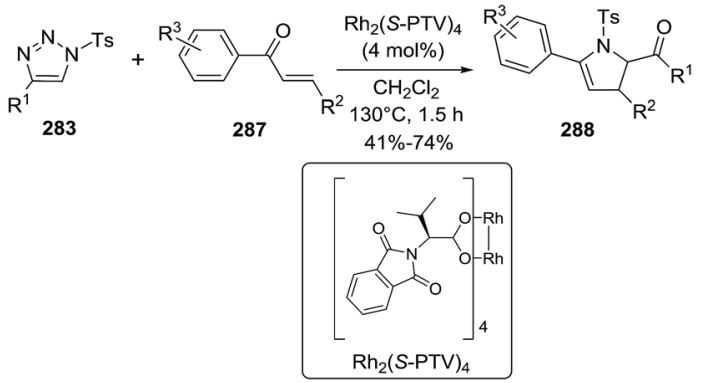

Scheme 99 Carbenoid strategy to generate multisubstituted 2pyrrolines.

of a rhodium catalyst. These azavinyl carbenes react with 4methoxystyrene to produce enantioenriched 2-pyrrolines 286 when a chiral rhodium catalyst is used. The highest enantioselectivity $\left(72 \%\right.$ ee) was achieved with $\left[\mathrm{Rh}_{2}(\mathrm{~S}-\mathrm{NTTL})_{4}\right]$ (Scheme 98). The authors suggest that the moderate enantioselectivity arises from a rapid bond rotation that erodes the enantioselectivity during the mechanism. The protocol affords 2-pyrrolines when electron-rich olefins such as 4-methoxystyrene or 2methoxystyrene are used as partners.

An efficient Rh-catalyzed annulation between $\alpha, \beta$-unsaturated ketones 287 and $N$-sulfonyl-1,2,3-triazoles 283 have been developed leading to multisubstituted 2-pyrrolines 288 (Scheme 99). ${ }^{145}$ In this methodology, the generated $\alpha$-rhodium imino carbene species served as the electrophiles against the $\alpha, \beta$ unsaturated ketones, which produced a nucleophilic attack through their oxygen atom. Interestingly, small structural

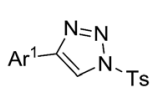

283

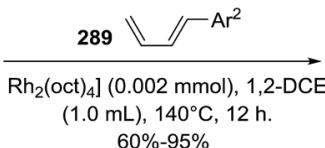

$60 \%-95 \%$

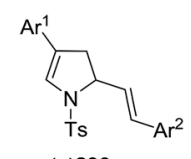

$( \pm) 290$
Scheme 100 Rhodium(II)-catalyzed formal aza-[3 + 2] cycloadditions of 1-sulfonyl 1,2,3-triazoles with (E)-1-aryl-1,3-butadienes leading to 2-pyrrolines. differences in the ligands of the explored rhodium catalysts led to drastic changes in the outcome of the reaction. The optimized conditions for aryl enones (287) involved the use of $\mathrm{Rh}_{2}(\mathrm{~S}-\mathrm{PTV})_{4}$ (4 mol\%), using 2 equivalents of the triazole substrate in dry DCM, at $130{ }^{\circ} \mathrm{C}$ during 1.5 hours, under nitrogen atmosphere.

Tang et al. reported a rhodium(II)-catalyzed formal aza-[3+2] cycloadditions of 1-sulfonyl 1,2,3-triazoles 283 with $(E)$-1-aryl1,3-butadienes 289 leading to 2-pyrrolines 290 (Scheme 100). ${ }^{146}$ The key intermediaries, the $\alpha$-imino rhodium carbenes (azavinyl carbenes), were readily prepared from $\mathrm{N}$-sulfonyl1,2,3-triazoles using $\left[\mathrm{Rh}_{2}(\mathrm{oct})_{4}\right]$ as the rhodium catalyst (in 1,2-DCE at $140{ }^{\circ} \mathrm{C}$ for $12 \mathrm{~h}$ ). The racemic 2-pyrrolines 290 are isolated as the sole products in very good yields (typically 60$95 \%)$. When (Z)-1-aryl-1,3-butadiene instead of the $E$ analog are used, a $[4+3]$ cycloaddition to give 2,5-dihydroazepine products was predominant. With 1,1-diphenyl-, 1-phenyl-2-methyl-, and 1-TBSO-substituted 1,3-dienes as the diene, 2-pyrrolines are the main product (typical yields of $23-90 \%$ ).

Zhang reported the one-pot rhodium-catalyzed intramolecular hydroaminomethylation of substituted cinnamylamines 291 to generate the 4-aryl-2,3-dihydropyrroles 292 with moderate to excellent yields (typically $52-99 \%$, Scheme 101). ${ }^{\mathbf{1 4 7}}$ The reaction is performed with $\mathrm{H}_{2} / \mathrm{CO}$ in a $1 / 1$ ratio at 20 bar pressure. Triphenylphosphine was the best phosphorous ligand for this reaction. Variations in the amine substituents with different $\mathrm{R}^{2}$ alkyl group and electron-withdrawing substituents at the $\mathrm{R}^{1}$ phenyl ring of the cinnamyl group all gave very good yields.

Zhang and co-workers ${ }^{\mathbf{1 4 8}}$ described a rhodium-catalyzed intermolecular [3 +2$]$ cycloaddition of chiral vinylaziridines 294 and alkynes 293 to give optically active 2-pyrrolines 295 (Scheme 102). The optimized conditions used $5 \mathrm{~mol} \%$ of $\left[\mathrm{Rh}(\mathrm{NBD})_{2}\right] \mathrm{BF}_{4}$ in 1,2-dichloroethane (room temperature, 15 $\mathrm{min}$ ). The procedure gives good to excellent yields of product for both internal and terminal alkynes 293 and for several substituted vinylaziridines $\mathbf{2 9 4}$. In addition, a complete transfer

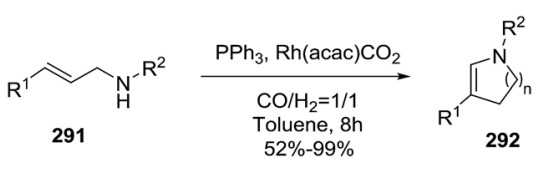

Scheme 101 Rh-catalyzed intramolecular hydroamino-methylation reaction leading to 2 -pyrrolines.

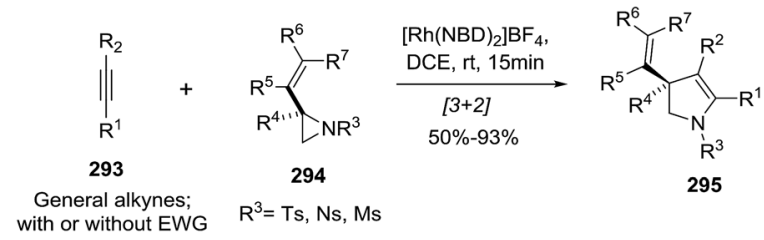

Scheme 102 Rhodium-catalyzed intermolecular [3 + 2] cycloaddition of chiral vinylaziridines and alkynes towards optically active 2pyrrolines. 


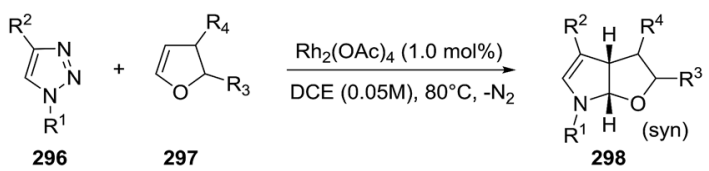

298a, $R^{1}=T s, R^{2}=P h, R^{3}=H, R^{4}=H 88 \%(2.5 h)$

298b, $R^{1}=M s, R^{2}=P h, R^{3}=H, R^{4}=H 70 \%(3 h)$

298c, $\mathrm{R}^{1}=\mathrm{SO}_{2} i-\mathrm{Pr}, \mathrm{R}^{2}=\mathrm{Ph}, \mathrm{R}^{3}=\mathrm{H}, \mathrm{R}^{4}=\mathrm{H} 56 \%(4 \mathrm{~h})$

298d, $R^{1}=\mathrm{SO}_{2}-4-\mathrm{MeOC}_{6} \mathrm{H}_{4}, \mathrm{R}^{2}=\mathrm{Ph}, \mathrm{R}^{3}=\mathrm{H}, \mathrm{R}^{4}=\mathrm{H} 63 \%(4.5 \mathrm{~h})$

298e, $R^{1}=\mathrm{SO}_{2}-4-\mathrm{ClC}_{6} \mathrm{H}_{4}, \mathrm{R}^{2}=\mathrm{Ph}, \mathrm{R}^{3}=\mathrm{H}, \mathrm{R}^{4}=\mathrm{H} 52 \%(1.5 \mathrm{~h})$

298f, $\mathrm{R}^{1}=\mathrm{SO}_{2}-4-\mathrm{CF}_{3} \mathrm{C}_{6} \mathrm{H}_{4}, \mathrm{R}^{2}=\mathrm{Ph}, \mathrm{R}^{3}=\mathrm{H}, \mathrm{R}^{4}=\mathrm{H} 70 \%(1 \mathrm{~h})$

298g, $R^{1}=T s, R^{2}=3-M_{e} C_{6} H_{4}, R^{3}=H, R^{4}=H 68 \%(3 h)$

298h, $R^{1}=T s, R^{2}=4-M_{e} C_{6} H_{4}, R^{3}=H, R^{4}=H 71 \%(2 h)$

298i, $R^{1}=\mathrm{Ts}, \mathrm{R}^{2}=2-\mathrm{MeOC}_{6} \mathrm{H}_{4}, \mathrm{R}^{3}=\mathrm{H}, \mathrm{R}^{4}=\mathrm{H} 65 \%(3 \mathrm{~h})$

298j, $R^{1}=T s, R^{2}=4-M_{e O C} H_{4}, R^{3}=H, R^{4}=H$ 71\%(1 h)

298k, $R^{1}=\mathrm{Ts}, \mathrm{R}^{2}=3-\mathrm{ClC}_{6} \mathrm{H}_{4}, \mathrm{R}^{3}=\mathrm{H}, \mathrm{R}^{4}=\mathrm{H} 60 \%{ }^{(\mathrm{a})}(4.5 \mathrm{~h})$

298I, $R^{1}=\mathrm{Ts}, \mathrm{R}^{2}=4-\mathrm{ClC}_{6} \mathrm{H}_{4}, \mathrm{R}^{3}=\mathrm{H}, \mathrm{R}^{4}=\mathrm{H} 60 \%{ }^{(\mathrm{a})}(3.5 \mathrm{~h})$

$298 \mathrm{~m}, \mathrm{R}^{1}=\mathrm{Ts}, \mathrm{R}^{2}=3-\mathrm{BrC}_{6} \mathrm{H}_{4}, \mathrm{R}^{3}=\mathrm{H}, \mathrm{R}^{4}=\mathrm{H} 59 \%{ }^{(\mathrm{a})}(5 \mathrm{~h})$
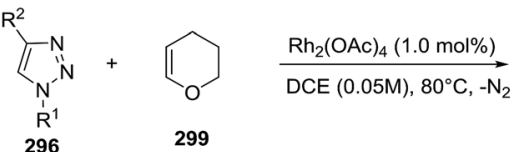

300a, $R^{1}=T s, R^{2}=P h 60 \%(6 h)$

300b, $R^{1}=\mathrm{SO}_{2}-4-\mathrm{MeOC}_{6} \mathrm{H}_{4}, \mathrm{R}^{2}=\mathrm{Ph} 55 \%(5 \mathrm{~h}) \quad 300 \mathrm{e}, \mathrm{R}^{1}=\mathrm{Ts}, \mathrm{R}^{2}=3-\mathrm{CIC}_{6} \mathrm{H}_{4} 50 \%{ }^{(\mathrm{a})}(6 \mathrm{~h})$

(a) $\mathrm{Rh}_{2}(\mathrm{OAc})_{4}(2.0 \mathrm{~mol} \%)$ 298n, $\mathrm{R}^{1}=\mathrm{Ts}, \mathrm{R}^{2}=4-\mathrm{BrC}_{6} \mathrm{H}_{4}, \mathrm{R}^{3}=\mathrm{H}, \mathrm{R}^{4}=\mathrm{H} 55 \%{ }^{(\mathrm{a})}(5 \mathrm{~h})$

298o, $R^{1}=M s, R^{2}=3-M_{e} H_{6} H_{4}, R^{3}=H, R^{4}=H 70 \%(4 h)$

298p, $R^{1}=M s, R^{2}=2-M_{e O C} H_{4}, R^{3}=H, R^{4}=H 70 \%(3 h)$

298q, $R^{1}=M s, R^{2}=4-M_{e O C} H_{4}, R^{3}=H, R^{4}=H 75 \%(2 h)$

298r, $R^{1}=M s, R^{2}=2$-thienyl, $R^{3}=H, R^{4}=H 55 \%(2.5 h)$

298s, $R^{1}=T s, R^{2}=P h, R^{3}=P h, R^{4}=H 89 \%(1.5 h)$

298t, $R^{1}=T s, R^{2}=P h, R^{3}=4-M_{e} C_{6} H_{4}, R^{4}=H 90 \%(4 h)$

298u, $R^{1}=T s, R^{2}=P h, R^{3}=4-C l C_{6} H_{4}, R^{4}=H 91 \%(4 h)$

$298 v, R^{1}=T s, R^{2}=P h, R^{3}=H, R^{4}=P h 84 \%(4 h)$

$298 w, R^{1}=T s, R^{2}=P h, R^{3}=H, R^{4}=4-M_{e O C} \mathrm{H}_{4} 78 \%(3 h)$

298x, $\mathrm{R}^{1}=\mathrm{Ts}, \mathrm{R}^{2}=\mathrm{Ph}, \mathrm{R}^{3}=\mathrm{H}, \mathrm{R}^{4}=4-\mathrm{ClC}_{6} \mathrm{H}_{4} 77 \%(4 \mathrm{~h})$

Scheme 103 Rhodium-catalyzed denitrogenative transannulation of $N$-sulfonyl-1,2,3-triazoles with oxacycloalkenes.

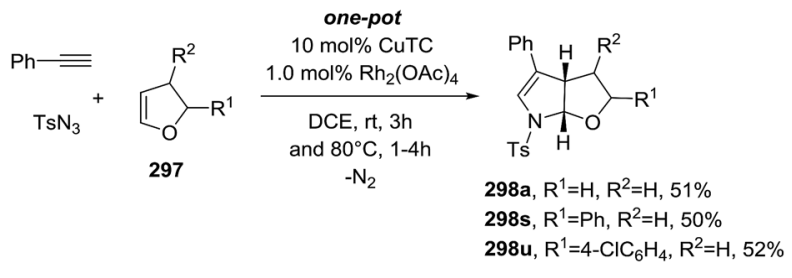

Scheme 104 Rh-catalyzed sequential transannulation.

of chirality from the vinylaziridine to the 2-pyrroline was observed with $90-99 \%$ ee. The use of aliphatic alkynes gave moderate to excellent NMR yields, but the cyclic enamines were unstable to purification since they are hydrolytically labile. With non-symmetric internal alkynes, the product of the reaction (in good yields) is a single regioisomer $\left(\mathrm{R}^{1}=\mathrm{TMS}, \mathrm{R}^{2}=\mathrm{Me}, 64 \%\right.$, $96 \%$ ee; $\mathrm{R}^{1}=4-\mathrm{MeOC}_{6} \mathrm{H}_{4}, \mathrm{R}^{2}=\mathrm{CH}_{2} \mathrm{OMe}, 69 \%$, $96 \%$ ee).

A diastereoselective methodology for the synthesis of tetrahydro-furanodihydropyrroles $\mathbf{2 9 8}$ and tetrahydropyranodihydropyrroles $\mathbf{3 0 0}$ containing $\mathrm{N}, \mathrm{O}$-acetal moieties was proposed by Lee et al. These bicyclic heterocyclic compounds were prepared by a rhodium-catalyzed denitrogenative transannulation of $N$-sulfonyl-1,2,3-triazoles 296 with oxacycloalkenes 297 and 299 (Scheme 103). ${ }^{\mathbf{1 4 9}}$ Optimal conditions for this transformation use $\mathrm{Rh}_{2}(\mathrm{OAc})_{4}(1 \mathrm{~mol} \%)$ as the catalyst, $N$ sulfonyl-1,2,3-triazoles, and oxacycloalkenes in dichloroethane solution $(0.05 \mathrm{M})$ at $80{ }^{\circ} \mathrm{C}$. Under this condition, different $\mathrm{N}$ sulfonyl-1,2,3-triazoles 296 provided the transannulation product without significant variation of yields. The generality of the reaction was proven with the employment of different substituted dihydrofurans 297. Variously substituted racemic 1,2,3-triazoles 296 were obtained as the single diastereomer in moderate to good yield. This protocol was evaluated using 2,3dihydropyran 299. The desired transannulated product was obtained in good yields (50-60\%) (Scheme 103). Finally, the versatility of this rhodium-catalyzed $[3+2]$ cycloaddition was demonstrated by the generation of the 1,2,3-triazoles in situ from terminal alkynes, tosyl azides in presence of 2,3-dihydrofuran and with a CuTC and $\mathrm{Rh}_{2}(\mathrm{OAc})_{4}$ as co-catalysts. This three-component one-pot reaction gave the tetrahydrofuranodihydropyrroles in acceptable yields (typically 50-52\%) and again proves compatibility between the copper and rhodiumcarbenoid catalysts (Scheme 104).

Alcaide and Almendros group reported a rhodium-catalyzed synthesis of 2-pyrrolines $\mathbf{3 0 3}$ from 1-benzenesulfonyl-4-aryl1,2,3-triazoles 302 with allenols 301 (Scheme 105). ${ }^{\mathbf{1 5 0}}$ The protocol makes use of $1 \mathrm{~mol}^{2}$ of $\mathrm{Rh}_{2}(\mathrm{oct})_{4}$ in toluene at reflux to obtain a separable mixture of 2-pyrrolines $\mathbf{3 0 3}$ (only the mayor diastereoisomer is shown) with yields in the range of $31-73 \%$ $\left(\mathrm{R}^{2} \neq \mathrm{H}\right)$. When $\mathrm{R}^{1}$ is aromatic, the diastereoselectivities were modest (d.r. $=55: 45$ to d.r. $=85: 15$ ) but when $\mathrm{R}^{1}$ is aliphatic only the trans diastereomer is observed. However, the yields obtained with $\mathrm{R}^{1}=$ aliphatic are modest (typically $40 \%$ ). The presence of the hydroxyl group in the allene is necessary for the success of the reaction. The mechanism involves the formation of azavinylcarbenes which suffer a nucleophilic addition of the allenol and subsequent azacyclization.

2.2.11. Synthesis of 2-pyrrolines by scandium catalysis. Ghorai et al. ${ }^{151}$ disclosed a practical and enantioselective synthesis of the highly substituted 4,5-dihydropyrroles 306 and/ or 306' via a Domino Ring-Opening Cyclization (DROC) of $\mathrm{N}$ activated aziridines 304 with malononitrile 305 using $\mathrm{Sc}(\mathrm{OTf})_{3^{-}}$ catalysis (Scheme 106). The optimal reaction conditions used $t$ BuOK as a base. Initial screening with monosubstituted $\mathrm{N}$ - 


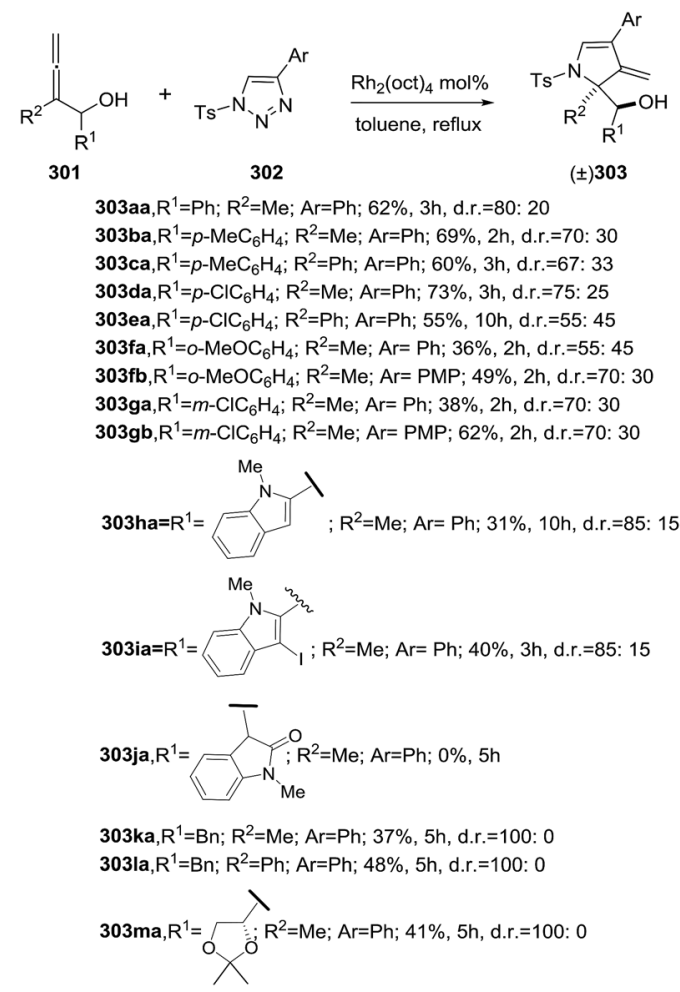

Scheme 105 Rhodium-catalyzed synthesis of 2-pyrrolines 303.

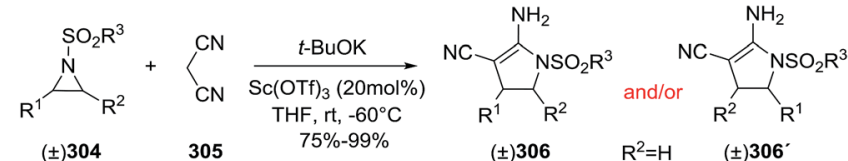

Scheme 106 Synthesis of 2-pyrrolines via an Sc(III)-catalyzed Domino Ring-Opening Cyclization (DROC) of $\mathrm{N}$-activated aziridines with malononitrile.

sulfonyl aziridines 304 gave satisfactory yields of the racemic 4,5-dihydropyrroles (typically $75-99 \%$ ).

The aziridine having the more easily removable $N$-nosyl group gave excellent yields (84-89\%) (Scheme 107).

Enantiomerically pure 4,5-dihydropyrroles 306 were obtained as a single regioisomer from enantiomerically pure alkyl monosubstituted and 2,3-disubstituted aziridines 304. The proposed mechanism involves an $\mathrm{S}_{\mathrm{N}} 2$ nucleophilic attack of the malononitrile anion on the Lewis activated aziridine to generate intermediate $\mathbf{B}$, which undergoes intramolecular cyclization with subsequent protonation and tautomerization to deliver the product (Scheme 108).

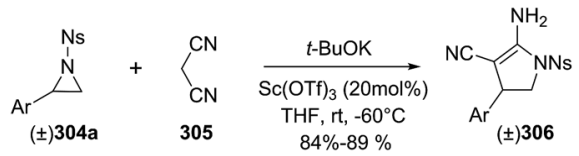

Scheme 107 Domino Ring-Opening Cyclization (DROC) with N-nosyl aziridines.

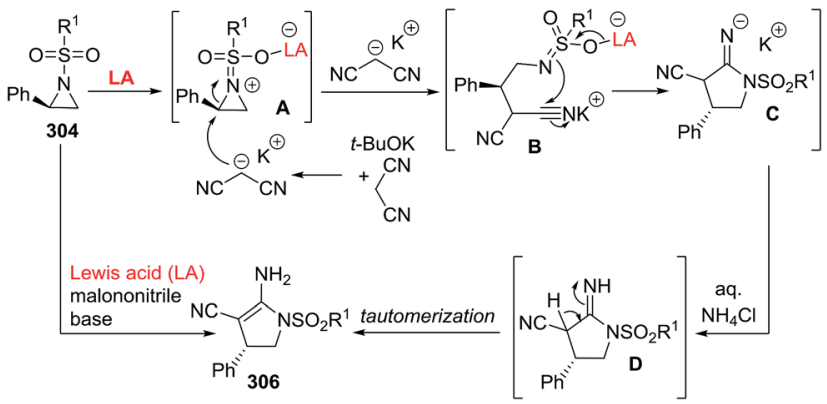

Scheme 108 Proposed mechanism for the Domino Ring-Opening Cyclization (DROC).

In 2015, Xia et al. reported the asymmetric scandiumcatalyzed synthesis of chiral 2,4,5-trisubstituted 2-pyrrolines 309 through the ring-opening cyclization reaction of cyclopropyl ketones 307 with primary amines $308 . .^{152}$ The use of $\mathrm{Sc}(\mathrm{OTf})_{3}$ with the chiral $N, N^{\prime}$-dioxide ligand $\mathbf{L 8}$ gave the best results (Scheme 109). The optimal reaction conditions used the $\mathrm{Sc}(\mathrm{III}) /$ L8 complex (10 $\mathrm{mol} \%$ ) as catalyst and $\mathrm{LiCl}$ as additive (in $\mathrm{CHCl}_{2} \mathrm{CHCl}_{2}$ at $35{ }^{\circ} \mathrm{C}$ for $96 \mathrm{~h}$ ). The methodology tolerates a broad range of cyclopropyl ketones $\mathbf{3 0 7}$ and primary amines 308 with exception of aliphatic amines. In all cases $\left(\mathrm{R}^{3}=\mathrm{Ar}\right)$, the multisubstituted 2-pyrrolines $\mathbf{3 0 9}$ were obtained in good to excellent enantioselectivities (66-96\% ee) and poor to excellent yields $(16-98 \%$ yield). The origin of the enantioselectivity is caused by a kinetic resolution process.

Schneider et al. reported an $\mathrm{Sc}(\mathrm{OTf})_{3}$-catalyzed multicomponent reaction towards multicyclic 2-pyrrolines 315 from 2hydroxy oxime ethers 310, 1,3-dicarbonyls 311, primary amines 312 and indoles 313 or pyrroles 314 (Scheme 110). ${ }^{153}$ This $(2+2$ + 1)-cycloannulation/aza-Friedel-Crafts alkylation sequence provide complex 2-pyrrolines with excellent yields (42-99\%) and diasteroselectivities (d.r. $>95: 5$ ). The procedure tolerates either electron-rich or electron-deficient indoles. However, the use of less nucleophilic $N$-methyl indole resulted in lower yields. Other than that, alkyl substituted pyrroles were sufficiently reactive to form the multicyclic 2-pyrrolines. An intramolecular version of this aza-Friedel-Crafts alkylation was achieved with the amine component tethered to the reactive $\pi$-nucleophile. This is exemplified with the use of 2-(heteroaryl)ethylamine as tryptamine 316. Regarding the scope of 2-hydroxy oxime ethers 310,

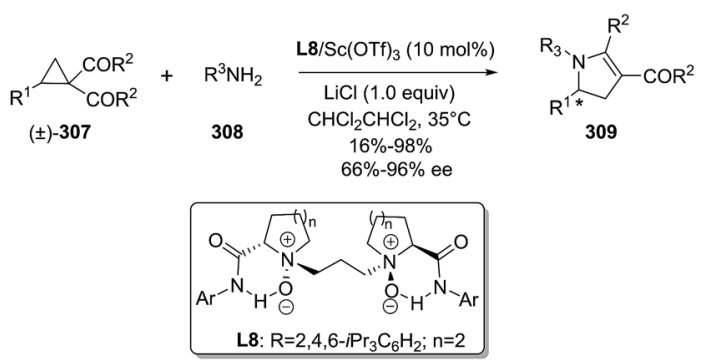

Scheme 109 Asymmetric scandium-catalyzed synthesis of chiral 2,4,5-trisubstituted 2-pyrrolines 309 . 

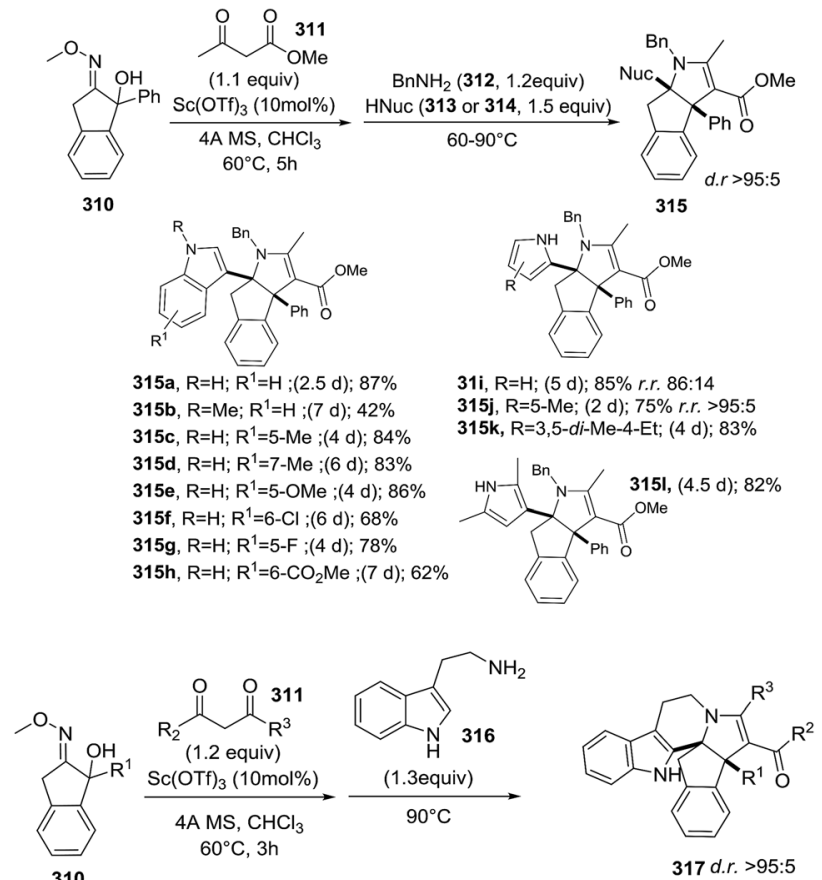

310

317a, $\mathrm{R}^{1}=3-\mathrm{MeC}_{6} \mathrm{H}_{4} ; \mathrm{R}^{2}=\mathrm{OMe} ; \mathrm{R}^{3}=\mathrm{Me} ;>99 \% ; 25 \mathrm{~h} 317 \mathrm{k}, \mathrm{R}^{1}=3-\mathrm{CF}_{3} \mathrm{C}_{6} \mathrm{H}_{4} ; \mathrm{R}^{2}=\mathrm{OMe} ; \mathrm{R}^{3}=\mathrm{Me}$; 317b, $R^{1}=4-M_{e C} \mathrm{H}_{4} ; R^{2}=\mathrm{OMe} ; \mathrm{R}^{3}=\mathrm{Me} ; 97 \% ; 25 \mathrm{~h} \quad 86 \% ; 92 \mathrm{~h}$

317c, $R^{1}=4-{ }^{t} \mathrm{BuC}_{6} \mathrm{H}_{4} ; \mathrm{R}^{2}=\mathrm{OMe} ; \mathrm{R}^{3}=\mathrm{Me} ; 97 \% ; 24 \mathrm{~h} \quad 317 \mathrm{I}, \mathrm{R}^{1}=\mathrm{Me} ; \mathrm{R}^{2}=\mathrm{OMe} ; \mathrm{R}^{3}=\mathrm{Me} ; 75 \% ; 21 \mathrm{~h}$ 317d, $R^{1}=3-M_{e O C} H_{4} ; R^{2}=O M e ; R^{3}=M e ; 94 \% ; 25 h 317 m, R^{1}=B u ; R^{2}=O M e ; R^{3}=M e ; 56 \% ; 45 h$ 317e, $R^{1}=4-M_{e O C} H_{4} ; R^{2}=O M e ; R^{3}=M e ; 80 \% ; 20 h 317 n, R^{1}=P h ; R^{2}=M e ; R^{3}=M e ; 78 \% ; 64 h$ 317f, $R^{1}=3,4-\mathrm{OCH}_{2} \mathrm{OC}_{6} \mathrm{H}_{4} ; \mathrm{R}^{2}=\mathrm{OMe} ; \mathrm{R}^{3}=\mathrm{Me} ; \quad$ 317o, $\mathrm{R}^{1}=\mathrm{Ph} ; \mathrm{R}^{2}=\mathrm{OMe} ; \mathrm{R}^{3}=\mathrm{Et} ; 71 \% ; 122 \mathrm{~h}$ $75 \% ; 43 \mathrm{~h}$ 317p, $R^{1}=\mathrm{Ph} ; \mathrm{R}^{2}=\mathrm{OEt} ; \mathrm{R}^{3}=\mathrm{Me} ; 84 \% ; 24 \mathrm{~h}$ 317g, $\mathrm{R}^{1}=4-\mathrm{PhC}_{6} \mathrm{H}_{4} ; \mathrm{R}^{2}=\mathrm{OMe} ; \mathrm{R}^{3}=\mathrm{Me} ; 60 \% ; 25 \mathrm{~h} \quad 317 \mathrm{q}, \mathrm{R}^{1}=\mathrm{Ph} ; \mathrm{R}^{2}=\mathrm{O} \mathrm{Pr} ; \mathrm{R}^{3}=\mathrm{Me} ; 92 \% ; 23 \mathrm{~h}$ 317h, $R^{1}=2$-naphthyl; $R^{2}=\mathrm{OMe} ; \mathrm{R}^{3}=\mathrm{Me} ; 86 \% ; 32 \mathrm{~h} \quad 317 \mathrm{r}, \mathrm{R}^{1}=\mathrm{Ph} ; \mathrm{R}^{2}=\mathrm{OiBu} ; \mathrm{R}^{3}=\mathrm{Me} ; 85 \% ; 32 \mathrm{~h}$ 317i, $R^{1}=4-\mathrm{ClC}_{6} \mathrm{H}_{4} ; \mathrm{R}^{2}=\mathrm{OMe} ; \mathrm{R}^{3}=\mathrm{Me} ; 98 \% ; 92 \mathrm{~h} \quad 317 \mathrm{~s}, \mathrm{R}^{1}=\mathrm{Ph} ; \mathrm{R}^{2}=$ Oallyl; $\mathrm{R}^{3}=\mathrm{Me} ; 95 \% ; 17 \mathrm{~h}$ 317j, $R^{1}=4-\mathrm{FC}_{6} \mathrm{H}_{4} ; \mathrm{R}^{2}=\mathrm{OMe} ; \mathrm{R}^{3}=\mathrm{Me} ; 89 \% ; 92 \mathrm{~h} \quad 317 \mathrm{t}, \mathrm{R}^{1}=\mathrm{Ph} ; \mathrm{R}^{2}=\mathrm{OBn} ; \mathrm{R}^{3}=\mathrm{Me} ; 92 \% ; 24 \mathrm{~h}$

Scheme $110 \mathrm{Sc}(\mathrm{OTf})_{3}$-catalyzed multicomponent reaction towards multicyclic 2-pyrrolines 317.

the protocol tolerates electron-rich or electron-deficient aryl groups, naphthyl and alkyl groups $\left(\mathrm{R}^{1}\right)$.

2.2.12. Synthesis of 2-pyrrolines by silver. Amat et al. ${ }^{154}$ disclosed the first example of an asymmetric cooperative multicatalytic cascade between isocyanoacetates 318 and $\alpha, \beta$ unsaturated ketones $\mathbf{3 1 9}$ for the rapid construction of enantioenriched 2,3-dihydropyrroles 321, employing the combination of a chiral cinchona alkaloid-derived organocatalyst and silver nitrate as the additive. The scope of the methodology was evaluated with respect to different substituted isocyanoacetates 318 and $\alpha, \beta$-unsaturated ketones 319 which in combination

$$
\begin{aligned}
& 321 \mathrm{a}, \mathrm{R}^{1}=\mathrm{Me}, \mathrm{R}^{2}=\mathrm{H}, \mathrm{R}^{3}=\mathrm{Et}, 49 \%(\mathrm{ee}=74 \%) \\
& 321 \mathrm{~b}, \mathrm{R}^{1}=\mathrm{Me}, \mathrm{R}^{2}=\mathrm{Bn}, \mathrm{R}^{3}=\mathrm{Me}, 85 \%(\mathrm{ee}=16 \%)
\end{aligned}
$$

Scheme 111 Cooperative catalytic synthesis of enantioenriched pyrrolines 321 .

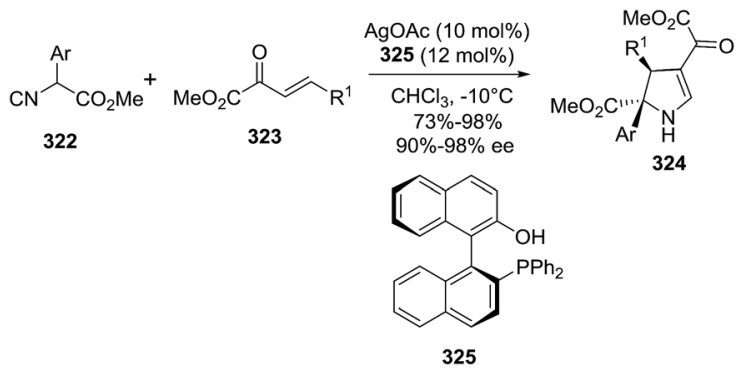

Scheme 112 Asymmetric formal [3 +2$]$ cycloaddition reaction towards chiral 2-pyrrolines.

with cupreine $\mathbf{3 2 0}$ as the chiral base, led to products in moderate enantiomeric excess (Scheme 111).

Simultaneously to the previous report, Gong et al. ${ }^{155}$ published an analogous transformation towards optically active 2pyrrolines 324 but using $\alpha, \beta$-unsaturated esters 323 instead of $\alpha, \beta$-unsaturated ketones as the starting materials (Scheme 112). This study represented the first example of a highly enantioselective $[3+2]$ cycloaddition of isocyanoesters $\mathbf{3 2 2}$ to 2-oxobutenoate esters 323 catalyzed by the silver complex formed from silver acetate and (S)-(2'-hydroxy-1,1'-binaphthyl-2-yl) diphenylphosphine 325. Different substituents present in both reactants were evaluated. In most of the cases 2,3-dihydropyrroles 324 were obtained with excellent yields (73-98\%) and high enantioselectivity (90-98\% ee).

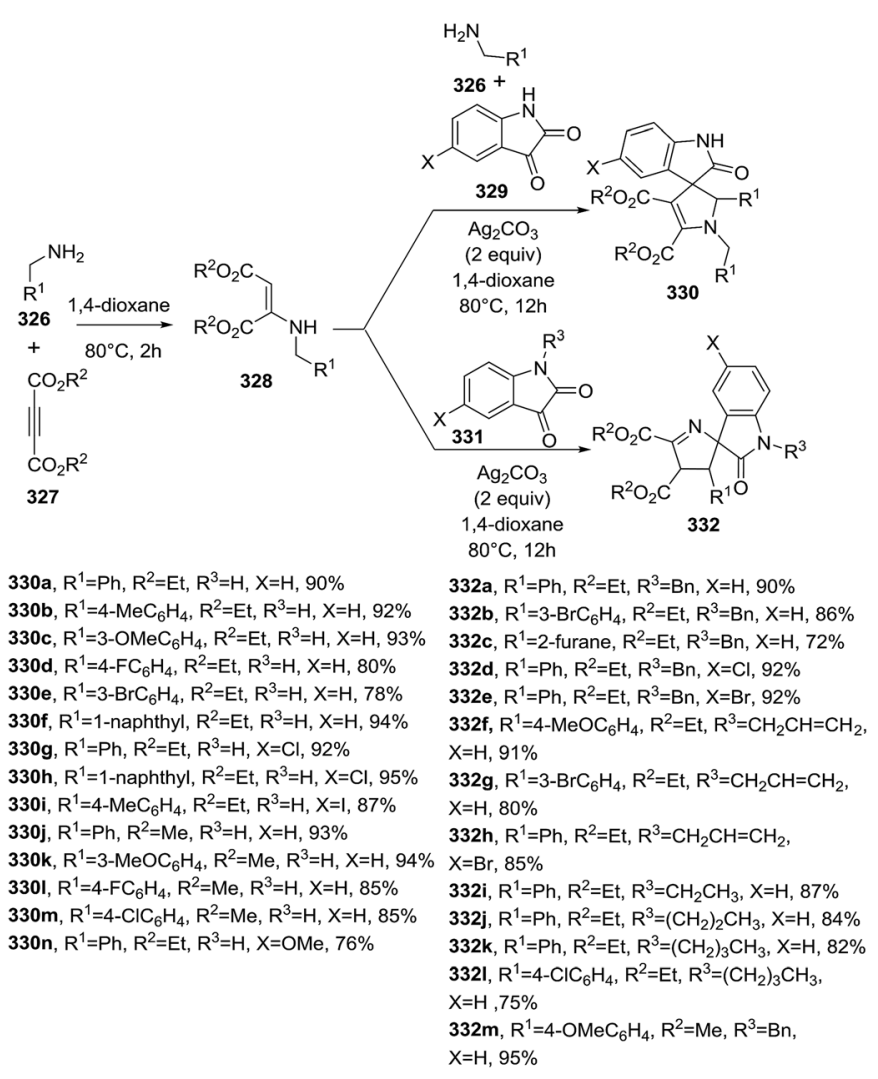

Scheme 113 Regioselective synthesis of spiro-dihydropyrroles 330 and 332 . 
Mukhopadhyay et al. ${ }^{110}$ reported a multicomponent reaction promoted by $\mathrm{Ag}(\mathrm{I})$ through activation of the $\mathrm{C}_{\alpha}\left(\mathrm{sp}^{3}\right)-\mathrm{H}$ bond of the corresponding benzylamine 326 in presence of but-2ynedioate 327 and isatin $329(\mathrm{X}=\mathrm{H})$. A regioselective [1,5]rearrangement gave the spiro-2,3-dihydropyrroles $\mathbf{3 3 0}$ while an alternative [1,3]-rearrangement produced the spiro-1dihydropyrroles 332. The regioselective generation of each heterocyclic core (330 versus 332 ) was achieved under the same reaction conditions involving $\mathrm{Ag}(\mathrm{I})$ and depending on the nature of the group bond to the nitrogen of isatin (Scheme 113). When isatin or $N$-methylisatin is used, a [1,5] rearrangement occurs to afford 2-pyrrolines 330. If the isatin is $N$-substituted with benzyl, allyl, ethyl, propyl, or an $n$-butyl group, a [1,3]-rearrangement occurs to give 1-pyrrolines 332. NMR experiments are consistent with enamine $\mathbf{3 2 8}$ as the common intermediary to both products. The $\mathrm{Ag}(\mathrm{I})$ source of choice was $\mathrm{Ag}_{2} \mathrm{CO}_{3}$, one of the most available and least expensive sources of silver. In addition, the $\mathrm{Ag}_{2} \mathrm{CO}_{3}$ was recycled successfully. The scope of the reaction was evaluated using various derivatives of each starting material $\left(\mathrm{R}^{1}\right.$, $\left.\mathrm{R}^{2}, \mathrm{R}^{3}\right)$. A steric effect of the $N$-substituents is crucial for the fate of the reaction, for instance when $\mathrm{R}^{3}=$ allyl or $n$-butyl produce lower yields compared with $\mathrm{R}^{3}=$ benzyl. When $\mathrm{R}^{3}=$ ethyl or propyl the reaction gives good yields ( $87 \%$ and $84 \%$ respectively).

\subsection{Synthesis of 3-pyrrolines}

Illustrative examples of the importance of the 3-pyrroline ring in natural products and bioactive compounds are displayed in Fig. 5. The 3-pyrroline scaffold is part of the polycyclic core of erysovine (333), a natural erythrinan alkaloid which presents chemotherapeutic properties. ${ }^{9}$ The substituted 2,5-dihydropyrrole is the central ring of the kinesin spindle protein inhibitor 334 (ref. 19) and of compound 335. ${ }^{156}$ Both of these compounds exhibit antitumoral activity. Spirooxindol derivative 336 (ref. 157) has potent anti-microbial activity. The 2,5-dihydropyrrole formyl hydroxyamino derivatives 337 are peptide deformylase inhibitors achieving better in vitro antibacterial activities than existing drugs. ${ }^{158} \mathrm{~A}$ set of synthetic $N$-3-pyrrolines amino arylacetamide derivatives $\mathbf{3 3 8}$ exhibited potent and high selectivity as kappa-agonists with potentially safer analgesic activity. ${ }^{17}$

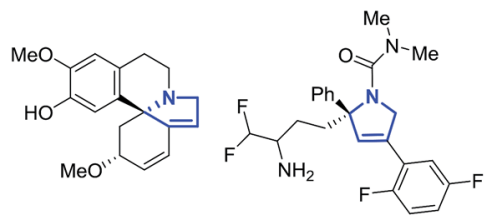

333: Antitumoral

334: Antitumoral

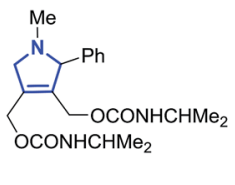

335: Antitumoral

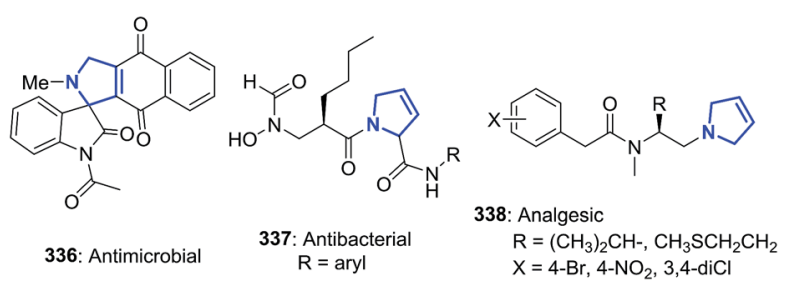

Fig. 5 Selected examples of biologically active 3-pyrrolines.
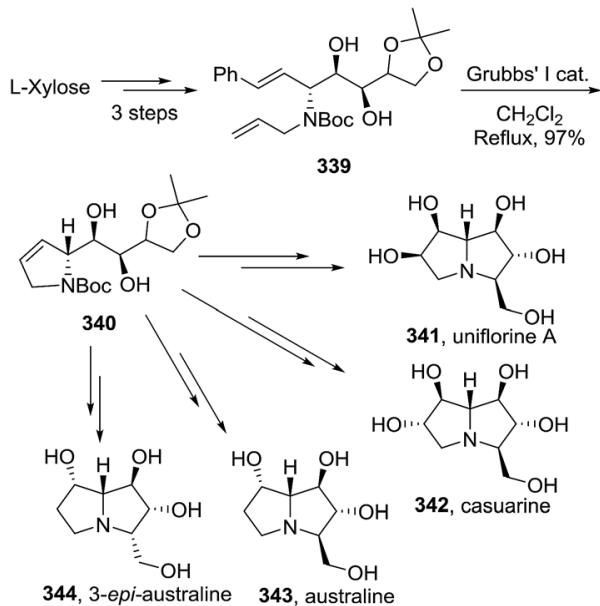

Scheme 114 Synthesis of pyrrolizidine alkaloids through the 3-pyrroline 340 .

Pyne et al. demonstrated the versatility of using 3-pyrrolines as intermediaries in their synthesis of complex alkaloids. The chiral 3-pyrroline $\mathbf{3 4 0}$ was used as an common intermediate towards the synthesis of the glycosidase inhibitors uniflorine A (341), casuarine (342), australine (343), and 3-epi-australine (344). ${ }^{32}$ The chiral pyrroline $\mathbf{3 4 0}$ is available in gram scale starting from L-xylose and using a ring-closing metathesis of 339

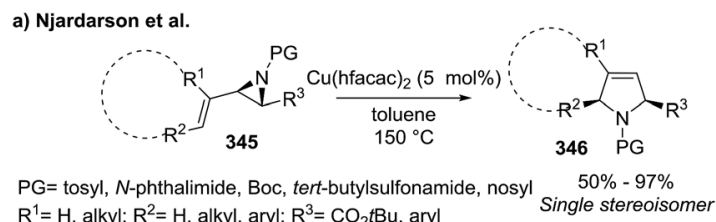
$\mathrm{R}^{1}=\mathrm{H}$, alkyl; $\mathrm{R}^{2}=\mathrm{H}$, alkyl, aryl; $\mathrm{R}^{3}=\mathrm{CO}_{2} \mathrm{tBu}$, ary

b) Zhou et al.

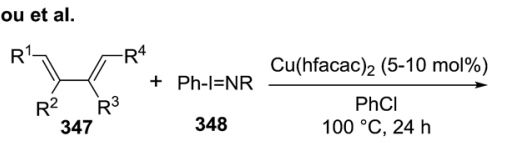

$\mathrm{R}=$ tosyl, nosyl; $\mathrm{R}^{1}, \mathrm{R}^{2}, \mathrm{R}^{3}, \mathrm{R}^{4}=\mathrm{H}$, alkyl, aryl, $\mathrm{CO}_{2} \mathrm{Et}$

Mechanism insights
Vynil aziridene intermediate:

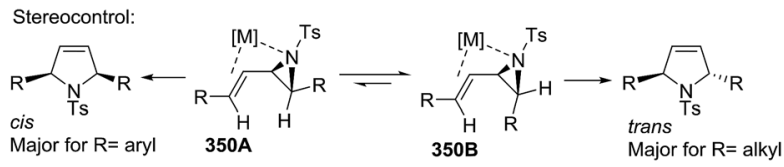

c) Yorimitsu/Oshima et al.
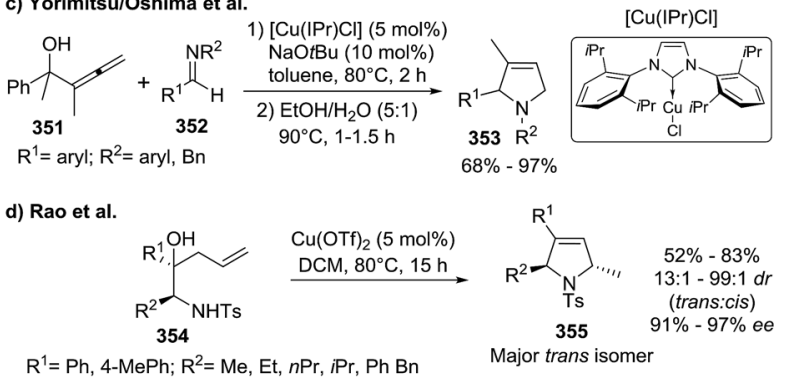

Scheme 115 Different synthetic approaches toward 3-pyrrolines via copper catalysis reported in 2010-2011. 
as a key step. The pyrrolizidine alkaloids 341-344 were obtained in satisfactory overall yields in several steps from 340 (Scheme 114).

2.3.1. Synthesis of 3-pyrrolines by copper catalysis. Between 2010 and 2011, several groups used known (but adapted) synthetic approaches to generate 3-pyrrolines under green, copper-catalyzed conditions. The Njardarson group ${ }^{\mathbf{1 5 9}}$ developed the $\mathrm{Cu}(\text { hfacac })_{2}$ (hfacac: hexafluoroacetylacetonate) catalyzed ring expansion of chiral vinyl aziridines 345 to produce a set of mono and fused 3-pyrroline products 346 (Scheme 115a). This transformation exhibited high stereocontrol, with each starting material produced a single stereoisomer. Using the same catalyst, Zhou and coworkers ${ }^{\mathbf{1 6 0}}$ generated the 3-pyrrolines 349 but via the different mechanism of overall intermolecular [4+1] cycloaddition of 1,3-dienes 347 and the nitrene precursors 348 (Scheme 115b). The more likely mechanism, however, involved [2+1] cycloaddition of a vinylaziridine intermediate (350), followed by ring expansion. This mechanism justifies the stereochemistry of the final pyrroline as determined exclusively by the geometry of the conjugated dienes (as inferred from the proposed metal-chelated vinylaziridines 350A, 350B.). In particular, the 1,4-disubstitued dienes were the best substrates to reach high diastereomeric ratio (cis-product with $\mathrm{R}^{2}$ and $\mathrm{R}^{3}$ substituents pointing towards the same side of the heterocycle compared to the trans-product with those substituents pointing to opposite faces of the ring). In the same year, a report described the synthesis of 3-pyrrolines 353 via tandem allenylation/cyclization reactions catalyzed by the NHC ( $N$-heterocyclic carbene)-ligated copper complex $[\mathrm{Cu}(\mathrm{IPr}) \mathrm{Cl}]$ (Scheme 115c). ${ }^{161}$ The proposed mechanism for this sequence of reactions invokes an amine allenyl intermediate that cyclizes in presence of the copper catalyst. Rao et al. ${ }^{\mathbf{1 6 2}}$ developed a synthetic route directed to trisubstituted 3-pyrrolines 355 via an intramolecular hydroamination of homoallylic amino alcohols 354 mediated by $\mathrm{Cu}(\mathrm{OTf})_{2}$ (Scheme 115d). In this case, the use of enantioenriched aminoalcohols as starting materials produced chiral heterocycles with excellent dr [trans ( $\mathrm{R}^{2}$ and methyl group on the same side of the pyrroline): cis $\left(\mathrm{R}^{2}\right.$ and methyl group on opposite sides of the pyrrolines ring)] and ee values.

Wang and Fang ${ }^{\mathbf{1 6 3}}$ described subsequently the preparation of the highly substituted pyrrolines $\mathbf{3 5 7}$ from the alkynylsubstituted enamides 356 using $\mathrm{Cu}$ (II) catalysis. The key step in this synthesis is the preparation of the linear starting

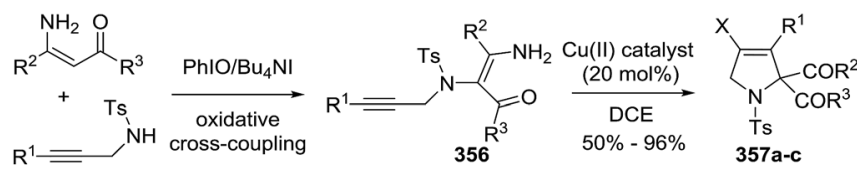

$\mathrm{X}=\mathrm{H}$, Conditions: $\mathrm{Cu}(\mathrm{hfacac})_{2} \cdot \mathrm{H}_{2} \mathrm{O}, 70^{\circ} \mathrm{C}$ $\mathrm{X}=\mathrm{Cl}$, Conditions: $\mathrm{CuCl}_{2}, 25^{\circ} \mathrm{C}$

$\mathrm{X}=\mathrm{Br}$, Conditions: $\mathrm{CuBr}_{2}, 25^{\circ} \mathrm{C}$

$\mathrm{R}^{1}=\mathrm{H}$, alkyl, aryl, naphthyl, 2-thienyl;

$\mathrm{R}^{2}=\mathrm{Me}$, aryl;

$\mathrm{R}^{3}=\mathrm{Me}, \mathrm{OMe}$, aryl

Scheme $116 \mathrm{Cu}($ II)-promoted cyclization of alkynyl-substituted enamides 356 .

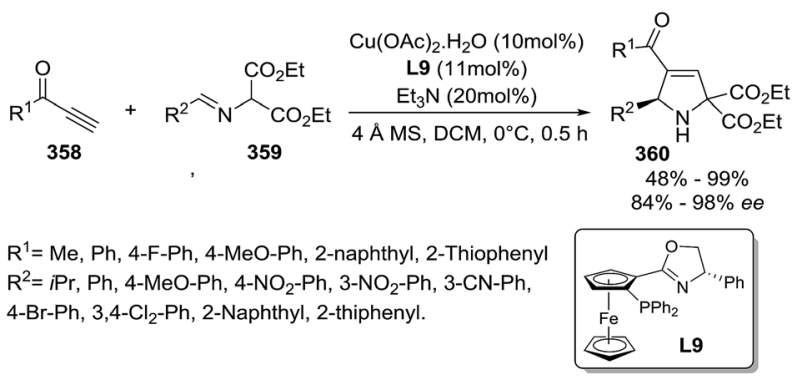

Scheme 117 Asymmetrical 1,3-dipolar cycloaddition of ethynyl ketone 358 to azomethine ylide 359 catalyzed by the complex $\mathrm{Cu}(\mathrm{OAC})_{2} \cdot \mathrm{H}_{2} \mathrm{O} /$ FOXAP derivative $\mathbf{L 9}$.

material 356 via a $\mathrm{PhIO} / \mathrm{Bu}_{4} \mathrm{NI}$-mediated oxidative crosscoupling. Cyclization of such enamides 356 in presence of copper salts led to different halogenated final products $357 \mathbf{a}-\mathbf{c}$ (Scheme 116).

At the same time Tang et al. ${ }^{\mathbf{1 6 4}}$ reported the construction of enantioenriched and highly substituted 3-pyrrolines 360 via asymmetric 1,3-dipolar cycloaddition the ethynyl ketones 358 to the azomethine ylides 359 (Scheme 117). The optimized reaction conditions used $\mathrm{Cu}(\mathrm{OAc})_{2} \cdot \mathrm{H}_{2} \mathrm{O}$ with the FOXAP derivative L9 as the ligand. The pyrrolines were obtained in acceptable to excellent yields (48-99\%) and high enantiomeric excess (84$98 \%)$.

2.3.2. Synthesis of 3-pyrrolines by iron catalysis. Schindler's group described the synthesis of chiral 3-aryl-3-pyrrolines 362 using iron(III)-catalyzed carbonyl-olefin metathesis taking advantages of commercially available amino acids as chiral pool reagents to access metathesis substrates 361 (Scheme 118). ${ }^{\mathbf{1 6 5}}$ The optimized condition makes use of 0.5 equiv. of $\mathrm{FeCl}_{3}$ in dichloroethane at $0{ }^{\circ} \mathrm{C}$ for $1 \mathrm{~h}$ and then warmed to rt. The best results were obtained with carbonyl-olefin metathesis substrates 361 bearing prenyl-derived alkenes and electrondeficient sulfonamide protecting groups (R). This strategy provided 34 aryl-3-pyrrolines in yields up to $99 \%$ with complete stereoretention (up to $98 \%$ ee).

2.3.3. Synthesis of 3-pyrrolines by gold catalysis. Lipshutz and Krause ${ }^{\mathbf{1 6 6}}$ reported in 2011 the first example of 3-pyrroline scaffolds obtained by gold catalysis in micellar systems using PTS or TPGS-750-M as amphiphiles. The heterocycles 364 were efficiently produced from the $\alpha$-functionalized amino allenes 363 via a cycloisomerization promoted by the air-stable aqueous gold(III) solution (Scheme 119a). Interestingly, the reaction presents a broad scope of substrates, and tolerates the TBS ether, ester, and sulfonamide functional groups. More recently

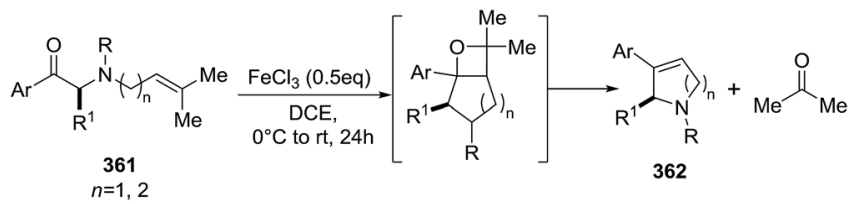

Scheme 118 3-Aryl-3-pyrrolines 362 via catalytic carbonyl-olefin metathesis. 
a) Gold(III) catalysis in aqueous micellar systems

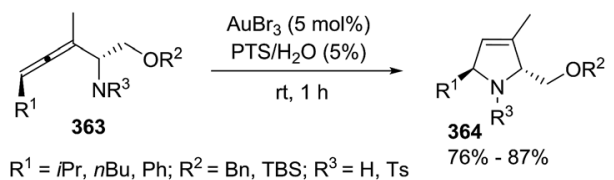

b) Gold(I)-promoted 5-endo-trig cyclization

$$
\mathrm{R}^{1}=\mathrm{Me}, \mathrm{Ph} ; \mathrm{R}^{2}=\mathrm{Ph}, 2 \text {-thienyl, 3-thienyl, TMSE (2-(trimethylsilyl)ethyl) }
$$

Scheme 119 Cyclization of allenyl amines and allenyl tosylamines.

Reissig et al. ${ }^{\mathbf{1 6 7}}$ published an analogous transformation but using $\mathrm{Au}(\mathrm{I})$ catalysis. Starting from allenyl $\mathrm{N}$-tosylamines 365, the 2,3-disubstituted pyrrolines 366 were obtained via 5-endotrig cyclization (Scheme 119b). As previously discussed by the authors in preliminary results, ${ }^{\mathbf{1 6 8}}$ this annulation could be also achieved by either $\operatorname{Ag}(\mathrm{I})$ catalysis or the use of a base such as $\mathrm{KO}^{t} \mathrm{Bu}$. However, the auric catalyst gave higher yields (94-100\%) under mild reaction conditions, and with a more convenient work up (except for the 2-pyrrolyl pyrrolines, which required strongly basic conditions). A similar cycloisomerization was described as part of an interesting multicomponent protocol based on sequential use of metals $(\mathrm{Cu} / \mathrm{Rh} / \mathrm{Au}) .{ }^{169}$ This "three procedures/single work-up" methodology involved a final $\mathrm{Au}(\mathrm{I})$ promoted cyclization of $\alpha$-amino allenes analogous to those described above (see Subsection 2.3.11.: Synthesis of 3-pyrrolines by combined metal catalysis: $\mathrm{Cu} / \mathrm{Rh} / \mathrm{Au}$, Scheme 138).

Several strategies toward the gold-promoted generation of 3pyrroline scaffolds were based on initial gold-alkyne coordination, taking advantage of the high alkynophilicity of this metal. For instance, Shi et al. ${ }^{\mathbf{5 9 , 1 7 0}}$ developed a synthesis of 2,3-disubstituted 3-pyrrolines 368 by the gold(I)-promoted intramolecular cyclization of the functionalized 1,6-diynes 367 (Scheme 120). The use of the $\left(t \mathrm{Bu}_{3} \mathrm{P}\right) \mathrm{AuCl} / \mathrm{AgOTf}$ catalytic system in the presence of water (1 equiv.) was optimal, providing the final cycloadducts in up to $88 \%$ yield. A plausible mechanism for this annulation, implicating two $\mathrm{Au}(\mathrm{I})$ cationic complexes with the two alkynes in 367, was proposed. Even though there is a broad scope of substrates, the terminal alkyne analogues compounds did not provide the pyrroline as a product, but instead the diketone "linear" products were produced.
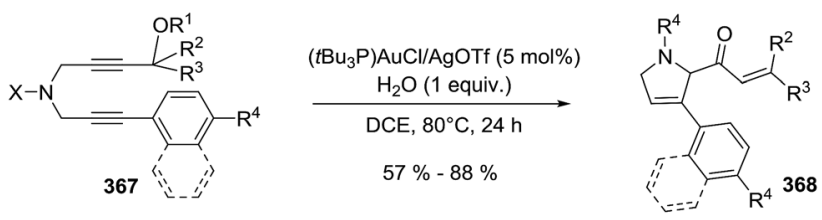

$\mathrm{X}=\mathrm{Bn}, \mathrm{Bs}$ (4-bromobenzenesulfonyl), Ns (4-nitrobenzenesulfonyl), Ts $R^{1}=A c$, Piv; $R^{2}=$ alkyl, Bn; $R^{3}=H, M e ; R^{4}=H, M e, C l$ a)

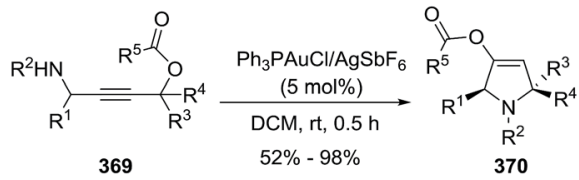

$\mathrm{R}^{1}=\mathrm{H}$, iBu, $\mathrm{Ph}, 4-\mathrm{MeC}_{6} \mathrm{H}_{4}, 4-\mathrm{MeOC}_{6} \mathrm{H}_{4},-\mathrm{CH}_{2} \mathrm{CH}_{2} \mathrm{Br},-\mathrm{CH}_{2} \mathrm{CH}_{2}$ OPiv, $\left(\mathrm{CH}_{2}\right)_{3} \mathrm{OTBDPS}$; $\mathrm{R}^{2} / \mathrm{R}^{3}=\mathrm{H} / \mathrm{H}, \mathrm{H} / 4-\mathrm{MeC}_{6} \mathrm{H}_{4}, \mathrm{H} /$ cyclohexyl, Me/Me; $\mathrm{R}^{4}=\mathrm{Ts}, \mathrm{Bz} ; \mathrm{R}^{5} \mathrm{CO}_{2}=$ OPiv, OAc
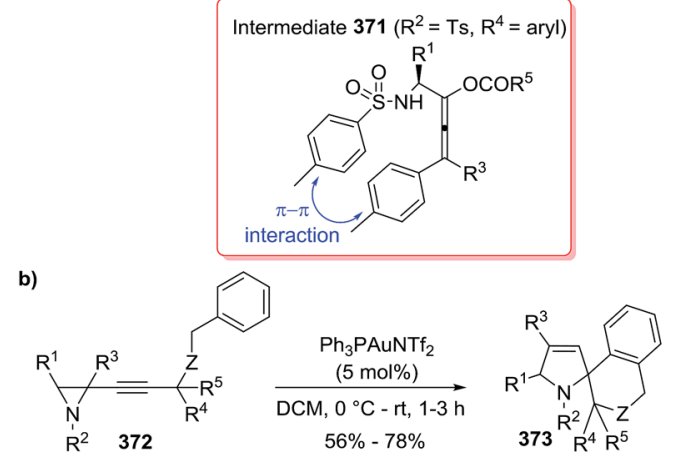

$\mathrm{Z}=\mathrm{O}, \mathrm{CH}_{2}, \mathrm{NTs} ; \mathrm{R}^{1} / \mathrm{R}^{3}=\mathrm{Me} / \mathrm{H}, \mathrm{H} / \mathrm{Ph}, \mathrm{Me} / \mathrm{CH}_{2} \mathrm{OTBS}$, cyclohexyl; $R^{2}=T s, N s ; R^{4} / R^{5}=H / H, M e / M e, P h / P h$, cyclopentyl, H/naphthyl

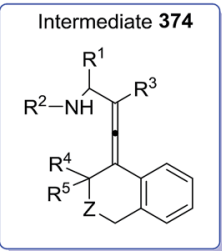

Scheme $121 \mathrm{Au}(\mathrm{I})$-catalyzed cascade reactions involving allenyl intermediates.

Xie, She et al. ${ }^{171}$ applied gold-catalyzed tandem 1,3-acyloxy rearrangement/intramolecular azacyclization to provide the pyrrolines 370 from $\gamma$-amino substituted propargylic esters 369 (Scheme 121a). The authors screened the catalytic system, solvent, and time. The use of $\mathrm{AuPh}_{3} \mathrm{PCl} / \mathrm{AgSbF}_{6}$ in DCM (room temperature, $30 \mathrm{~min}$ ) gave the best results. The proposed mechanism started with gold-alkyne coordination to generate via a 3,3-rearrangement an allenyl intermediate 371, on which an intramolecular $\mathrm{S}_{\mathrm{N}}$ 2-type reaction occurred, followed by a final gold elimination leading to the pyrroline, and regenerating the gold catalytic species. The $\pi-\pi$ interaction present in intermediate 371 explained the cis-stereochemistry (sole products with $\mathrm{R}^{1}$ and $\mathrm{R}^{4}$ on the same side of the pyrroline ring) observed in the 2,5-disubstituted pyrrolines 370 . This cascade of transformations was applied efficiently to a total synthesis of natural alkaloid $( \pm)$-aphanorphine. Similarly, the Blanc and the Pale's groups ${ }^{172}$ developed a novel gold salt methodology to obtain spiro[isochroman-4,2'-pyrrolines] 373 in high yields from aryl-containing alkynylaziridines 372 (Scheme 121b). Similar to the previous example, a cascade reaction through the aminoallenylidene isochromane intermediate $\mathbf{3 7 4}$ leads to the products. In this case, a dual $\sigma$ and $\pi$ gold activation by double gold-coordination to the aziridine and alkynyl moieties, respectively has been proposed. 


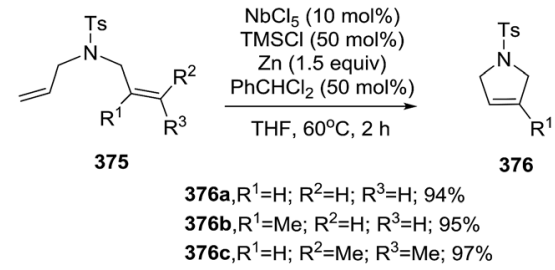

Scheme 122 Synthesis of 3-pyrrolines by in situ-generated niobiumbased catalyst.

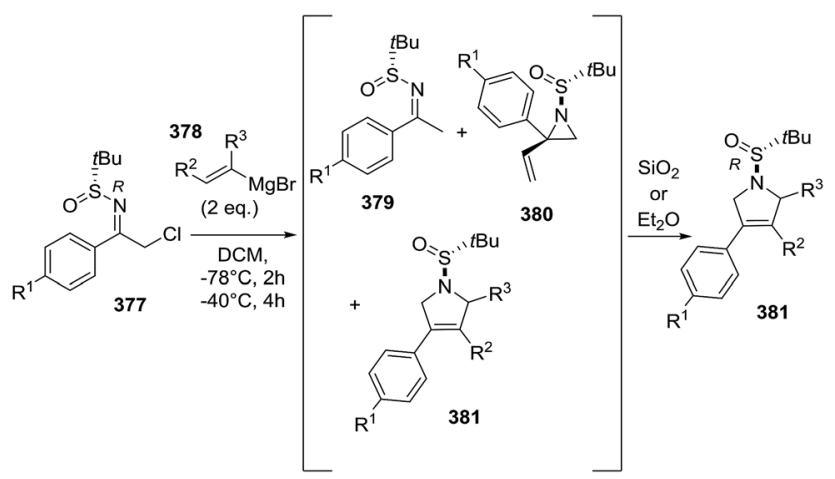

For $(R)-(377), \mathrm{R}^{1}=\mathrm{H}, \mathrm{OMe}, \mathrm{Br}, \mathrm{Cl}, \mathrm{F} ; \mathrm{R}^{2}=\mathrm{R}^{3}=\mathrm{H}$

For $(S)-(377), R^{1}=H ; R^{2}=M e ; R^{3}=H$

Scheme 123 : Synthesis of 3-aryl-3-pyrrolines 381 from vinylmagnesium bromide (378) and chiral $\alpha$-chloro $N$-tert-butanesulfinyl ketimines (377).

2.3.4. Synthesis of 3-pyrrolines by niobium catalyst. Obora et al. developed an active in situ-generated niobium catalyst formed by niobium pentachloride, trimethylsilyl chloride, zinc, benzyl dichloride and THF as solvent. ${ }^{173}$ This Nb-based complex is an active ring-closing metathesis catalyst. 3-Pyrroline derivatives 376 were obtained in quantitative yields starting from $N, N$-diallyl- $p$-toluenesulfonamides 375 when heated at $60{ }^{\circ} \mathrm{C}$ for $2 \mathrm{~h}$ in presence of the Nb-based complex (Scheme 122).

2.3.5. Synthesis of 3-pyrrolines promoted by magnesium. The rearrangement of vinylaziridines to pyrrolines is an exceptionally reliable methodology. The key for its success is the availability of the required highly substituted starting aziridine, and the selection of conditions to control reaction pathway. In this manner, De Kimpe's group ${ }^{\mathbf{1 7 4}}$ developed a mild and efficient addition of vinylmagnesium bromide 378 to chiral $\alpha$ chloro $\mathrm{N}$-tert-butanesulfinyl ketimines (377) to produce in situ the vinylaziridines 380, leading exclusively to the 3-pyrrolines 381 via a $\mathrm{S}_{\mathrm{N}} 2^{\prime}$ ring opening with $\mathrm{C}-\mathrm{N}$ bond cleavage (Scheme 123). The crude reaction mixture contained a combination of dechlorinated imines (378), $N$-sulfinyl-2-aryl-2-vinylaziridenes intermediates (380), and final pyrrolines 381. However, recrystallization from ether or purification by silica chromatography induced the 2-sulfinyl-2-aryl-2-vinylaziridines rearrangement to give the 3-aryl-3-pyrrolines $\mathbf{3 8 1}$ in good yields. Careful analysis gave insight into the effect of the substitution pattern on both the aziridine ring as well as the alkene moiety. In particular, when the $\mathrm{R}^{2}$ substituent was different from hydrogen, the

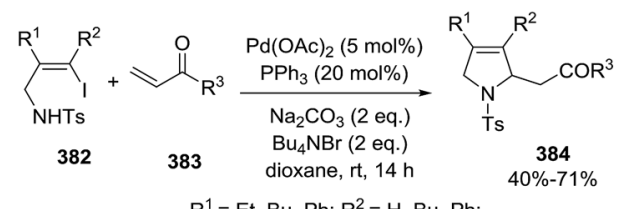

$\mathrm{R}^{3}=\mathrm{Ph}, \mathrm{OMe}$, Ot-Bu, N(OMe)Me, cyclic- $N\left(\mathrm{CH}_{2}\right)_{2}\left(\mathrm{CH}_{2}\right)_{2} \mathrm{O}$

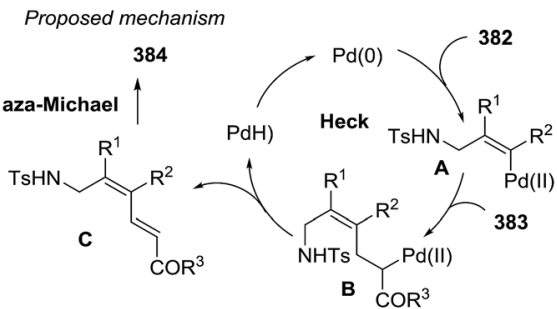

Derivatizations

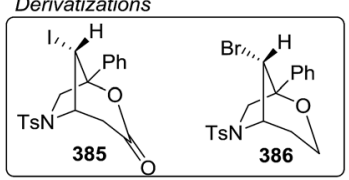

Scheme $124 \mathrm{Pd}(0)$-catalyzed domino Heck-aza-Michael reaction between (Z)-N-(3-iodoallyl)-tosylamides 382 and acrylic esters 383 and related.

pyrroline was not obtained; the corresponding vinylaziridene was produced as the major product instead.

2.3.6. Synthesis of 3-pyrrolines by palladium catalysis. By analysis of the Pd-catalyzed transformations of $(Z)$-vinyl iodides, Tong's group ${ }^{\mathbf{1 7 5}}$ discovered a domino process, combining Heck and aza-Michael reactions, to produce the 3-pyrroline. When tosylamide-substituted vinyl iodides $\mathbf{3 8 2}$ were used in combination with acrylic ester (and related structures 383) in the presence of catalytic $\mathrm{Pd}(\mathrm{OAc})_{2} / \mathrm{PPh}_{3}$ and base (2 equiv.), the domino reaction gave the substituted 3-pyrrolines 384 in 40$71 \%$ yields (Scheme 124). Addition of $\mathrm{Bu}_{4} \mathrm{NBr}$ increased the yield to $65 \%$. Bridged derivatives ( 385 and 386 ) were obtained via synthetic modifications, initializing with ester hydrolysis and ester reduction. The proposed mechanism involves the oxidative addition of vinyl iodide 382 to $\operatorname{Pd}(0)$ to generate $\mathbf{A}$, which undergo a Heck-type reaction with the acrylic ester leading to intermediate $\mathbf{C}$ (Scheme 124). Subsequently, spontaneous aza-Michael cyclization provides the desired $\mathrm{N}$-tosyl pyrrolines.

Another strategy to access 2,5-dihydropyrroles, also based on a Pd-promoted cascade reaction, was developed by Sun et al. ${ }^{\mathbf{1 7 6}}$

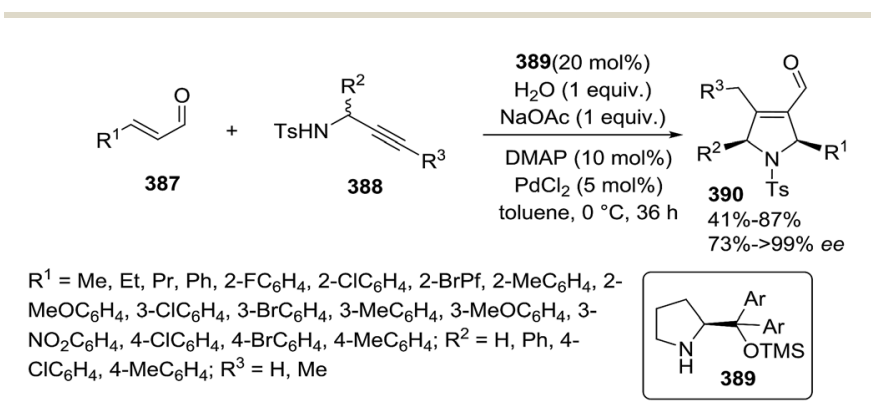

Scheme 125 Asymmetric Pd/amine-catalyzed synthesis of tri- and tetrasubstituted 3-pyrrolines 390 . 


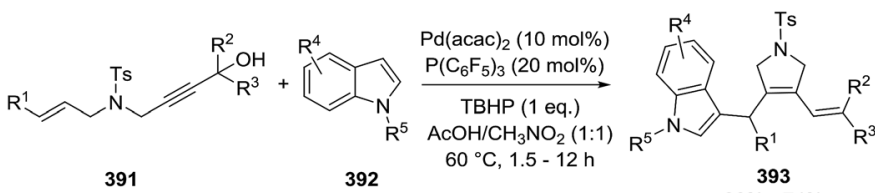

$\mathrm{R}^{1}=\mathrm{H}, \mathrm{Ph} ; \mathrm{R}^{2}=\mathrm{Me}, \mathrm{n}-\mathrm{C}_{6} \mathrm{H}_{13}, \mathrm{Ph}, 4-\mathrm{MeC}_{6} \mathrm{H}_{4}, 4-$ $\mathrm{BrC}_{6} \mathrm{H}_{4}, 4-\mathrm{OMeC}_{6} \mathrm{H}_{4}, \mathrm{~Np}$, furyl; $\mathrm{R}^{3}=\mathrm{H}, \mathrm{Me} ; \mathrm{R}^{4}=$ $\mathrm{H}, 6-\mathrm{Cl}, 7-\mathrm{Me} ; \mathrm{R}^{5}=\mathrm{H}, \mathrm{Me}, \mathrm{Bn}$ TBHP (t-butyl hydroperoxide)

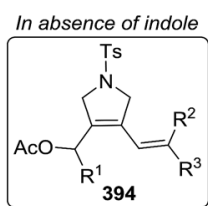

Scheme $126 \mathrm{Pd}$-catalyzed addition of indoles to hydroxyl 1,6-enynes.

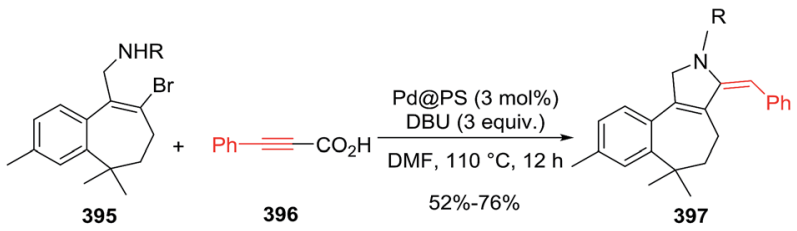

$\mathrm{R}=c-\mathrm{C}_{6} \mathrm{H}_{11}, \mathrm{Bn}, \mathrm{CH}_{2}-3,4,5-$ triOMeC $\mathrm{H}_{4}, \mathrm{CH}_{2}-2-\mathrm{OMeC}_{6} \mathrm{H}_{4}, \mathrm{CH}_{2} \mathrm{CH}_{2} \mathrm{C}_{6} \mathrm{H}_{4}, \mathrm{Ph}, 4-\mathrm{MeC}_{6} \mathrm{H}_{4}$, $\mathrm{Np}$

Scheme 127 Heterogeneous Pd-catalyzed tandem decarboxylative coupling-5-exo cyclization.

In this case the combination of metal and organic catalysis produced chiral 3-pyrrolines 390 from the readily available $\alpha, \beta$ unsaturated aldehydes $\mathbf{3 8 7}$ and $N$-tosyl propargylamines $\mathbf{3 8 8}$ as the starting materials, via an iminium/enamide cascade (Scheme 125). The proposed mechanism integrates the two catalytic cycles. The overall cascade begins with the iminium activation of the aldehyde by the chiral amine $\mathbf{3 8 9}$, followed by an aza-Michael addition and final Pd-promoted carbocyclization, in which the metal coordinates simultaneously the enamide and the alkyne moieties. This cascade organo-metal cooperative catalysis procedure facilitates the production of 3pyrrolines in acceptable to good yields (41-87\%) and with excellent diastereo- (>16:1 (cis : trans) dr, only the major cis diastereomer is shown) and enantioselectivities (73 to >99\% ee). Although limited to the use of terminal alkynes, diverse enantiomerically enriched tri- and tetrasubstituted pyrrolines were produced, presenting a wide scope of substituents at $\mathrm{R}^{1}$ and $\mathrm{R}^{2}$ positions.

The Pd-promoted cycloisomerization of enynes to 3-pyrrolines was exploited by Liang and coworkers (Scheme 126). ${ }^{177}$ The starting $p$-toluensulfonamides 1,6-enynes (391) underwent cycloisomerization in the presence of $\operatorname{Pd}(\mathrm{acac})_{2}$ to generate cyclopropylpalladium complexes, which were captured by the indoles 392 leading to the final 3-pyrrolines derivatives 393 with moderate yields (up to $74 \%$ ). In the absence of the indole, an acetate anion from the reaction medium is incorporated, producing the truncated 3-pyrrolines 394.

The use of polystyrene-supported palladium (Pd@PS) nanoparticles (NPs) has been applied for the generation of fused 3pyrrolines. ${ }^{178}$ This heterogeneous catalyst promotes a domino decarboxylative coupling-cyclization (DCC) from phenylpropiolic acid (396) and aminomethyl benzocycloheptene bromide 395, leading to a new class of bioactive 3-pyrroline

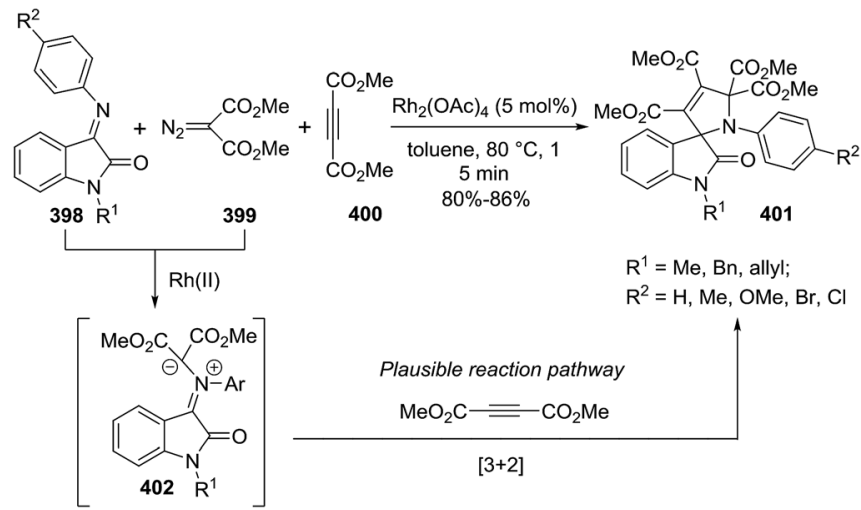

Scheme 128 Multicomponent reactions towards spirooxindolyl pyrroline catalyzed by rhodium.

derivatives 397 with acceptable to good yields (52-76\%) (Scheme 127). The catalytic species was proven to be $\operatorname{Pd}(0)$ in heterogeneous form. Furthermore, the catalyst could be used up to five times without significant decreased activity.

2.3.7. Synthesis of 3-pyrrolines by rhodium catalysis. A Rhcatalyzed multicomponent procedure was developed by Reddy et al. ${ }^{179}$ to obtain highly functionalized spirooxindolyl pyrrolines 401, which can be thought as hybrid drugs, as they present two active moeities (Scheme 128). The optimized multicomponent reaction required cyclic ketimines (398), dimethyl diazomalonate (399), and dimethyl acetylenedicarboxylate (400) in the presence of $\mathrm{Rh}_{2}(\mathrm{OAc})_{2}(5 \mathrm{~mol} \%)$ in benzene $\left(80{ }^{\circ} \mathrm{C}, 15 \mathrm{~min}\right)$. The rhodium is proposed to catalyze the formation of azomethine ylides $\mathbf{4 0 2}$ from 398 and 399, which are subsequently trapped by the alkyne derivatives $\mathbf{4 0 0}$ present in the reaction mixture through Huisgen's cycloaddition, to generate the pyrroline. This methodology results in the remarkably efficient,
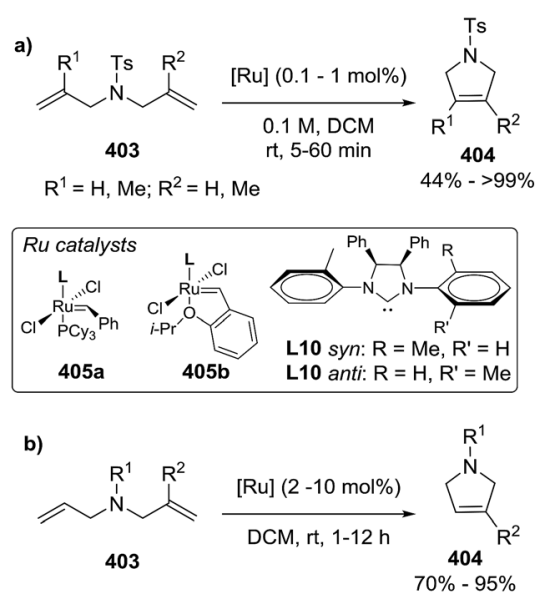

For $\mathrm{R}^{2}=\mathrm{H}: \mathrm{R}^{1}=\mathrm{Cy}, \mathrm{Ph}, 4-\mathrm{FC}_{6} \mathrm{H}_{4}, 4-\mathrm{MeOC}_{6} \mathrm{H}_{4}, \mathrm{Bn}, \mathrm{Ts}$ For $\mathrm{R}^{1}=\mathrm{Ph}: \mathrm{R}^{2}=i-\mathrm{Pr}, n-\mathrm{Pen}, \mathrm{Bn}, \mathrm{Ph}, \mathrm{Np}$

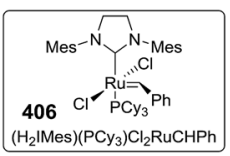

Scheme 129 RCM promoted by Ru catalysts towards 3-pyrrolines. 
single-step production of the complex spirocycles $\mathbf{4 0 1}$ with good to excellent yields (80-86\%).

2.3.8. Synthesis of 3-pyrrolines by ruthenium catalysis. $\mathrm{Ru}-$ catalyzed ring-closing metathesis (RCM) is one of the most efficient and broadly employed synthetic methods for the generation of various cyclic products. ${ }^{180}$ The application of this methodology towards the generation of 3-pyrrolines has been broadly explored, encompassing different aspects such as mechanistic studies with different ruthenium catalysts, ${ }^{\mathbf{1 8 1}}$ an enyne version of the transformation, ${ }^{182}$ and the evaluation of the catalyst loading. ${ }^{183}$ Over the last years, this transformation has been improved and expanded. Most reports describe the $\mathrm{Ru}$ promoted annulation of dialkenyl-substituted amines affording 3-pyrrolines whose substitution patterns were directly determined by the chemical structure achieved during the synthesis of such diolefinic starting materials. Diallyl sulfonamides are commonly used to study this RCM. For example, Perfetto et $a l .{ }^{\mathbf{1 8 1}}$ described the performance of new ruthenium NHC ligand (L10) complexes 405 (Scheme 129a). Kinetics studies showed that the complex catalysts syn-405a and syn405b are remarkably efficient for the RCM of hindered olefins. Samec et al. ${ }^{184}$ expanded the substrate scope, as described by the synthesis of $N$-aryl, $N$-tosyl, and $N$-alkyl pyrrolines from diallylated amines 403, derived from the Pd-catalyzed reaction between allylic alcohols and the corresponding amines (Scheme $\left.129 \mathrm{~b}, \mathrm{R}^{2}=\mathrm{H}\right)$. The linear amine substrates 403 were transformed into the heterocycles $\mathbf{4 0 4}$ in good to excellent yields by RCM using $\left(\mathrm{H}_{2} \mathrm{IMes}\right)\left(\mathrm{PCy}_{3}\right) \mathrm{Cl}_{2} \mathrm{RuCHPh}$ (406). The sequence of reactions exhibited high atom economy, with only water and ethane as side products. A one-pot version of the transformation, with the co-presence of the Pd and Ru metals, was successful. Some years later, Samec's group ${ }^{\mathbf{1 8 5}}$ expanded the scope of this strategy towards unsymmetrical diallylated aromatic amines, which enabled RCM promoted by the same $\mathrm{Ru}$ catalyst to give 3-substituted 3-pyrrolines (Scheme $129 \mathrm{~b}, \mathrm{R}^{2}$ $\neq \mathrm{H})$. The dialkenyl containing amines were obtained by a twostep coupling sequence between aniline and the two corresponding allylic alcohols in presence of in situ-generated Pd $\left[\mathrm{P}(\mathrm{OPh})_{3}\right]_{3}$. An expansion of this methodology was achieved by adding $\mathrm{Fe}\left(\mathrm{III}\right.$ ) (as $\mathrm{FeCl}_{3} \cdot 6 \mathrm{H}_{2} \mathrm{O}$ ) during the cyclization step, leading via a two-step, one-pot procedure to the product of pyrroline oxidation, the pyrrole. In addition, Clayden et al. ${ }^{\mathbf{1 8 6}}$ explored the incorporation of urea functionality at $\mathrm{R}^{1}$ position. Using $N, N$-diallylureas as suitable substrates for the RCM in presence of $5 \mathrm{~mol} \%$ of Grubbs 1st generation catalyst, $N$ [(alkylamino)carbonyl]-2,5-dihydropyrroles were obtained.

Asymmetric syntheses of 3-pyrrolines via RCM promoted by ruthenium, in which the pyrroline stereochemistry is defined by the RCM substrates are also possible. For instance, Hong's and Wang's groups ${ }^{\mathbf{1 8 7}}$ collaborated to explore the used of Zhan 1-B $\mathrm{Ru}$ catalyst to obtain the 2-substituted 3-pyrroline 409 as a single enantiomer (83-87\% ee) from chiral $N$-diallyl $p$-toluensulfonamides $\mathbf{4 1 0}$, whose stereocenter was defined by the quinidine present in the previous reaction between 407 and 408 (Scheme 130a). Similarly, but with a different stereoselective approach, Kamimura's group ${ }^{\mathbf{1 8 8}}$ reported the preparation of optically active 2,5-dihydropyrroles (412) from enantiomerically-enriched aza-Baylis-Hillman adducts (411, Scheme 130b). These key substrates $\mathbf{4 1 1}$ were generated via an optimized domino-reaction procedure from chiral sulfinimines and tert-butyl acrylate. The annulation step promoted by Grubbs' second generation catalyst proceeded smoothly to provide the desired pyrrolines 412 in high yields (92-100\%) and conserving the initial enantiomeric excess (90-98\% ee). In addition, the formal synthesis of the natural pyrrolizidine alkaloid (-)-trachelanthamidine $\mathbf{4 1 4}$ was proposed based on this methodology, to afford the immediate precursor 413. ${ }^{189}$ Another interesting application of chiral pyrrolines as key intermediates in the synthesis of natural products was achieved by Davies and coworkers. ${ }^{190}$ This group developed

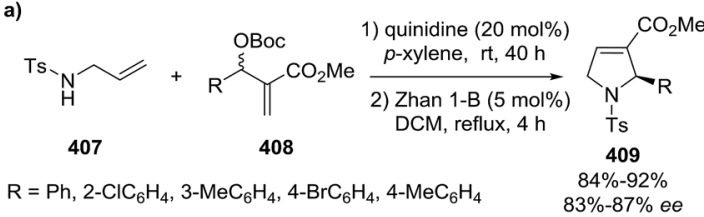

b)

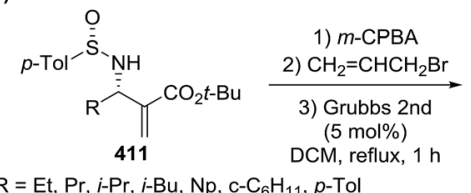

c)

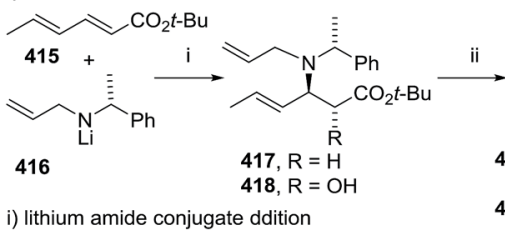

i) lithium amide conjugate ddition

ii) Grubbs I (5 mol\%),DCM, rt, $12 \mathrm{~h}$
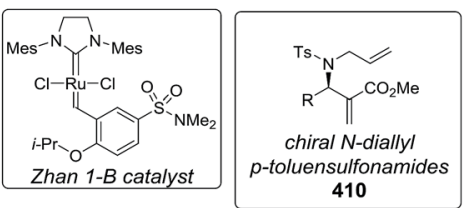

$83 \%-87 \%$ ee

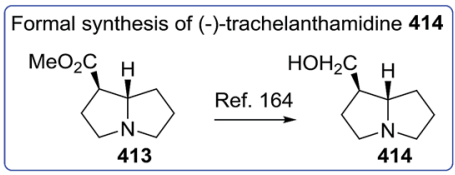

$92 \%-100 \%$

90\%-98\% ee
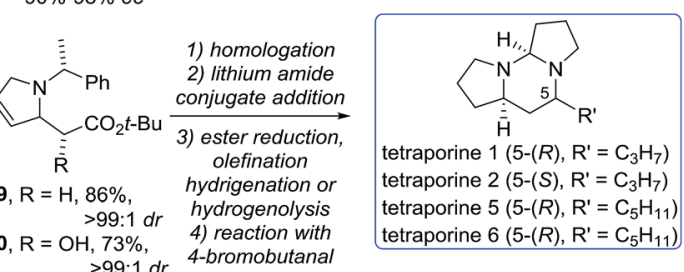

Scheme 130 Asymmetric synthesis of 3-pyrrolines and their applications for the generation of natural products. 


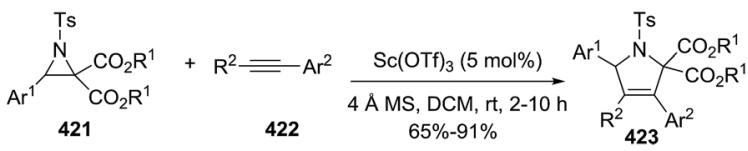

$\mathrm{R}^{1}=\mathrm{Me}$, Et, $i-\mathrm{Pr} ; \mathrm{R}^{2}=\mathrm{Me}, n-\mathrm{Bu}$, cycle- $\mathrm{Pr}, \mathrm{CH}_{2} \mathrm{OMe}, \mathrm{CO}_{2} \mathrm{Me}, \mathrm{Ph}, \mathrm{Br}, \mathrm{TMS}$; $\mathrm{Ar}^{1}=\mathrm{Ph}, 4-\mathrm{FC}_{6} \mathrm{H}_{4}, 4-\mathrm{Cl}-\mathrm{Ph}, 4-\mathrm{BrC}_{6} \mathrm{H}_{4}, 4-\mathrm{NCC}_{6} \mathrm{H}_{4}, 4-\mathrm{NO}_{2} \mathrm{C}_{6} \mathrm{H}_{4}, 4-\mathrm{MeC}_{6} \mathrm{H}_{4}, 4-i-$ $\mathrm{PrC}_{6} \mathrm{H}_{4}, 3-\mathrm{MeC}_{6} \mathrm{H}_{4}, 2-\mathrm{BrC}_{6} \mathrm{H}_{4}$; $\mathrm{Ar}^{2}=4-\mathrm{OMeC}_{6} \mathrm{H}_{4}, 4-\mathrm{MeC}_{6} \mathrm{H}_{4}$

Scheme 131 Sc(III)-catalyzed synthesis of highly substituted 3-pyrrolines from $N$-tosylaziridines and alkynes.

a diastereoselective lithium amide conjugation methodology that enabled the generation of enantiomerically pure $\beta$-amino esters 417 and 418, from lithium $(R)-N$-allyl- $N$-( $\alpha$-methylbenzyl)amides 416 and dienyl ester 415 (Scheme 130c). Subsequent ring closing metathesis of $\mathbf{4 1 7}$ and $\mathbf{4 1 8}$ promoted by Grubbs I catalyst provided the dihydropyrroles as the single diastereoisomers 419 and 420 (>99: $1 \mathrm{dr}$ ). This lithium amide conjugate addition was applied as key stereodefining step during the synthesis of the natural alkaloids (+)-tetraponerine-1, -2, -5, and -6 (Scheme 130c)..$^{37}$ The dehydroxy derivative 319 was used for access to the tricyclic core of the target molecules, which were isolated enantiomerically pure and in acceptable overall yields from commercial starting materials.

2.3.9. Synthesis of 3-pyrrolines by scandium catalysis. As previously mentioned, aziridines are convenient substrates for the generation of pyrrolines scaffolds. For instance, these ringstrained three-membered cyclic amines (421) produce azomethine ylides via selective $\mathrm{C}-\mathrm{C}$ heterolysis under the mild reaction conditions involving $\mathrm{Sc}(\mathrm{OTf})_{3}$ as the Lewis acid (Scheme 131). ${ }^{191}$ These in situ generated dipoles underwent 1,3-dipolar cycloadditions with various electron-rich alkynes (422) to afford the highly substituted 3-pyrrolines 423. This scandiumcatalyzed $[3+2]$ cycloaddition afforded the desired final

a) Classic Ag-catalyzed 5-endo-trig cyclization with final protodemetallation step

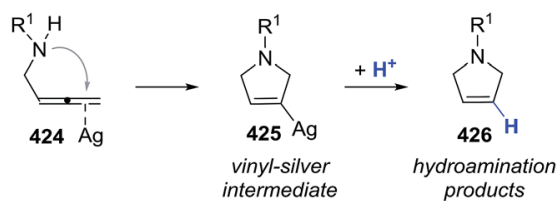

$\mathrm{R}^{1}=\mathrm{H}$, alkyl, $\mathrm{Ph}, \mathrm{Ts}, \mathrm{Bn}$, acyl

b) Ag-catalyzed 5-endo-trig cyclization with final chlloroamination step

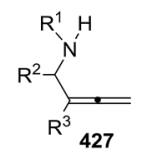

$\mathrm{R}^{1}=\mathrm{Ph}, \mathrm{PMB}$

$\mathrm{R}^{2}=\mathrm{Ph}, 4-\mathrm{BuCOC}_{6} \mathrm{H}_{4}, 4-\mathrm{MeO}_{2} \mathrm{CC}_{6} \mathrm{H}_{4}, 4-\mathrm{CNC}_{6} \mathrm{H}_{4}, 4-\mathrm{BrC}_{6} \mathrm{H}_{4}, 4-\mathrm{IC}_{6} \mathrm{H}_{4}, 4-\mathrm{TBSOC}_{6} \mathrm{H}_{4}$ $\mathrm{R}^{3}=\mathrm{Me}, \mathrm{Ph}$

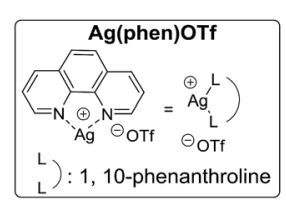

Scheme 132 Cyclization of allenic amines catalyzed by Ag(।). heterocycles as a single regioisomer in good yields (65-91\%) and with a diverse substitution pattern. Further synthetic modifications of the obtained 3-pyrroline core diversified the substitution, and allowed the transformation of these heterocycles into pyrroles, 1-pyrrolines, and pyrrolidines.

2.3.10. Synthesis of 3-pyrrolines by silver catalysis. The use of catalytic silver to produce 3-pyrrolines from aminecontaining allenes has been broadly explored. ${ }^{45,168,192,193}$ Usually in these cases, diversely $N$-protected allenic amines $\mathbf{4 2 4}$ underwent $\mathrm{AgNO}_{3}$-catalyzed 5-endo-trig cyclization towards the 3-pyrrolines, involving a protodeargentation of the vinyl-silver intermediate 425 as the final step (hydroamination products 426, Scheme 132a). A novel modification of this approach incorporated different electrophiles, instead of the proton. ${ }^{194}$ This concept was probed successfully using $N$-chlorosuccinimide (NCS) as the electrophilic species to obtain the halogenated 3-pyrrolines 428 from allene 427 (Scheme 132b). The annulation was achieved with catalytic [Ag(phe)OTf], as a silver catyalyst with increased air and moisture stability, and lutidine as the base. The cyclic products of this chloroamination were isolated in up to $94 \%$ yield. The proposed mechanism is analogous to the previously indicated mechanism. Ag-allene coordination (A) leads to the cationic silver complex $\mathbf{B}$, followed by nitrogen intramolecular attack with corresponding cyclization (C), but with final chlorination by NCS instead of a protodemetallation (Scheme 133). The presence of base is essential, as the base neutralizes the charged species $\mathbf{C}$ and prevents protonation as a side reaction. Unfortunately, all attempts to use $\mathrm{N}$-bromosuccinimide (NBS) failed to give bromodihydropyrroles. Nonetheless, the chloropyrrolines were able to provide different pyrrolines by substitution of chloride, as well as the corresponding chloro-pyrrole cores after oxidative conditions.

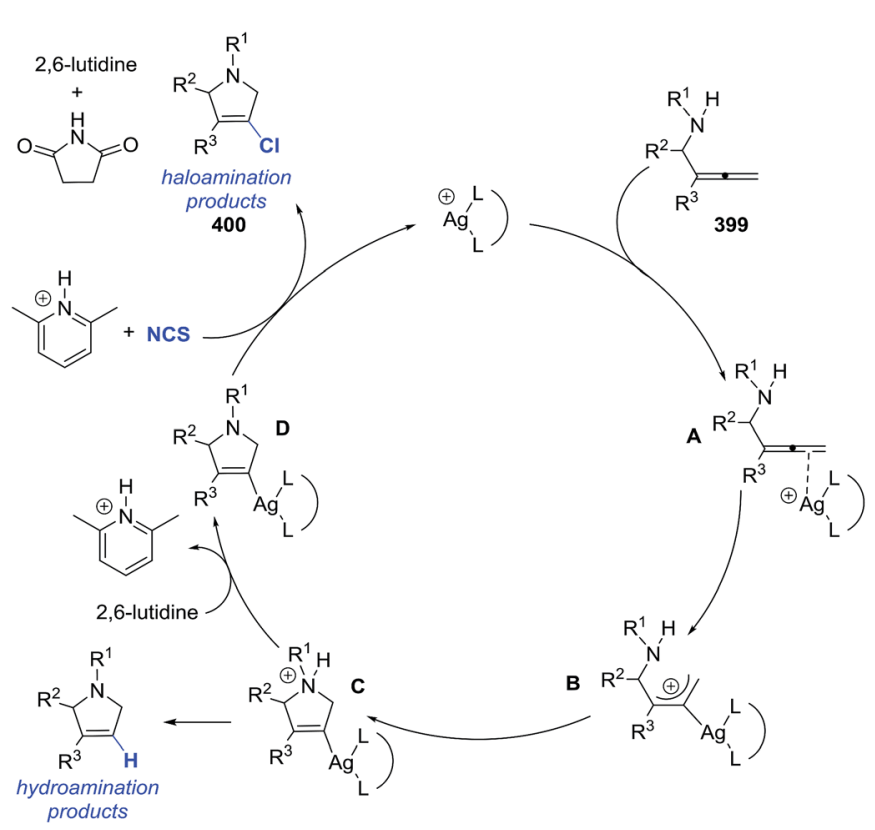

Scheme 133 Proposed mechanism for the formation of chloro-3pyrrolines 428. 


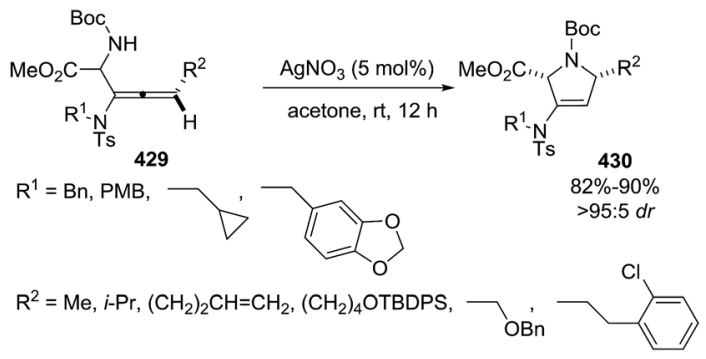

Scheme $134 \mathrm{AgNO}_{3}$-catalyzed synthesis of 2,5-disubstituted 3-pyrrolines 430 .

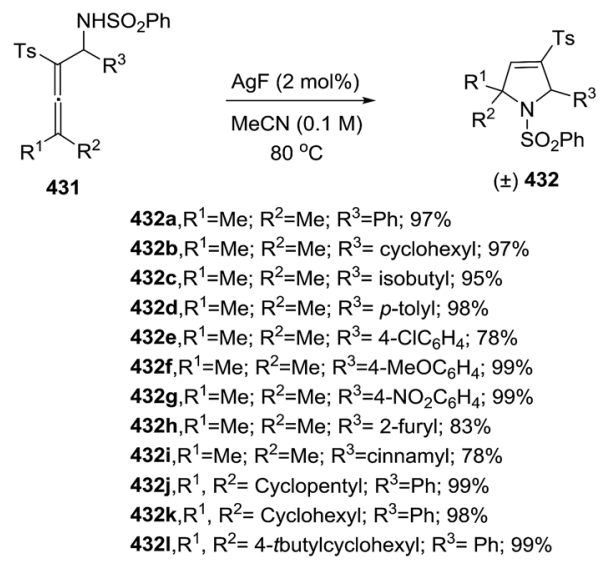

Scheme 135 Synthesis of 3-sulfonyl-2,5-disubstituted-3-pyrrolines 432 catalyzed by silver fluoride.

Based on the same strategy, the use of $\mathrm{N}$-sulfonamidesubstituted allenylamines 429 enabled the preparation of 2,5disubstituted 3-pyrrolines $\mathbf{4 3 0}$ (Scheme 134). ${ }^{195}$ This cyclization proceeded smoothly with $\mathrm{AgNO}_{3}$ to afford the heterocycles in excellent yields (82-90\%) and with high diastereoselectivity (>95 : $5 \mathrm{dr}$, only the major cis-diastereomer is shown).

Harmata et al. treated a series of allenyl sulfonamides $\mathbf{4 3 1}$ with $2 \mathrm{~mol} \%$ of $\mathrm{AgF}$ in acetonitrile at reflux to obtain the corresponding 3-sulfonyl-2,5-disubstituted-3-pyrrolines 432 with yields ranging from 78 to $99 \%$ (Scheme 135). ${ }^{196}$ The 5-endo-trig cyclization catalyzed by silver tolerates phenyl rings with electron-withdrawing and electron-donating groups, heteroaromatic moieties and aliphatic chains in position $\mathrm{R}^{3}$. Cyclic system like cyclopentyl and cyclohexyl groups in position $\mathrm{R}^{1}$ and $\mathrm{R}^{2}$ are rapidly transform in product indicating that there are no steric effects.

Wang et $a .^{197}$ reported a novel generation of 2,5-dihydropyrroles 434 from ketopropargylamines 433 via a 5-endo-dig cyclization promoted by $\mathrm{Ag}(\mathrm{I})$ through a Conia-ene-type reaction (Scheme 136). This report represents the first example of transition metal-catalysis of Conia-ene reactions employing monocarbonyl groups as starting materials. The reaction was compatible with a broad scope of functional groups, and with both terminal and internal alkynes. The reaction proceeded efficiently with tosyl and nosyl groups in $\mathrm{R}^{2}$ position. However, the reaction failed if the $N$-substituent was alkyl, aryl, or acyl.

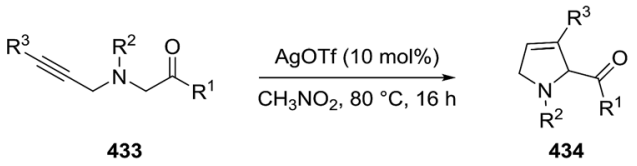

$\mathrm{R}^{1}=\mathrm{Et}$, Ph, furyl, naphtyl, 2- $\mathrm{MeOC}_{6} \mathrm{H}_{4}, 2-\mathrm{BrC}_{6} \mathrm{H}_{4}, 2-\mathrm{FC}_{6} \mathrm{H}_{4}, 3-\mathrm{BrC}_{6} \mathrm{H}_{4}$, 3- $\mathrm{IC}_{6} \mathrm{H}_{4}, 4-\mathrm{MeC}_{6} \mathrm{H}_{4}, 4-\mathrm{FC}_{6} \mathrm{H}_{4}, 4-\mathrm{BrC}_{6} \mathrm{H}_{4}, 4-\mathrm{NO}_{2} \mathrm{C}_{6} \mathrm{H}_{4}, 3,4-$ diClC $_{6} \mathrm{H}_{3}$; $\mathrm{R}^{2}=\mathrm{Ts}, \mathrm{Ns} ; \mathrm{R}^{3}=\mathrm{H}, \mathrm{Me}, \mathrm{Ph}$

Scheme 136 Silver(ı)-catalyzed Conia-ene type reaction.

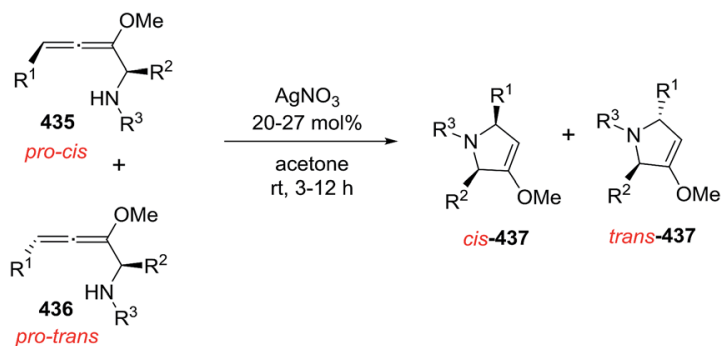

Scheme 137 Silver-catalyzed cyclization to give polysubstituted 3pyrrolines 437.

The final pyrrolines were obtained in acceptable to excellent yields (49-94\%). Some of the pyrrolines were oxidized successfully to the corresponding pyrroles.

Reissig et al. published the synthesis of 1,2,3,5-tetrasubstituted 3-pyrrolines $\mathbf{4 3 7}$ from a mixture of pro-cis and pro-trans allenylamines (435 and 436) using silver nitrate as catalyst at room temperature (Scheme 137). ${ }^{\mathbf{1 9 8}}$ The cis and trans 3-pyrrolines (cis-437 and trans-437) were obtained in good yields. However, the silver-catalyzed cyclization was not stereoespecific since silver nitrate would allow a configurational isomerization of axially chiral allenylamines 435 and 436 .

2.3.11. Synthesis of 3-pyrrolines by combined metal catalysis: $\mathbf{C u} / \mathbf{R h} / \mathbf{A u}$. Miura, Murakami and coworkers ${ }^{169}$ reported a one-pot, multistep-pathway to 3-pyrrolines $\mathbf{4 4 1}$ from terminal alkynes 438, sulfonyl azides 439, and propargyl alcohols 440. The relay action of a set of three metals (copper, rhodium and gold: Scheme 138a) achieved three sequential reactions culminating with a single final work-up and purification. The plausible mechanism uses initial $\mathrm{Cu}(\mathrm{I})$-catalyzed 1,3-dipolar cycloaddition between alkyne $\mathbf{4 3 8}$ and azide $\mathbf{4 3 9}$ to provide the triazole 442a, which equilibrates with the $\alpha$-diazo imine $\mathbf{4 4 2 a ^ { \prime }}$ (Scheme $138 b$ ). Intermediate $\mathbf{4 4 2 a ^ { \prime }}$ reacts with $\mathrm{Rh}$ (II) leading to the $\alpha$-imine rhodium carbene complex $\mathbf{A}$, which reacts with the propargyl alcohol $\mathbf{4 4 0}$ to generate the zwitterionic intermediate B. Rhodium release and a proton shift give the $(Z)$-isomer of $\mathbf{C}$, which at $100{ }^{\circ} \mathrm{C}$ undergoes a spontaneous Claisen-type rearrangement to give the $\alpha$-allenyl- $\alpha$-aminoketone 443 . The final step is a gold-catalyzed cycloisomerization to the 3-pyrroline 441, in a similar manner to the examples described previously in the gold subsection (see Subsection 2.3.3.: Synthesis of 3pyrrolines by gold catalysis, Scheme 119). In addition, the application of sequential independent second Rh-catalyzed and third Au-catalyzed procedures (each step with its adequate work-up) was evaluated, using the corresponding triazoles $\mathbf{4 4 2}$ 
a)

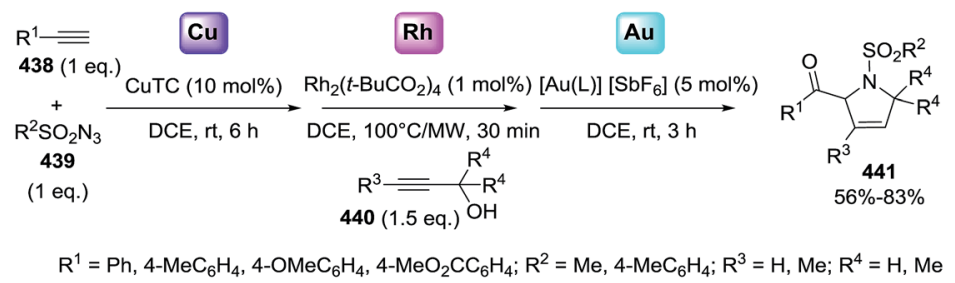

b)

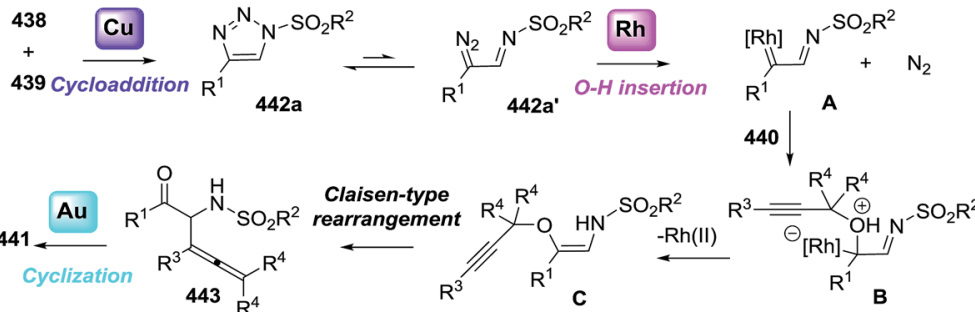

c)

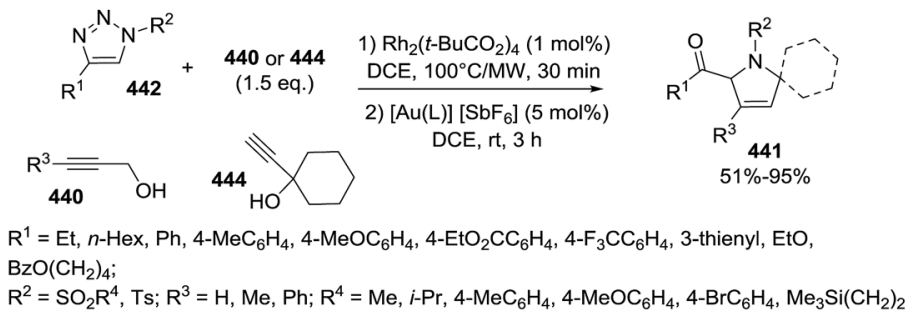

Scheme 138 Sequential one-pot synthesis of 3-pyrrolines catalyzed by three different metals ( $\mathrm{Cu}$, Rh and Au). (a) Three transformations with a single, final work-up. (b) Plausible mechanism. (c) Evaluation of the sequential independent Rh-catalyzed and Au-catalyzed reactions.

as starting materials in combination with propargylic alcohols 440 or 1-ethynylcyclohexan-1-ol 444.

\section{Conclusions}

In this review, we describe the latest advances in the transition metal-catalyzed or -mediated synthesis of the three classes of pyrrolines, illustrating the most important aspects of each synthetic method. These advances are classified according to the metal involved in the formation of the pyrroline ring. It is worth noting that a comprehensive and up-to-date compilation on the existing methodologies for the synthesis of the three classes of pyrrolines is missing in the literature. Transition metal-catalyzed reactions are no longer a niche of the organometallic chemist but have entered the mainstream synthesis of heterocycles and complex natural products. Efficiency, mild reaction conditions, and tolerance of a wide variety of functional groups are key characteristics of many of these metalmediated syntheses. There are many articles encompassing a variety of methodologies that use metals in the synthesis of the pyrroline ring. The most common metals used are copper, gold, silver, palladium, rhodium and ruthenium. A popular methodology is the palladium-catalyzed synthesis of pyrrolines from alkene-tethered oxime esters, which allows decorating the pyrroline ring with different functional groups. Another methodology that we would like to highlight is the use of $N$-sulfonyl1,2,3-triazoles as precursors of $\alpha$-imino rhodium carbene (azavinylcarbenes), which can react with a variety of olefins to produce enantioenriched pyrrolines if chiral rhodium catalysts are used. The metal-catalyzed rearrangement of vinylaziridines to pyrrolines is another exceptionally reliable methodology. Metals such as scandium and nickel have been used lately with excellent results. For example, the scandium-catalyzed synthesis of multi-substituted pyrrolines through sequential reactions has generated complex heterocycles. Nickel catalysts have the advantage that they do not suffer $\beta$ - $\mathrm{H}$-elimination in cross-coupling reactions, which contrast with the situation met with those based on palladium.

The intention of this review is to inspire further development of the metal-catalyzed synthesis of nitrogen heterocycles, using the pyrrolines as a particular example. Future developments in the transition metal-catalyzed synthesis of pyrrolines will probably involve the adoption of greener reaction conditions, using easily-accessible and less expensive metal catalysts. An increased effort should be devoted to the design of new metalcatalyzed visible-light photo-induced reactions leading to pyrrolines and other nitrogen heterocycles. Apart from being conceptually appealing, these processes are usually efficient, enantioselective and green.

The presence of the pyrroline ring in many bioactive compounds and the remarkable breadth of their reactivity justified the development of new synthetic methods for their construction. Newer approaches to the synthesis of multisubstituted pyrrolines and catalytic asymmetric methodologies for the synthesis of pyrrolines, will be required in the future 
to further develop these heterocycles for materials and for medicinal chemistry.

\section{Conflicts of interest}

There are no conflicts to declare.

\section{Acknowledgements}

The authors gratefully acknowledge Dr Jed F. Fisher, University of Notre Dame, for critical discussions of the manuscript. The research activities of this laboratory are supported by Agencia Nacional de Promoción Científica y Tecnológica (PICT-20152449 and PICT-2016-1069) and Consejo Nacional de Investigaciones Científicas y Técnicas (CONICET, Argentina).

\section{References}

1 V. C. Clark, C. J. Raxworthy, V. Rakotomalala, P. Sierwald and B. L. Fisher, Proc. Natl. Acad. Sci. U. S. A., 2005, 102, 11617.

2 K. L. Rinehart, J. Kobayashi, G. C. Harbour, J. Gilmore, M. Mascal, T. G. Holt, L. S. Shield and F. Lafargue, J. Am. Chem. Soc., 1987, 109, 3378-3387.

3 C. Marti and E. M. Carreira, J. Am. Chem. Soc., 2005, 127, 11505-11515.

4 D. Tsukamoto, M. Shibano, R. Okamoto and G. Kusano, Chem. Pharm. Bull., 2001, 49, 492-496.

5 A. Adams and N. De Kimpe, Chem. Rev., 2006, 106, 22992319.

6 T.-C. Huang, C.-S. Teng, J.-L. Chang, H.-S. Chuang, C.-T. Ho and M.-L. Wu, J. Agric. Food Chem., 2008, 56, 7399-7404.

7 C. B. Cui, H. Kakeya and H. Osada, J. Antibiot., 1996, 49, $832-835$.

8 L. H. Hurley and R. Petrusek, Nature, 1979, 282, 529-531.

9 M. Ozawa, T. Etoh, M. Hayashi, K. Komiyama, A. Kishida and A. Ohsaki, Bioorg. Med. Chem. Lett., 2009, 19, 234-236.

10 The Porphyrin Handbook, ed. K. M. Kadish, K. M. Smith and R. Guilard, Academic, San Diego, 2003.

11 S. Tyroller, W. Zwickenpflug and E. Richter, J. Agric. Food Chem., 2002, 50, 4909-4915.

12 D. Bacos, J. J. Basselier, J. P. Celerler, C. Lange, E. Marx, G. Lhommet, P. Escoubas, M. Lemaire and J. L. Clement, Tetrahedron Lett., 1988, 29, 3061-3064.

13 S. Castellano, H. D. G. Fiji, S. S. Kinderman, M. Watanabe, P. de Leon, F. Tamanoi and O. Kwon, J. Am. Chem. Soc., 2007, 129, 5843-5845.

14 S. Schann, V. Bruban, K. Pompermayer, J. Feldman, B. Pfeiffer, P. Renard, E. Scalbert, P. Bousquet and J.-D. Ehrhardt, J. Med. Chem., 2001, 44, 1588-1593.

15 J.-B. Behr, M. S. M. Pearson, C. Bello, P. Vogel and R. Plantier-Royon, Tetrahedron: Asymmetry, 2008, 19, 1829-1832.

16 I. V. Magedov, G. Luchetti, N. M. Evdokimov, M. Manpadi, W. F. A. Steelant, S. Van slambrouck, P. Tongwa, M. Y. Antipin and A. Kornienko, Bioorg. Med. Chem. Lett., 2008, 18, 1392-1396.
17 Q.-Y. Mou, J. Chen, Y.-C. Zhu, D.-H. Zhou, Z.-Q. Chi and Y.-Q. Long, Bioorg. Med. Chem. Lett., 2002, 12, 2287-2290.

18 D. Rondeau, P. Gill, M. Chan, K. Curry and W. D. Lubell, Bioorg. Med. Chem. Lett., 2000, 10, 771-773.

19 C. D. Cox, P. J. Coleman, M. J. Breslin, D. B. Whitman, R. M. Garbaccio, M. E. Fraley, C. A. Buser, E. S. Walsh, K. Hamilton, M. D. Schaber, R. B. Lobell, W. Tao, J. P. Davide, R. E. Diehl, M. T. Abrams, V. J. South, H. E. Huber, M. Torrent, T. Prueksaritanont, C. Li, D. E. Slaughter, E. Mahan, C. Fernandez-Metzler, Y. Yan, L. C. Kuo, N. E. Kohl and G. D. Hartman, J. Med. Chem., 2008, 51, 4239-4252.

20 S. Peddibhotla and J. J. Tepe, J. Am. Chem. Soc., 2004, 126, 12776-12777.

21 D. Imbri, N. Netz, M. Kucukdisli, L. M. Kammer, P. Jung, A. Kretzschmann and T. Opatz, J. Org. Chem., 2014, 79, 11750-11758.

22 X. F. Bai, L. Li, Z. Xu, Z. J. Zheng, C. G. Xia, Y. M. Cui and L. W. Xu, Chem.-Eur. J., 2016, 22, 10399-10404.

23 M. K. Majhail, P. M. Ylioja and M. C. Willis, Chem.-Eur. J., 2016, 22, 7879-7884.

24 G. Dannhardt and W. Kiefer, Arch. Pharm., 2001, 334, 183188.

25 B. B. Snider and B. J. Neubert, Org. Lett., 2005, 7, 2715-2718.

26 F. A. Davis, N. Theddu and R. Edupuganti, Org. Lett., 2010, 12, 4118-4121.

27 V. B. Reddy Iska, V. Verdolino, O. Wiest and P. Helquist, J. Org. Chem., 2010, 75, 1325-1328.

28 M. M. Nebe, M. Kucukdisli and T. Opatz, J. Org. Chem., 2016, 81, 4112-4121.

29 J. M. Humphrey, Y. Liao, A. Ali, T. Rein, Y.-L. Wong, H.-J. Chen, A. K. Courtney and S. F. Martin, J. Am. Chem. Soc., 2002, 124, 8584-8592.

30 R. Martin, A. Jager, M. Bohl, S. Richter, R. Fedorov, D. J. Manstein, H. O. Gutzeit and H. J. Knolker, Angew. Chem., Int. Ed., 2009, 48, 8042-8046.

31 J. Wegner, S. V. Ley, A. Kirschning, A.-L. Hansen, J. Montenegro Garcia and I. R. Baxendale, Org. Lett., 2012, 14, 696-699.

32 H. Zhang and D. P. Curran, J. Am. Chem. Soc., 2011, 133, 10376-10378.

33 T. Ritthiwigrom, A. C. Willis and S. G. Pyne, J. Org. Chem., 2010, 75, 815-824.

34 S. Kaden and H.-U. Reissig, Org. Lett., 2006, 8, 4763-4766. 35 V. Dhand, J. A. Draper, J. Moore and R. Britton, Org. Lett., 2013, 15, 1914-1917.

36 A. L. L. Garcia, M. J. S. Carpes, A. C. B. M. de Oca, M. A. G. dos Santos, C. C. Santana and C. R. D. Correia, J. Org. Chem., 2005, 70, 1050-1053.

37 S. G. Davies, A. M. Fletcher, I. T. T. Houlsby, P. M. Roberts and J. E. Thomson, J. Org. Chem., 2017, 82, 6689-6702.

38 F. Bellina and R. Rossi, Tetrahedron, 2006, 62, 7213-7256.

39 M. G. A. Shvekhgeimer, Chem. Heterocycl. Compd., 2003, 39, 405-448.

40 P. N. D. Singh, R. F. Klima, S. Muthukrishnan, R. S. Murthy, J. Sankaranarayanan, H. M. Stahlecker, B. Patel and A. D. Gudmundsdóttir, Tetrahedron Lett., 2005, 46, 4213-4217. 
41 S. Asghari and M. Qandalee, Synth. Commun., 2010, 40, 2172-2177.

42 E. Elamparuthi, S. Sarathkumar, S. Girija and V. Anbazhagan, Tetrahedron Lett., 2014, 55, 3992-3995.

43 A. Claesson, C. Sahlberg and K. Luthman, Acta Chem. Scand., 1979, 33, 309-310.

44 R. K. Dieter and H. Yu, Org. Lett., 2001, 3, 3855-3858.

45 R. K. Dieter, N. Chen and V. K. Gore, J. Org. Chem., 2006, 71, 8755-8760.

46 S. Mangelinckx, N. Giubellina and N. De Kimpe, Chem. Rev., 2004, 104, 2353-2400.

47 B. Cui, J. Ren and Z. W. Wang, J. Org. Chem., 2014, 79, 790796.

48 P. Pandit, N. Chatterjee and D. K. Maiti, Chem. Commun., 2011, 47, 1285-1287.

49 P. A. Wender and D. Strand, J. Am. Chem. Soc., 2009, 131, 7528-7529.

50 Y.-Q. Fang and E. N. Jacobsen, J. Am. Chem. Soc., 2008, 130, 5660-5661.

51 C. E. Henry, Q. Xu, Y. C. Fan, T. J. Martin, L. Belding, T. Dudding and O. Kwon, J. Am. Chem. Soc., 2014, 136, 11890-11893.

52 P. J. Campos, A. Soldevilla, D. Sampedro and M. A. Rodríguez, Tetrahedron Lett., 2002, 43, 8811-8813.

53 P. J. Campos, A. Soldevilla, D. Sampedro and M. A. Rodríguez, Org. Lett., 2001, 3, 4087-4089.

54 A. Soldevilla, D. Sampedro, P. J. Campos and M. A. Rodríguez, J. Org. Chem., 2005, 70, 6976-6979.

55 R. S. Atkinson and C. W. Rees, Chem. Commun., 1967, 1232.

56 T. Hudlicky and J. W. Reed, Angew. Chem., Int. Ed., 2010, 49, 4864-4876.

57 Y. J. Liang, D. W. Dong, Y. M. Lu, Y. Wang, W. Pan, Y. Y. Chai and Q. Liu, Synthesis, 2006, 3301-3304.

58 G. Sathishkannan and K. Srinivasan, Org. Lett., 2011, 13, 6002-6005.

59 D. H. Zhang, Z. Zhang and M. Shi, Chem. Commun., 2012, 48, 10271-10279.

60 R. Miyauchi, C. Ono, T. Ohnuki and Y. Shibad, Appl. Environ. Microbiol., 2016, 82, 6414-6422.

61 E. Tsujii, M. Muroi, N. Shiragami and A. Takatsuki, Biochem. Biophys. Res. Commun., 1996, 220, 459-466.

62 M. X. Zhao, H. K. Zhu, T. L. Dai and M. Shi, J. Org. Chem., 2015, 80, 11330-11338.

63 T. J. Hagen, A. A. Bergmanis, S. W. Kramer, K. F. Fok, A. E. Schmelzer, B. S. Pitzele, L. Swenton, G. M. Jerome, C. M. Kornmeier, W. M. Moore, L. F. Branson, J. R. Connor, P. T. Manning, M. G. Currie and E. A. Hallinan, J. Med. Chem., 1998, 41, 3675-3683.

64 V. Vaillancourt and S. M. K. Sheehan, PCT Int. Appl. WO2013169622A1, 2013.

65 D. Aicher, A. Wiehe, C. B. W. Stark, and V. Albrechr, US Pat., Appl. US20130041307A1, 2013.

66 D. Aicher, V. Albrecht, B. Gitter, C. B. W. Stark, and A. Wiehe, PCT Int. Appl. WO2013015774A1, 2013.

67 D. Aicher, S. Gräfe, C. B. W. Stark and A. Wiehe, Bioorg. Med. Chem. Lett., 2011, 21, 5808-5811.
68 D. Aicher, A. Wiehe, C. B. W. Stark, V. Albrecht and S. Grafe, PCT Int. Appl. WO2012012809A2, 2012.

69 R. M. Rosser and D. J. Faulkner, J. Org. Chem., 1984, 49, 5157-5160.

70 E. T. Newcomb, P. C. Knutson, B. A. Pedersen and E. M. Ferreira, J. Am. Chem. Soc., 2016, 138, 108-111.

71 S. Sanjaya, S. H. Chua and S. Chiba, Synlett, 2012, 16571661.

72 W. Debrouwer, T. S. A. Heugebaert, K. Van Hecke and C. V. Stevens, J. Org. Chem., 2013, 78, 8232-8241.

73 X.-H. Ouyang, R.-J. Song, Y. Liu, M. Hu and J.-H. Li, Org. Lett., 2015, 17, 6038-6041.

74 J. Duan, Y. Cheng, R. Li and P. Li, Org. Chem. Front., 2016, 3, 1614-1618.

75 S.-P. Jiang, Y.-T. Su, K.-Q. Liu, Q.-H. Wu and G.-W. Wang, Chem. Commun., 2015, 51, 6548-6551.

76 B. Liu, Z.-M. Zhang, B. Xu, S. Xu, H.-H. Wu, Y. Liu and J. Zhang, Org. Chem. Front., 2017, 4, 1772-1776.

77 A. Faulkner, N. J. Race, J. S. Scott and J. F. Bower, Chem. Sci., 2014, 5, 2416-2421.

78 T. Saegusa, Y. Ito, H. Kinoshita and S. Tomita, J. Org. Chem., 1971, 36, 3316-3323.

79 S. Padilla, J. Adrio and J. C. Carretero, J. Org. Chem., 2012, 77, 4161-4166.

80 K. Goutham, N. Mangina, S. Suresh, P. Raghavaiah and G. V. Karunakar, Org. Biomol. Chem., 2014, 12, 2869-2873.

81 N. S. Medran, M. Villalba, E. G. Mata and S. A. Testero, Eur. J. Org. Chem., 2016, 3757-3764.

82 A. D. Melhado, G. W. Amarante, Z. J. Wang, M. Luparia and F. D. Toste, J. Am. Chem. Soc., 2011, 133, 3517-3527.

83 K. Guo, H. Zhang, S. Cao, C. Gu, H. Zhou, J. Li and Y. Zhu, Org. Lett., 2018, 20, 2261-2264.

84 S.-H. Cai, J. H. Xie, S. J. Song, L. Ye, C. Feng and T. P. Loh, ACS Catal., 2016, 6, 5571-5574.

85 S.-H. Cai, D.-X. Wang, L. Ye, Z.-Y. Liu, C. Feng and T.-P. Loh, Adv. Synth. Catal., 2018, 360, 1262-1266.

86 H. Jiang and A. Studer, Angew. Chem., Int. Ed., 2017, 56, 12273-12276.

87 C. Hua, K. Q. Vuong, M. Bhadbhade and B. A. Messerle, Organometallics, 2012, 31, 1790-1800.

88 H. Kawai, T. Kitayama, E. Tokunaga, T. Matsumoto, H. Sato, M. Shiro and N. Shibata, Chem. Commun., 2012, 48, 40674069.

89 H. Kawai, Z. Yuan, T. Kitayama, E. Tokunaga and N. Shibata, Angew. Chem., Int. Ed., 2013, 52, 5575-5579.

90 H. B. Yang and N. Selander, Chem.-Eur. J., 2017, 23, 17791783.

91 M. M. Jackman, Y. Cai and S. L. Castle, Synthesis, 2017, 49, 1785-1795.

92 T. Shimbayashi, K. Okamoto and K. Ohe, Chem.-Asian J., 2018, 13, 395-399.

93 M. Bingham, C. Moutrille and S. Z. Zard, Heterocycles, 2014, 88, 953-960.

94 H. B. Yang, S. R. Pathipati and N. Selander, ACS Catal., 2017, 7, 8441-8445.

95 L. Wang and C. Wang, Org. Chem. Front., 2018, 5, 34763482. 
96 A. Faulkner, J. S. Scott and J. F. Bower, J. Am. Chem. Soc., 2015, 137, 7224-7230.

97 A. Faulkner, J. S. Scott and J. F. Bower, Chem. Commun., 2013, 49, 1521-1523.

98 N. J. Race and J. F. Bower, Org. Lett., 2013, 15, 4616-4619.

99 N. J. Race, A. Faulkner, G. Fumagalli, T. Yamauchi, J. S. Scott, M. Ryden-Landergren, H. A. Sparkes and J. F. Bower, Chem. Sci., 2017, 8, 1981-1985.

100 X. Bao, Q. Wang and J. Zhu, Angew. Chem., Int. Ed., 2017, 56, 9577-9581.

101 Z. Shi, M. Suri and F. Glorius, Angew. Chem., Int. Ed., 2013, 52, 4892-4896.

102 C. Chen, L. Hou, M. Cheng, J. Su and X. Tong, Angew. Chem., Int. Ed., 2015, 54, 3092-3096.

103 D.-Y. Li, S. Liu, S. Chen, A. Wang, X.-P. Zhu and P.-N. Liu, ACS Catal., 2018, 8, 6407-6412.

104 F. Alonso, I. P. Beletskaya and M. Yus, Chem. Rev., 2004, 104, 3079-3160.

105 T. E. Müller, K. C. Hultzsch, M. Yus, F. Foubelo and M. Tada, Chem. Rev., 2008, 108, 3795-3892.

106 X. Huang, X. Li, X. Xie, K. Harms, R. Riedel and E. Meggers, Nat. Commun., 2017, 8, 2245.

107 V. Kanchupalli and S. Katukojvala, Angew. Chem., Int. Ed., 2018, 57, 5433-5437.

108 D. Chao, T.-X. Liu, N. Ma, P. Zhang, Z. Fu, J. Ma, Q. Liu, F. Zhang, Z. Zhang and G. Zhang, Chem. Commun., 2016, 52, 982-985.

109 Z.-P. Wang, S. Xiang, P.-L. Shao and Y. He, J. Org. Chem., 2018, 83, 10995-11007.

110 A. Mondal and C. Mukhopadhyay, Eur. J. Org. Chem., 2017, 6299-6313.

111 J. J. Badillo, C. J. A. Ribeiro, M. M. Olmstead and A. K. Franz, Org. Lett., 2014, 16, 6270-6273.

112 X. Zhu and S. Chiba, Chem. Commun., 2016, 52, 2473-2476.

113 J. N. Zhu, L. L. Chen, R. X. Zhou, B. Li, Z. Y. Shao and S. Y. Zhao, Org. Lett., 2017, 19, 6044-6047.

114 K. Liu, C. Zhu, J. Min, S. Peng, G. Xu and J. Sun, Angew. Chem., Int. Ed., 2015, 54, 12962-12967.

115 M. Yoshida, A. Kobayashi, A. Nakayama and K. Namba, Tetrahedron, 2016, 72, 2544-2551.

116 R. Wang, Y. OuYang, C. Xu, N. Yi, J. Jiang, W. Deng, Z. Zeng and J. Xiang, Org. Biomol. Chem., 2017, 15, 796-800.

117 S.-P. Jiang, Q.-H. Wu and G.-W. Wang, J. Org. Chem., 2017, 82, 10823-10829.

118 B. Xu, Z.-M. Zhang, L. Zhou and J. Zhang, Org. Lett., 2018, 20, 2716-2719.

119 X. Shi, X. Chen, M. Wang, X. Zhang and X. Fan, J. Org. Chem., 2018, 83, 6524-6533.

120 B. Ritzen, G. J. J. Richelle, L. Brocken, F. L. van Delft and F. Rutjes, Synlett, 2014, 25, 270-274.

121 D. Monge, K. L. Jensen, P. T. Franke, L. Lykke and K. A. Jørgensen, Chem.-Eur. J., 2010, 16, 9478-9484.

122 Y. F. Yu, C. Shu, B. Zhou, J. Q. Li, J. M. Zhou and L. W. Ye, Chem. Commun., 2015, 51, 2126-2129.

123 Z.-S. Wang, T.-D. Tan, C.-M. Wang, D.-Q. Yuan, T. Zhang, P. Zhu, C. Zhu, J.-M. Zhou and L.-W. Ye, Chem. Commun., 2017, 53, 6848-6851.
124 L. M. Joyce, A. C. Willis, C. J. T. Hyland and S. G. Pyne, Aust. J. Chem., 2018, 71, 682-689.

125 M. C. Chung, Y. H. Chan, W. J. Chang and D. R. Hou, Org. Biomol. Chem., 2017, 15, 3783-3790.

126 C. R. Liu, B. H. Zhu, J. C. Zheng, X. L. Sun, Z. W. Xie and Y. Tang, Chem. Commun., 2011, 47, 1342-1344.

127 B. Sun, Q. Ma, Y. Wang, Y. Zhao, P. Liao and X. Bi, Eur. J. Org. Chem., 2014, 2014, 7552-7555.

128 O. El-Sepelgy, A. Brzozowska, J. Sklyaruk, Y. K. Jang, V. Zubar and M. Rueping, Org. Lett., 2018, 20, 696-699.

129 T. Y. Lin, H. H. Wu, J. J. Feng and J. L. Zhang, Org. Lett., 2017, 19, 6526-6529.

130 J.-C. Zeng, H. Xu, R.-L. Huang, F. Yu and Z. Zheng, Tetrahedron Lett., 2018, 59, 1576-1580.

131 M. C. Martin, D. V. Patil and S. France, J. Org. Chem., 2014, 79, 3030-3039.

132 W. Zhou, G. An, G. Zhang, J. Han and Y. Pan, Org. Biomol. Chem., 2011, 9, 5833-5837.

133 J. Peng, Y. Zhao, J. Zhou, Y. Ding and C. Chen, Synthesis, 2014, 46, 2051-2056.

134 B. Jiang, F. F. Meng, Q. J. Liang, Y. H. Xu and T. P. Loh, Org. Lett., 2017, 19, 914-917.

135 A. K. A. Persson and J.-E. Bäckvall, Angew. Chem., Int. Ed., 2010, 49, 4624-4627.

136 K. Okamoto, T. Oda, S. Kohigashi and K. Ohe, Angew. Chem., Int. Ed., 2011, 50, 11470-11473.

137 M. Yoshida, K. Kinoshita and K. Namba, Org. Biomol. Chem., 2014, 12, 2394-2403.

138 P. V. Santhini, G. Nimisha, J. John, E. Suresh, R. L. Varma and K. V. Radhakrishnan, Chem. Commun., 2017, 53, 1848-1851.

139 T. Zheng, D.-S. Shan, B. Jin and R.-F. Peng, Org. Biomol. Chem., 2018, 16, 8845-8853.

140 Y. Y. Zhang, Y. Wei, X. Y. Tang and M. Shi, Chem. Commun., 2017, 53, 5966-5969.

141 H. Kusama, Y. Karibe, R. Imai, Y. Onizawa, H. Yamabe and N. Iwasawa, Chem.-Eur. J., 2011, 17, 4839-4848.

142 S. Kim, J. Mo, J. Kim, T. Ryu and P. H. Lee, Asian J. Org. Chem., 2014, 3, 926-931.

143 T. Miura, T. Tanaka, K. Hiraga, S. G. Stewart and M. Murakami, J. Am. Chem. Soc., 2013, 135, 13652-13655.

144 S. W. Kwok, L. Zhang, N. P. Grimster and V. V. Fokin, Angew. Chem., Int. Ed., 2014, 53, 3452-3456.

145 X. Ma, L. Liu, J. Wang, X. Xi, X. Xie and H. Wang, J. Org. Chem., 2018, 83, 14518-14526.

146 H. Shang, Y. H. Wang, Y. Tian, J. Feng and Y. F. Tang, Angew. Chem., Int. Ed., 2014, 53, 5662-5666.

147 X. Zheng, B. Cao and X. Zhang, Tetrahedron Lett., 2014, 55, 4489-4491.

148 J.-J. Feng, T.-Y. Lin, C.-Z. Zhu, H. Wang, H.-H. Wu and J. Zhang, J. Am. Chem. Soc., 2016, 138, 2178-2181.

149 C. E. Kim, Y. Park, S. Park and P. H. Lee, Adv. Synth. Catal., 2015, 357, 210-220.

150 B. Alcaide, P. Almendros, S. Cembellin, T. M. del Campo and G. Palop, Chem.-Eur. J., 2017, 23, 13754-13759.

151 M. K. Ghorai and D. P. Tiwari, J. Org. Chem., 2013, 78, 26172625. 
152 Y. Xia, X. Liu, H. Zheng, L. Lin and X. Feng, Angew. Chem., Int. Ed., 2015, 54, 227-230.

153 M. Schlegel, P. Coburger and C. Schneider, Chem.-Eur. J., 2018, 24, 14207-14212.

154 C. Arroniz, A. Gil-Gonzalez, V. Semak, C. Escolano, J. Bosch and M. Amat, Eur. J. Org. Chem., 2011, 3755-3760.

155 J. Song, C. Guo, P.-H. Chen, J. Yu, S.-W. Luo and L.-Z. Gong, Chem.-Eur. J., 2011, 17, 7786-7790.

156 W. K. Anderson and A. S. Milowsky, J. Med. Chem., 1987, 30, 2144-2147.

157 G. Bhaskar, Y. Arun, C. Balachandran, C. Saikumar and P. T. Perumal, Eur. J. Med. Chem., 2012, 51, 79-91.

158 W. Shi, Y. Duan, Y. Qian, M. Li, L. Yang and W. Hu, Bioorg. Med. Chem. Lett., 2010, 20, 3592-3595.

159 M. Brichacek, M. Navarro Villalobos, A. Plichta and J. T. Njardarson, Org. Lett., 2011, 13, 1110-1113.

160 Q. Wu, J. Hu, X. Ren and J. Zhou, Chem.-Eur. J., 2011, 17, 11553-11558.

161 M. Sai, H. Yorimitsu and K. Oshima, Angew. Chem., Int. Ed., 2011, 50, 3294-3298.

162 W. Rao, P. Kothandaraman, C. B. Koh and P. W. H. Chan, Adv. Synth. Catal., 2010, 352, 2521-2530.

163 C. Zheng, Y. Wang and R. Fan, Org. Lett., 2015, 17, 916-919.

164 F. F. Tang, W. L. Yang, X. X. Yu and W. P. Deng, Catal. Sci. Technol., 2015, 5, 3568-3575.

165 E. J. Groso, A. N. Golonka, R. A. Harding, B. W. Alexander, T. M. Sodano and C. S. Schindler, ACS Catal., 2018, 20062011.

166 S. R. K. Minkler, B. H. Lipshutz and N. Krause, Angew. Chem., Int. Ed., 2011, 50, 7820-7823.

167 G. M. O. Amombo, O. Floge, S. K. D. Kalai, S. Schoder, U. Warzok and H. U. Reissig, Eur. J. Org. Chem., 2017, 1965-1972.

168 M. O. Amombo, A. Hausherr and H.-U. Reissig, Synlett, 1999, 1999, 1871-1874.

169 T. Miura, T. Tanaka, K. Matsumoto and M. Murakami, Chem.-Eur. J., 2014, 20, 16078-16082.

170 D.-H. Zhang, L.-F. Yao, Y. Wei and M. Shi, Angew. Chem., Int. Ed., 2011, 50, 2583-2587.

171 Z. Wang, H. Zheng, J. Yang, X. Xie and X. She, Adv. Synth. Catal., 2015, 357, 2082-2088.

172 N. Kern, A. Blanc, J. M. Weibel and P. Pale, Chem. Commun., 2011, 47, 6665-6667.

173 M. Fuji, J. Chiwata, M. Ozaki, S. Aratani and Y. Obora, ACS Omega, 2018, 3, 8865-8873.

174 E. Leemans, F. Colpaert, S. Mangelinckx, S. De Brabandere, B. Denolf and N. De Kimpe, Synlett, 2011, 674-678.
$175 \mathrm{P} . \mathrm{Wu}, \mathrm{H} . \mathrm{Liu}$ and X. Tong, Tetrahedron Lett., 2012, 53, 4673-4675.

176 W. Sun, G. Zhu, L. Hong and R. Wang, Chem.-Eur. J., 2011, 17, 13958-13962.

177 M.-J. Zhong, H.-T. Zhu, P. Gao, Y.-F. Qiu and Y.-M. Liang, RSC Adv., 2014, 4, 8914-8917.

178 C. B. Reddy, R. Bharti, S. Kumar and P. Das, RSC Adv., 2016, 6, 71117-71121.

179 T. Rajasekaran, G. Karthik, B. Sridhar and B. V. S. Reddy, Org. Lett., 2013, 15, 1512-1515.

180 A. Deiters and S. F. Martin, Chem. Rev., 2004, 104, 21992238.

181 A. Perfetto, C. Costabile, P. Longo, V. Bertolasi and F. Grisi, Chem.-Eur. J., 2013, 19, 10492-10496.

182 Q. Yang, H. Alper and W.-J. Xiao, Org. Lett., 2007, 9, 769771.

183 K. M. Kuhn, T. M. Champagne, S. H. Hong, W.-H. Wei, A. Nickel, C. W. Lee, S. C. Virgil, R. H. Grubbs and R. L. Pederson, Org. Lett., 2010, 12, 984-987.

184 S. Sawadjoon and J. S. M. Samec, Org. Biomol. Chem., 2011, 9, 2548-2554.

185 A. Bunrit, S. Sawadjoon, S. Tsupova, P. J. R. Sjoberg and J. S. M. Samec, J. Org. Chem., 2016, 81, 1450-1460.

186 M. B. Tait, S. Butterworth and J. Clayden, Org. Lett., 2015, 17, 1236-1239.

187 W. Sun, X. Ma, L. Hong and R. Wang, J. Org. Chem., 2011, 76, 7826-7833.

188 S. Ishikawa, F. Noguchi and A. Kamimura, J. Org. Chem., 2010, 75, 3578-3586.

189 Y. Nagao, W.-M. Dai, M. Ochiai and M. Shiro, Tetrahedron, 1990, 46, 6361-6380.

190 K. Csatayova, S. G. Davies, A. L. A. Figuccia, A. M. Fletcher, J. G. Ford, J. A. Lee, P. M. Roberts, B. G. Saward, H. Song and J. E. Thomson, Tetrahedron, 2015, 71, 9131-9142.

191 L. Li and J. Zhang, Org. Lett., 2011, 13, 5940-5943.

192 R. K. Dieter, N. Chen, H. Yu, L. E. Nice and V. K. Gore, J. Org. Chem., 2005, 70, 2109-2119.

193 B. Mitasev and K. M. Brummond, Synlett, 2006, 2006, 31003104.

194 M. Sai and S. Matsubara, Org. Lett., 2011, 13, 4676-4679.

195 J. Brioche, C. Meyer and J. Cossy, Org. Lett., 2013, 15, 16261629.

196 R. R. Tata, C. Fu, S. P. Kelley and M. Harmata, Org. Lett., 2018, 20, 5723-5726.

197 S. S. K. Boominathan, W. P. Hu, G. C. Senadi and J. J. Wang, Adv. Synth. Catal., 2013, 355, 3570-3574.

198 A. Hausherr and H.-U. Reissig, Eur. J. Org. Chem., 2018, 2018, 4071-4080. 